\title{
Defects on Carbons for Electrocatalytic Oxygen Reduction
}

Xuecheng Yan, Yi Jia, Xiangdong Yao*

Queensland Micro- and Nanotechnology Centre, Griffith University, Nathan Campus, QLD 4111, Australia

*E-mail: x.yao@griffith.edu.au 


\begin{abstract}
The exploration of highly active and durable cathodic oxygen reduction reaction (ORR) catalysts with economical production cost is still the bottleneck to realize the large-scale commercialization of fuel cells. In recent years, remarkable progresses have been achieved in fabricating effective nonprecious metal based ORR catalysts. Particularly, modified carbon materials have aroused extensive research interest because of their excellent performance and low cost. In this review, we present an overview on recent advancement in developing defective carbon based materials for catalyzing the ORR. Specifically, three general kinds of defective carbons electrocatalysts will be summarized. They are nonmetal induced defective carbons (modified by heteroatoms), intrinsic defective carbons (defects created by a physical or chemical method), and atomic metal species induced/coordinated defective carbons (metal-macrocycle complexes with different coordination environments). The common configurations of various defective carbons will be discussed, with typical examples on recently developed both metalfree and precious/non-precious metal species coordinated carbons. At last, the future research directions of the defective carbon materials are proposed. The newly established defects promoted catalysis mechanism will be beneficial for the design and fabrication highly effective electrocatalysts for practical energy storage and conversion applications.
\end{abstract}




\section{Introduction}

The explosive growth of the world population and the rapid development of modern economy have resulted in a series of problems, such as the deterioration of the ecological environment and the dramatic reduction of fossil-based fuels. In order to tackle these serious issues that threaten and slow down the advancement of human beings, the exploration and utilization of clean energy is of pivotal importance. Typical examples include the promotion and popularization of the advocated "Hydrogen Economy", and developing technologies to store and utilize clean energy. Particularly, hydrogen fuel cells have been intensively investigated because of their guaranteed safety, high-energy conversion efficiency as well as pollution-free features. It is believed that fuel cell is one of the cleanest technologies to be used in the future portable electronic products and transportation systems. However, one of the major drawbacks of fuel cells is the sluggish cathodic oxygen reduction reaction (ORR) that greatly restricts the overall cell performance. Normally, a catalyst is used to accelerate the ORR. Unfortunately, the expensive platinum $(\mathrm{Pt})$ is still the current most active ORR electrocatalyst, particularly in acidic media. ${ }^{1-4}$ In recent years, extensive research work has been devoted to exploiting cost-effective and efficient alternatives to replace the commercial Pt-based ORR catalysts, including: (i) Downsize the Pt particles to maximize the atomic efficiency or alloy $\mathrm{Pt}$ with non-precious metals, such as $\mathrm{Fe}, \mathrm{Co}, \mathrm{Ni}$ and $\mathrm{Cu}$ to reduce the utilization of Pt; ;-9 $^{5 i i)}$ Explore non-precious metal-based ORR catalysts to completely replace Pt-based counterparts, ${ }^{10-15}$ and (iii) Develop metal-free electrocatalysts. ${ }^{16-19}$ Comparatively, metal-free based carbon materials are ideal as the cathodic ORR catalysts for practical fuel cell applications from a long-term perspective. Primarily because it is not a durable and sustainable way of using Pt-containing materials as the ORR catalysts due to the scarcity of Pt resources. Besides, transition metal-based materials are subject to dissolve and aggregate under fuel cell operating conditions. This will make them unsuitable for practical applications because both their activity and durability would suffer severe losses..$^{20,21}$

The distinct natures of carbon materials, such as good electrical conductivity, high surface area, excellent thermal stability, and outstanding chemical stability in non-oxidizing atmospheres render them appropriate for a wide range of applications, ${ }^{22-29}$ a case in point is as the ORR catalyst. During the past decade, tremendous research work has been concentrated on developing modified carbon materials for catalyzing the ORR, since the unadulterated carbons are not active for electrocatalysis. One approach is to incorporate various heteroatoms, such as nitrogen $(\mathrm{N})$, phosphorus $(\mathrm{P})$, sulphur $(\mathrm{S})$, boron $(\mathrm{B})$ and fluorine $(\mathrm{F})$, into the carbon materials. This was marked by the investigation on synthesizing nitrogendoped carbon nanotube arrays for efficient catalyzing the ORR in Dai's group in 2009. ${ }^{16}$ The other effective method is to create topological defects in carbon materials to enhance their ORR performance. Recently published papers have been reviewed heteroatoms doped carbon materials for the ORR, ${ }^{30-32}$ or non-precious metal based ORR catalysts ${ }^{33-35}$ from the aspect of design, synthesis and characterization. However, systematic and comprehensive reviews on carbons catalyzed ORR from the angle of defect 
engineering have not been reported. As an emerging field, it is indispensable to review the latest work on defects promoted ORR, which will provide timely guidelines for the further development of this thriving area.

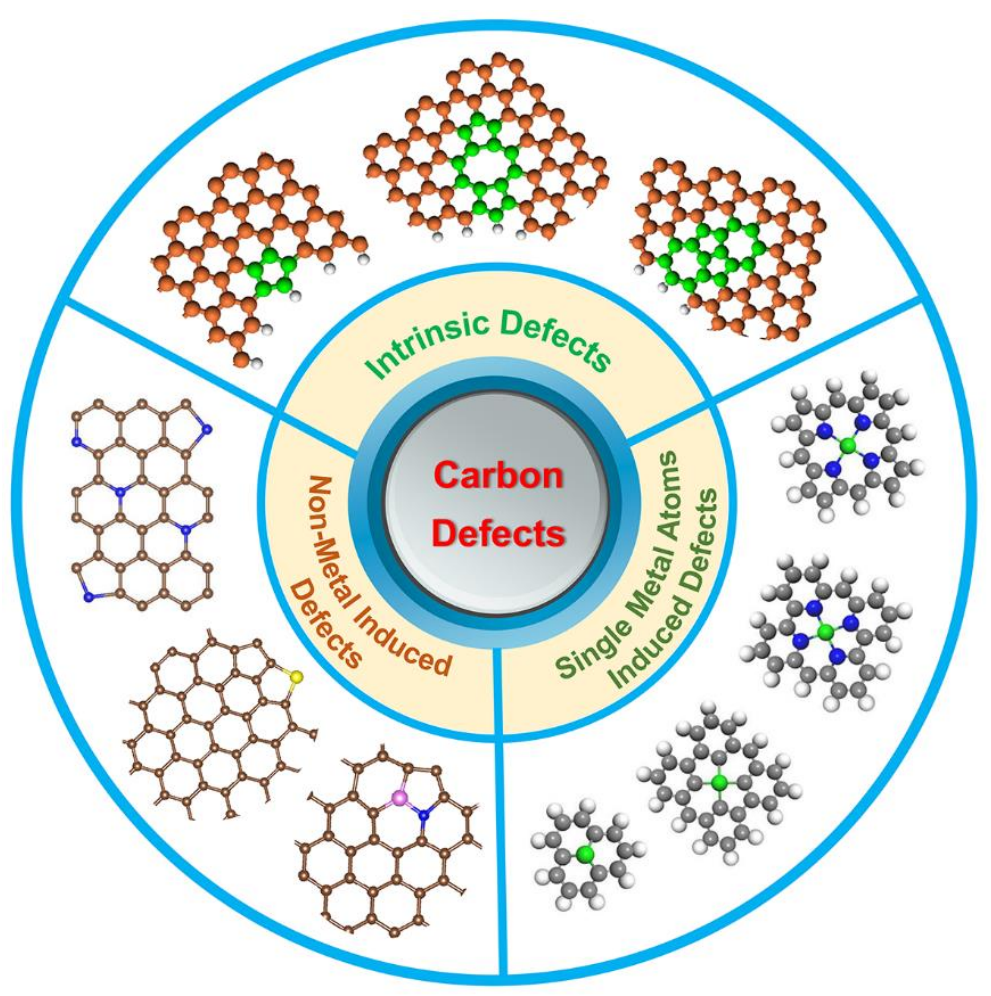

Fig. 1 Schematic illustration of three general kinds of carbon defects. ${ }^{36,37}$ Schemes of the intrinsic defect: Reprinted with permission from ref. 36. Copyright 2016, John Wiley and Sons; schemes of the single metal atoms induced defects: Reprinted with permission from ref. 37. Copyright 2018, Springer Nature.

In this review, three general kinds of defective carbons for efficient catalyzing the ORR will be summarized. They are non-metal induced defective carbons (such as N, S, B, P, F altered carbon materials), intrinsic defective carbons (such as various defects created by the removal of heteroatoms, C5, G585 and G7557), ${ }^{36}$ and atomic metal species induced/coordinated defective carbons (metalmacrocycle complexes with different coordination environments). ${ }^{37}$ The representative configurations of the three kinds of defective carbons are shown in Fig. 1. Generally, heteroatoms modification is an alternative way of introducing defects into carbon materials, as the electronic structures of the bulk carbon materials are interrupted after the incorporation of foreign elements. Therefore, heteroatoms doped carbon materials are defective carbons as well. Herein, typical heteroatoms modified carbon materials, such as carbon nanotubes, graphene and mesoporous carbons doped/co-doped with $\mathrm{N}, \mathrm{S}, \mathrm{B}$, P, F or halogenated with enhanced ORR performance will be presented briefly. It will mainly focus on uncovering the intrinsic relationships between the performance and their structures from the aspect of 
defect engineering. Particularly, recently reported defective carbon based ORR catalysts derived from different precursors by using different defect creation methods will be discussed in details, both theoretically and experimentally. At last, as a promising frontier, the newly developed atomic metal species coordinated defective carbons for electrocatalysis will be presented as well. For carbon materials coordinated with various atomic metal species, including transition metals ( $\mathrm{Fe}, \mathrm{Co}$ and $\mathrm{Ni})^{28,38-40}$ and precious metals $(\mathrm{Pt}, \mathrm{Au}),{ }^{41-43}$ it can also be categorized into defective carbons for the following reasons: (i) The total metal atoms incorporated into carbon materials are relatively low, normally below 1.0 at.\% (or 5.0 wt.\%). Therefore, the majority of the catalyst component is still carbon; (ii) Similar to the introduction of non-metal heteroatoms into the carbon matrix, atomic metal species can also be regarded as heteroatoms. They are capable of tuning the electronic structures and configurations of the carbon materials, thus producing defective carbons with improved electrocatalytic performance; ${ }^{44}$ (iii) The metal species themselves may not be the actual active sites and need to be coordinated with the surrounding non-metal atoms; and (iv) The local coordination environment plays a key role for the catalysis. ${ }^{40,45}$ Accordingly, recently reported carbon materials, particularly defective carbons captured single metal atoms, such as $\mathrm{Fe}, \mathrm{Co}$, Ni and $\mathrm{Pt}$ will be presented as well. The discussions will mainly on how the single metal atoms coordinate with the carbon/non-metal heteroatoms. This review will provide an overall and prompt update on the newly developed defective carbon based ORR electrocatalysts. It will reveal the intrinsic relationships between the structures/configurations of the defective carbons and the electrocatalysis performance, which will shed some light on the advancement of this prosperous area.

\section{Basic Concepts of Fuel Cells and ORR Mechanisms}

\subsection{Fuel Cell Description}

A fuel cell is an electrochemical device that converts chemical energy from a fuel into electricity and thermal energy through chemical reactions. Theoretically, a fuel cell will produce electricity as long as it has a fuel supply. Fuel cells are expected to play a remarkable role in decreasing the dependency on traditional fossil fuels because of their multiple advantages. For example, the high energy conversion efficiency, low environmental impact, fuel flexibility, rapid load response and modular design. All these merits render them to be one of the most promising sources of distributed energy. ${ }^{46}$ Based on the electrolyte they are using, fuel cells can be divided into different types, such as alkaline fuel cells (AFCs), proton exchange membrane fuel cells (PEMFCs), phosphoric acid fuel cells (PAFCs), molten carbonate fuel cells (MCFCs) and solid oxide fuel cells (SOFCs). ${ }^{47}$ Of which, PEMFC has attracted the most research attention because of its wide applications. For instance, it can be used in fuel cell vehicles, stationary power supply as well as portable devices. The PEMFC is considered as a potential replacement for the internal combustion engine in transportation applications. ${ }^{48-50}$ For the PEM fuel cells, catalysts play key roles in accelerating the electrode reactions, particularly for the cathodic oxygen 
reduction reaction (ORR). This is because the ORR is kinetically quite sluggish due to the $\mathrm{O}=\mathrm{O}$ bond is strong and needs high energy input to break. Besides, it also requires four coupled electron and proton transfer during the reaction process. ${ }^{51}$ It is estimated that the catalyst accounts for over $55 \%$ of the cell cost since the expensive platinum is still an essential component. ${ }^{52}$ In the past decades, tremendous studies have been carried out to develop inexpensive ORR catalysts with high performance, and great achievements have been reached, both experimentally and theoretically. ${ }^{53-55}$ In this review, only modified carbon based ORR catalyst will be summarized owing to their high activity and low cost.

\subsection{Reaction Mechanisms of the ORR}

The specific reaction pathway is complicated because the ORR is a three-phase reaction. Generally,

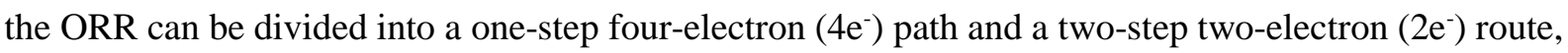
regardless the type of the catalyst and electrolyte. ${ }^{56}$ Apparently, a higher selectivity toward the $4 \mathrm{e}^{-}$ pathway is more efficient to catalyze the ORR. For modified carbon based ORR catalysts, the nature of the active site is being uncovered since remarkable effort has been devoted in the past years. Previously, the origin of the ORR activity in heteroatoms modified carbon materials was controversial. For example, some researchers stated that the introduced heteroatoms were the ORR active sites in the doped carbon structures. ${ }^{57-59}$ However, others claimed that the incorporated foreign elements were possibly not the active center, but served as a channel to create special carbon structures, such as defects, which may the actual ORR active site. ${ }^{60}$ Recent research on defects promoted ORR is a strong support to the second mechanism. ${ }^{36,61-67}$ The combination of recent theoretical and experimental investigations on defects promoted electrocatalysis reveal that the modified carbon atoms themselves are more likely the catalytic active sites. Gradually, a catalysis mechanism based on defect structures is established to explain the origin of the ORR activity in various carbon based materials. In the following sections, we will give specific examples on different defective carbon materials promoted ORR.

\section{Non-Metal Induced Defective Carbons for the ORR}

In recent years, the newly developed carbon materials, including carbon nanotubes, graphene and mesoporous carbons have attracted intensive investigations. The unique physical and chemical properties of these carbons render them as potential metal-free ORR catalysts, particularly after the modification of heteroatoms (such as N, S, B, P and F). Of which, nitrogen has been more intensively investigated than other foreign elements. Comparted to other heteroatoms, nitrogen could more effectively alter the electronic and crystal structures of the carbons, enhance their chemical stability, surface polarity, electric conductivity, and electron-donor properties. ${ }^{68-70}$ Therefore, it could effectively promote the ORR. ${ }^{16,58,71,72}$ Normally, four types of nitrogen states are considered to be relevant to the ORR. They are pyridinic N, pyrrolic N, quaternary $\mathrm{N}$ and pyridine-N-oxide. Pyridinic $\mathrm{N}$ is the nitrogen atoms located at the edge of the graphene planes, where each $\mathrm{N}$ atom coordinates with two carbon atoms 
and donates one p-electron to the aromatic $\pi$-system. ${ }^{73,74}$ Pyrrolic $\mathrm{N}$ refers to the nitrogen atoms that are incorporated into the five-membered heterocyclic rings, which are bonded with two carbon atoms and offer two p-electrons to the $\pi$-system. ${ }^{75}$ Quaternary $\mathrm{N}$, also known as graphitic $\mathrm{N}$, in which nitrogen atoms are merged into the graphene plane and bonded to three carbon atoms. ${ }^{73}$ Pyridine-N-oxide can be described as the $\mathrm{N}$ atoms that are bonded to two carbon atoms and one oxygen atom. ${ }^{75}$

Besides the configurations of the heteroatoms, it is revealed that their electronegativity may also influence the charge density of the carbon atoms in the $s p^{2}$ lattice. No matter the heteroatoms are electron-rich nitrogen and oxygen, or electron-deficient boron (the electronegativities of $\mathrm{C}, \mathrm{N}, \mathrm{O}$ and $\mathrm{B}$ atoms are 2.55, 3.04, 3.44 and 2.04, respectively). ${ }^{16,76,77}$ The modified carbon atoms may facilitate the formation of adsorption sites for oxygen and thus being beneficial for the ORR. In this section, an overview of recent development on three kinds of carbon-based ORR catalysts will be presented. They are heteroatoms modified carbon nanotubes, graphene and mesoporous carbons, since the unadulterated carbon materials normally show very low electrocatalytic activity. Particularly, the influence of the carbon microstructure and configuration, the type of the heteroatoms on the ORR performance of the synthesized defective catalysts will be discussed.

\subsection{Carbon Nanotube-Based Materials for the ORR}

Carbon nanotubes (CNTs) were firstly discovered by Iijima in $1991 .{ }^{78}$ They are tubular cylinders of carbon atoms that have extraordinary mechanical, electrical, thermal, optical and chemical properties. These excellent characteristics render them ideal for a wide range of applications, such as catalyst support, ${ }^{79}$ energy conversion catalysts, ${ }^{80-83}$ biosensors ${ }^{84}$ etc. According to the layer of the wall, CNTs can be classified into single-walled carbon nanotubes (SWCNTs) and multi-walled carbon nanotubes (MWCNTs).

For modified CNTs to catalyze the ORR, Dai and co-workers did a series of pioneering and significant work in this field. ${ }^{16,85-90}$ For example, they synthesized a nitrogen-doped vertically aligned carbon nanotubes (VA-NCNTs) as an ORR catalyst (Fig. 2 (a) and (b)). The VA-NCNTs show comparable activity to that of the commercial Pt/C for the ORR in alkaline solutions (Fig. 2 (c)). The quantum mechanics calculations show that the improved ORR performance of the VA-NCNTs can be ascribed to the high electronegativity of $\mathrm{N}$ and the generation of positive charge on the surrounding $\mathrm{C}$ atoms. The modified carbon atoms enhanced the oxygen adsorption and readily attracted electrons from the anode to facilitate the ORR (Fig. 2 (d) and (e)). ${ }^{16}$ This study shows that the carbon atoms near the incorporated nitrogen are the possible active sites for the ORR. In addition, they employed a pure metalfree method fabricated another kind of NCNTs. ${ }^{86}$ As shown in Fig. 2 (f), a $\mathrm{SiO}_{2} / \mathrm{Si}$ wafer was firstly water-plasma treated to produce $\mathrm{SiO}_{2}$ nanoparticles as a catalyst on the substrate. This nanoparticles coated substrate was then transferred to a tubular furnace for the metal-free growth of CNTs by a chemical vapour deposition (CVD) method. The results show that compared to the unmodified CNTs 
synthesized by the same approach, the nitrogen modified sample NCNTs shows greatly improved ORR activity in an acidic solution. They claim that the ORR performance enhancement is exclusively due to the introduction of nitrogen into the tube structures. ${ }^{86}$ This result is consistent with their previous work on nitrogen modified CNTs for the ORR.
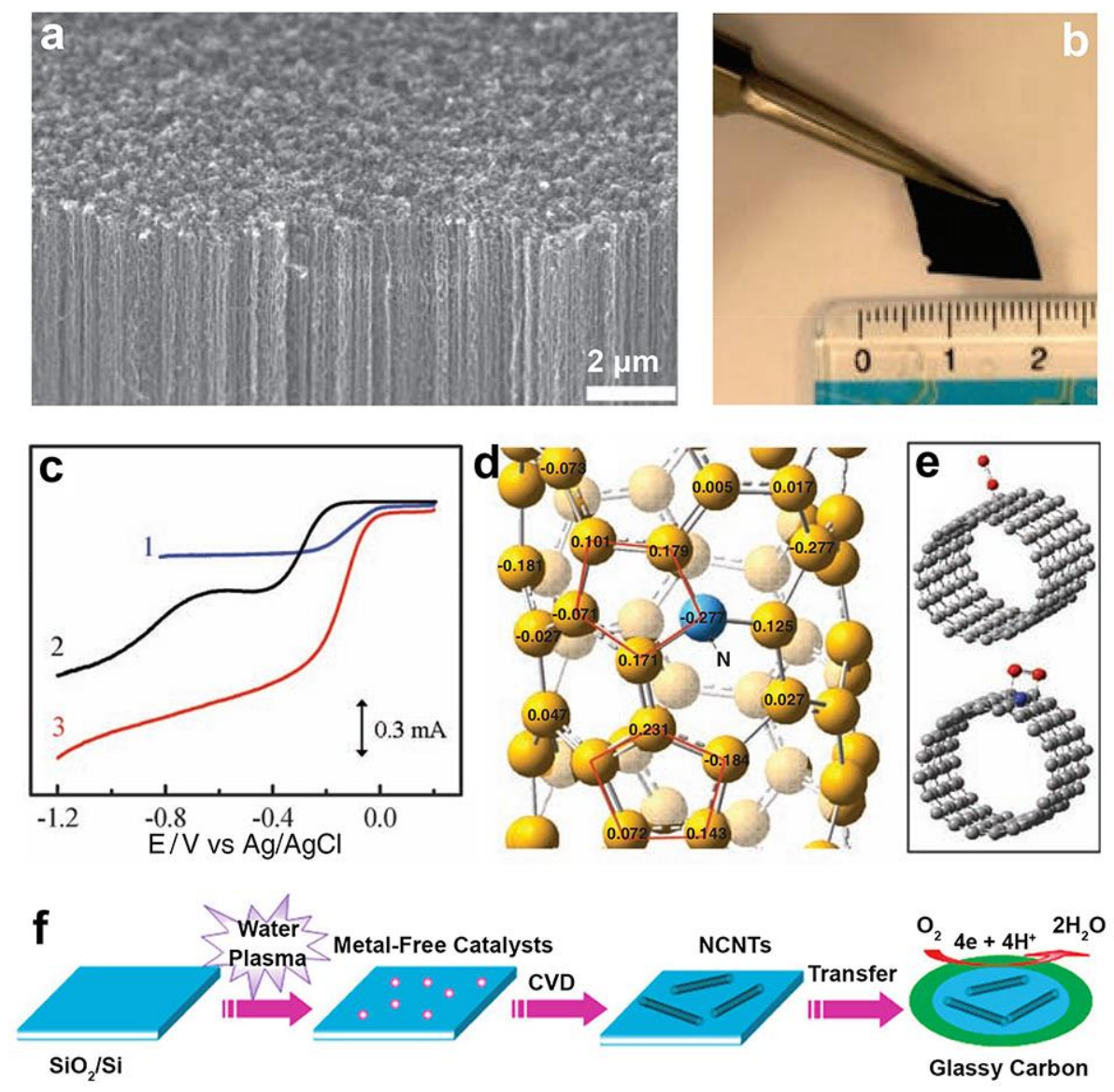

Fig. 2 (a) SEM image of the as-synthesized VA-NCNTs on a quartz substrate; (b) Digital photograph of the VANCNT array after having been transferred onto a PS-nonaligned CNT conductive nanocomposite film; (c) LSV curves of the prepared samples in an air-saturated $0.1 \mathrm{M} \mathrm{KOH}$ for Pt/C (curve 1), VA-CCNT (curve 2), and VA-NCNT (curve 3); (d) Calculated charge density distribution for the NCNTs; (e) Schematic representations of possible adsorption modes of an oxygen molecule at the CCNTs (top) and NCNTs (bottom). ${ }^{16}$ Reprinted with permission from ref. 16. Copyright 2009, AAAS. (f) Metal-free growth of nitrogen-doped CNTs for the ORR. ${ }^{86}$ Reprinted with permission from ref. 86 . Copyright 2010, American Chemical Society.

In addition to the work carried out by Dai and co-workers, other researchers also revealed that heteroatom modification plays vital roles in improving the ORR activity of the CNTs, especially with nitrogen. ${ }^{91-97}$ For example, via the density functional theory (DFT) simulations, Jiang et al. studied the reaction mechanisms of the ORR on graphite-like $\mathrm{N}$ groups $\left(\mathrm{N}_{\mathrm{G}}\right)$ and pyridine-like $\mathrm{N}$ groups $\left(\mathrm{N}_{\mathrm{P}}\right)$ 
nitrogen-doped carbon nanotubes (NCNTs). ${ }^{91}$ The calculations show that there is no big difference regarding the two-electron and four-electron pathways for the ORR at $\mathrm{N}_{\mathrm{G}}$ and $\mathrm{N}_{\mathrm{P}}$ defect sites in the lower potential range. However, at higher potentials, $\mathrm{N}_{\mathrm{P}}$ defect sites are more advantageous than $\mathrm{N}_{\mathrm{G}}$ over the four-electron reduction. It is also suggested that the reason why the pure CNTs show very low activities toward the ORR is that $\mathrm{OOH}$ cannot be adsorbed on the pure CNTs. ${ }^{91}$ In addition, Kauppinen and co-workers also applied the DFT model probed the origin of the ORR in the NCNTs. ${ }^{97}$ Based on their simulations, a kind of defect $\mathrm{N}_{4} \mathrm{~V}_{2} \mathrm{H}_{2}$ (N: N atoms, V: carbon vacancies, $\mathrm{H}$ : $\mathrm{H}$ atoms) is regarded to be the ORR active sites in the NCNTs. Besides, they found that the formation of defects and the binding energy of oxygen to the defects are related to the radius of the nanotubes. Only when the nanotube radius is large enough, then it will be effective for the ORR. ${ }^{97}$ This investigation further proves that the actual ORR active sites in the nitrogen modified CNTs are the defective carbons.
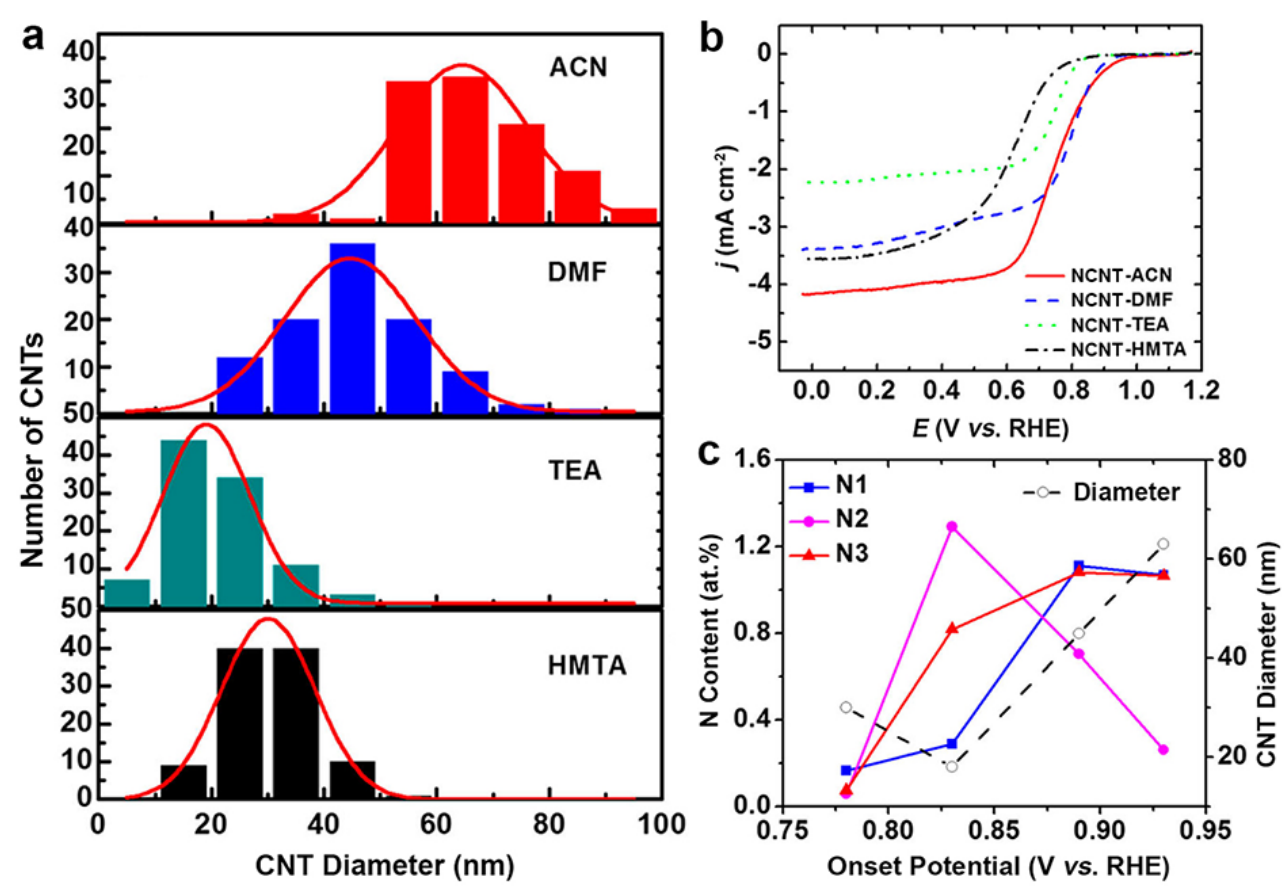

Fig. 3 (a) The influence of precursors on the diameter distribution of the prepared NCNTs; (b) Steadystate polarization curves comparison of the NCNTs that synthesized by different precursors; (c) The dependence of onset potential on $\mathrm{N}$ distribution and tube diameter (N1, N2, and N3 stand for pyridinic, pyrrolic, and graphitic nitrogen, respectively). ${ }^{98}$ Reprinted with permission from ref. 98 . Copyright 2015 , American Chemical Society.

Recently, this presumption was experimentally examined by Ajayan et al..$^{98}$ Through a liquid CVD method by elaborately selecting different nitrogen containing precursors, they successfully synthesized a series of NCNTs with variable diameters, as shown in Fig. 3 (a). The electrochemical test results in Fig. 3 (b) indicate that the prepared NCNTs with larger diameters are more favorable for the ORR. For 
example, NCNT-ACN with the largest diameter shows the most positive onset potential as well as highest current density compared to its smaller diameter counterparts (Fig. 3 (c)). They conclude that the larger diameter nanotubes could lower the oxygen adsorption energy, enhance the conductivity, and increase the nitrogen content in the NCNTs. All these modifications finally contributed to the high ORR activity. Besides, it reveals from Fig. 3 (c) that both pyridinic-N and graphitic-N are beneficial for the ORR. ${ }^{98}$ This result is in agreement with previous investigations that NCNTs with higher content of pyridinic-N show higher catalytic activity and selectivity toward the ORR. ${ }^{92,}{ }^{99}$ Moreover, Jousselme et al. also found that NCNTs with a relatively higher content of graphitic-N exhibit better ORR activities. ${ }^{100}$ In order to probe the active sites of the NCNTs and optimize the ORR performance, Wågberg et al. used a NCNT that contains six different types of nitrogen functionalities as the starting material, and fabricated a series of modified NCNTs. ${ }^{101}$ It is shown that the quaternary nitrogen (graphitic-N) located at the edge of the graphene planes is more active for the ORR, since the edges are enriched with carbon defects. These defect sites will facilitate the incorporation of nitrogen and accordingly the activation of the carbon atoms. In addition, the heat treatment process will unavoidably remove the doped nitrogen from the carbon structures. This process is highly possible to cause the rearrangement of the carbon atoms and subsequently form certain types of defects. Apparently, the newly created defects are quite possible beneficial for the ORR.

Besides nitrogen atoms, another heteroatom boron (B) was also utilized to modify the carbon materials for catalyzing the ORR. It is shown that B can accept electrons from carbon due to its three valence electrons configuration, which could shift the Fermi level to the conducting band. ${ }^{77}$ To dope B into the carbon structures, the frequently used methods are treating carbon precursors with B sources (such as boron powder ${ }^{102}$ and boric acid ${ }^{103}$ ) at high temperatures, or by the CVD approach ${ }^{76}$ For example, Hu et al. successfully introduced B into the CNTs through the CVD method. They found that the Bdoped CNTs show high ORR performance from the aspect of activity and stability. ${ }^{76}$ Inspired by this finding that B-doping could also effectively catalyze the ORR, Dai et al. employed a facile pyrolysis method prepared boron and nitrogen co-doped vertically aligned (VA-BCN) nanotubes, ${ }^{87}$ aiming to further improve its performance. As expected, the VA-BCN nanotubes exhibit higher ORR activity than that of the single B or N doped CNTs in an alkaline medium. Possibly due to the incorporation of both $\mathrm{B}$ and $\mathrm{N}$ into the CNTs produced a synergetic effect that is beneficial for the ORR ${ }^{87}$ However, the subsequent study carried out by Hu et al. revealed that not all B and N co-doped CNTs show improved ORR activity. The investigation shows that the ORR is also related to the dopants' configuration. ${ }^{104}$ Through well designed experiments, they successfully synthesized two kinds of B and N co-doped CNTs, one is dominated by bonded B and N, the other is feathered by separated B and N (illustrated in Fig. 4). Both the experimental and theoretical results show that it is very difficult to break the inert property of the CNTs when the doped B and N are bonded together. The possible reason is that the majority of the lone-pair electrons from the $\mathrm{N}$ dopant are neutralized by the vacant orbital from the $\mathrm{B}$ dopant. Therefore, 
the activation of carbon $\pi$ electrons is hardly to happen due to the lack of electrons or vacant orbitals. This is why it did not show improved ORR activity compared to the undoped CNTs. Besides, this investigation is in good agreement with previous DFT simulation results that $\mathrm{B}$ and $\mathrm{N}$ will be transparent to conduction electron if they are in a bonded state within the doped CNTs. ${ }^{105}$ On the contrary, the separated arrangement could interrupt the electroneutrality of the $s p^{2}$ carbon in a large extent. This alteration could create adequate active sites for oxygen absorption and reduction, thus it shows obviously improved ORR performance. ${ }^{104,105}$ Apparently, all these studies prove that if the heteroatoms could effectively alter the electronic structures of the adjacent carbon atoms, the modified carbon materials will effectively catalyze the ORR.
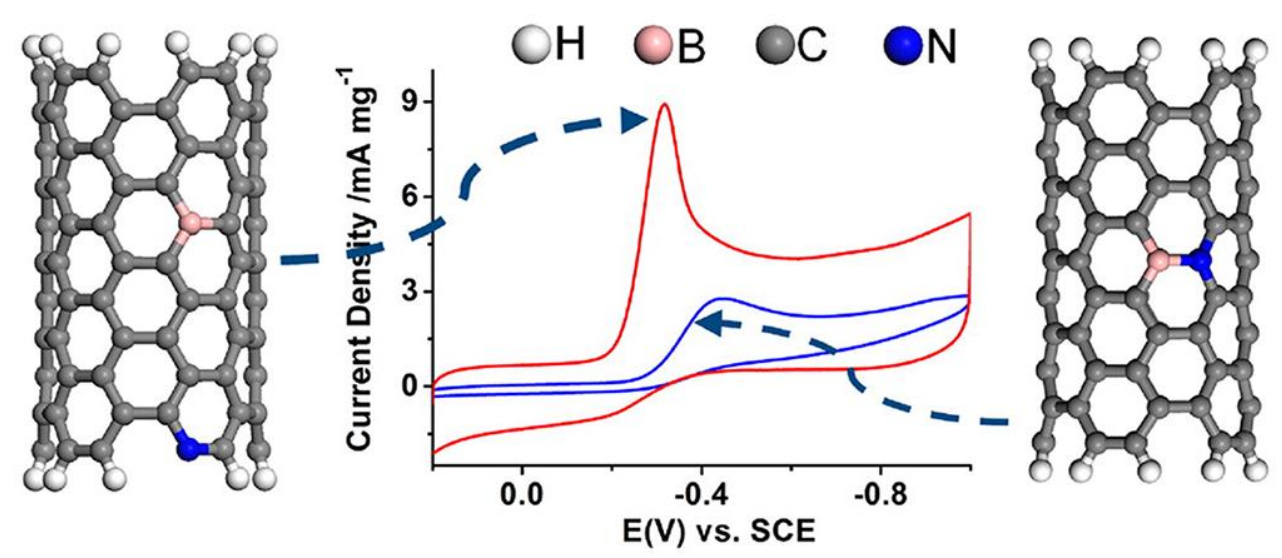

Fig. 4 ORR performance comparison of the B and N co-doped CNTs that dominated by bonded or separated B and N. ${ }^{104}$ Reprinted with permission from ref. 104. Copyright 2013, American Chemical Society.

Essentially, CNTs and graphene are the same material, only present in different configurations. In principle, it is possible to unzip the outer layer of the MWCNTs and develop a "new" material that couples with CNTs and graphene. This new composite material could take advantage of the unique physical and chemical properties of both CNTs and graphene, and may ideal for efficient ORR catalysis. It is interesting that Tour and co-workers successfully unzipped the CNTs by a simple solution-based oxidation method. Particularly, the opening extent of the CNTs can be controlled by tuning the amount of potassium permanganate $\left(\mathrm{KMnO}_{4}\right)$. For example, from partly unzipped CNTs to fully opened single layer graphene nanoribbons. ${ }^{106}$ Subsequently, they optimized the reaction conditions and fabricated high quality graphene oxide nanoribbons from the MWCNTs with less defects and holes on the basal plane. ${ }^{107}$ To extend the application of such new materials, Dai et al. used few-walled CNTs as the starting material, and then exfoliated their outer layer via an oxidation method. Afterwards, nitrogen was introduced into the complex under an ammonia atmosphere at a high temperature. ${ }^{108}$ It can be seen from Fig. 5 (a) and (b) that the outer walls of the CNTs are partially unzipped, and the formed small graphene sheets are 
connected to the intact inner walls of the CNTs. It is suggested that the synthesized complex with plenty of edge and defect spots is favorable to form catalytic sites for the ORR when treated with ammonia. As shown in Fig. 5 (c) and (d), the resulting sample carbon nanotube-graphene complex (NT-G) not only exhibits excellent ORR activity in alkaline media, but also shows remarkable ORR performance in acidic solutions. According to the atomic level investigations regarding the ORR active sites by a series of advanced characterizations, such as annular dark-field imaging and electron energy loss (EEL) spectrum imaging in an aberration-corrected scanning transmission electron microscopy (STEM), Xray photoelectron spectroscopy (XPS), and inductively coupled plasma mass spectroscopy (ICP-MS), it is found that both the doped nitrogen and the Fe impurities from the growth of CNTs are crucial to the ORR. Meanwhile, the novel structure of the catalyst is also indispensable. The unzipped and damaged outer walls of the CNTs could provide abundant defective sites for oxygen absorption and reaction, and the intact inner tubes are responsible for the rapid charge transfer during electrocatalysis process. ${ }^{108}$
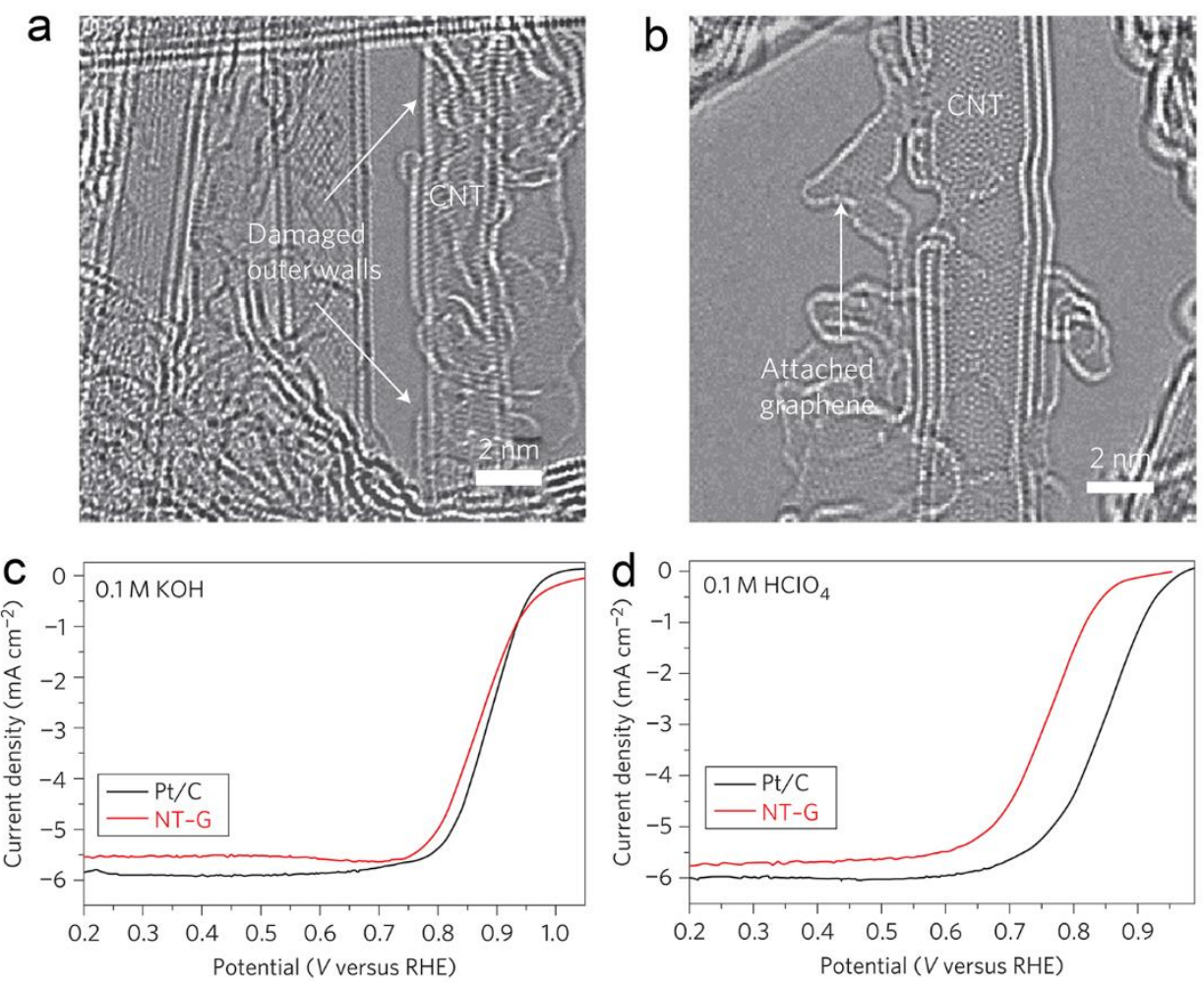

Fig. 5 (a) and (b) Aberration-corrected TEM images of the NT-G material, showing damaged outer walls and exfoliated graphene pieces attached to the double- or triple-walled CNTs; (c) and (d) RDE polarization curves of the Pt/C and NT-G in $\mathrm{O}_{2}$-saturated $0.1 \mathrm{M} \mathrm{KOH}$ and $0.1 \mathrm{M} \mathrm{HClO}_{4}$ solutions, respectively. ${ }^{108}$ Reprinted with permission from ref. 108. Copyright 2012, Springer Nature.

\subsection{Modified Graphene as Metal-Free Catalysts for the ORR}


As a new rising star, graphene has been received tremendous research attention due to its unique structure as well as its chemical, electronic, and mechanical properties. All these advantageous features render it as a promising candidate for a wide variety of applications. For example, it can used in the areas of electronics, biological engineering, photovoltaics, energy generation and storage. ${ }^{109-111}$ A case in point is as ORR catalysts. ${ }^{112-117}$ Similarly, tailoring the electronic structures of the graphene by heteroatoms modification should also be a feasible approach to fabricate highly active ORR catalysts for fuel cell applications.

Henrard et al. employed scanning tunnelling microscopy (STM) simulations studied the electronic properties of N and B modified graphene and CNTs. ${ }^{118}$ The predicted STM patterns show common fingerprints for graphene, metallic or semiconducting for CNTs. It is shown that the STM images are highly relevant to the local density of states of the carbon atoms near the doping defects. ${ }^{118}$ Recently, Ducastelle et al. investigated the structural and electronic properties N-modified graphene by the STM along with the DFT and the tight-binding calculations. They found that different arrangements of nitrogen impurities can be clearly detected, such as the single graphitic $\mathrm{N}$ atoms are presented as typical triangles. ${ }^{119}$ These investigations provide strong theoretical assistance for future experimental researches on chemically modified carbon nanomaterials via the STM. Besides, Hou et al. studied the influence of inherent point defects in graphene on the nitrogen doping by the DFT simulations from the aspect of energy profile. ${ }^{120}$ It is revealed that $\mathrm{N}$ doping in perfect graphene is endothermic, but it becomes exothermic in defective graphene. It also shows that the carbon atoms near the defect sites are more favourable to attract $\mathrm{N}$ atoms and energetically beneficial for the doping process. ${ }^{120} \mathrm{Xia}$ et al. applied the DFT method probed the ORR mechanism on N-modified graphene as well. It is illustrated that the introduction of nitrogen atoms could bring no-pair electrons to the graphene and modify its atomic charge distribution. This will subsequently account for the high ORR performance. ${ }^{121}$ It can be seen that after the modification of nitrogen, the resulting defective graphene is more active for the ORR.

The development of modern microscope technologies render it possible to capture some direct evidences regarding the electronic structures of the graphene at atomic levels, particularly after the modification of heteroatoms. For example, Bao et al. applied a one-step direct chemical method successfully synthesized N-modified graphene under solvothermal conditions. Then they observed the electronic perturbation in the graphene structures via the STM for the first time. The perturbation is caused by the introduction of nitrogen atoms. ${ }^{122}$ From Fig. 6 (a), it can be seen that the sample is a bilayer graphene, which is further confirmed by the high resolution STM images in Fig. 6 (b) and (c). In addition, this is also consistent with the previous reported results that the lattice constant of the small triangular grid motif is $2.4 \pm 0.1 \AA .{ }^{122-124}$ According to the analysis, the bright areas in the STM images were possibly induced by the incorporation of $\mathrm{N}$ atoms. The presence of defects and the electronic structure perturbation in the graphene network can also be observed from these STM images. The corresponding DFT simulations further supported this assumption from another angle, as shown in Fig. 
6 (c) and (d). ${ }^{122}$ The ORR test results show that the N-modified graphene exhibits obviously improved activity compared to the pristine graphene. This study demonstrates the crucial role of nitrogen in interrupting the graphene to create defective active sites for the ORR.
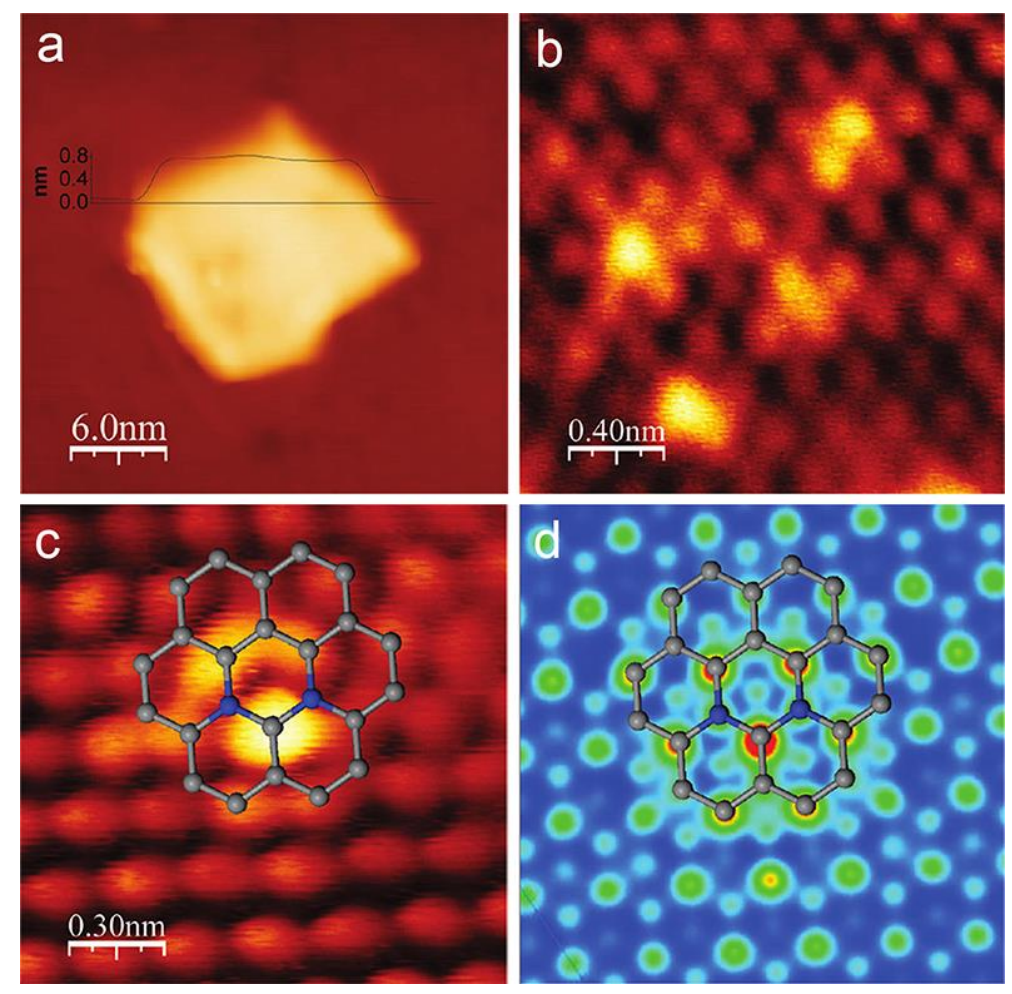

Fig. 6 (a) Isolated bilayer $\mathrm{N}$-doped graphene, the black curve on top showing the height measurement across this bilayer; (b) and (c) High resolution images with defects arranged in different configurations; (d) Simulated STM image for (c). The inserted schematic structures represent N-modified graphene. ${ }^{122}$ Reprinted with permission from ref. 122. Copyright 2011, American Chemical Society.

For preparing N-enriched graphene, many methods can be used. For example, the CVD method, ${ }^{58,}$ 125, 126 nitrogen plasma irradiation, ${ }^{127}$ post ammonia treatment, ${ }^{59,128,129}$ and through the pyrolysis of graphene oxide with nitrogen precursors. ${ }^{130-132}$ Ruoff et al. employed two different methods (ammonia treatment and $\mathrm{N}$-containing polymers pyrolysis) doped nitrogen into the graphene and investigated their influence on the ORR. It is shown that the bonding state of nitrogen has obvious influence on the ORR. Specifically, when treated the graphene under an ammonia atmosphere, it preferentially formed graphitic $\mathrm{N}$ and pyridinic $\mathrm{N}$. However, when utilizing polyaniline and polypyrrole as the nitrogen sources, it is more likely to produce pyridinic and pyrrolic N. It is revealed that the modified graphene with a higher content graphitic $\mathrm{N}$ shows higher limiting current density, while the one with higher pyridinic $\mathrm{N}$ exhibits more positive ORR onset potential. ${ }^{75}$ Obviously, different configurations of nitrogen in the carbon matrix will have different impact on the adjacent carbon atoms, thus it shows distinct ORR behaviours. 
In addition to the heteroatom $\mathrm{N}$, other foreign elements were also used to modify graphene to improve its ORR performance. As shown in Fig. 7, $\mathrm{N}$ or S-doped graphene was successfully fabricated by thermal reactions between graphene oxide and the guest gas $\mathrm{NH}_{3}$ or $\mathrm{H}_{2} \mathrm{~S}$ at high temperatures on the basis of graphene oxide-mesoporous silica sheets. ${ }^{133}$ Through this unique approach, the porous silica layer is able to facilitate the mass transportation during the thermal reaction process. Besides, it could also help to retain the layered graphene structures. It is shown that the resulting N-modified graphene and S-modified graphene exhibit good electrocatalytic activity and long durability as metal-free ORR catalysts. The ORR performance of those modified graphene is comparable to that of the commercial $\mathrm{Pt} / \mathrm{C} .{ }^{133}$ Apart from the traditional methods to prepare graphene from graphite, Liu et al. developed a novel one-step magnesiothermic reduction approach synthesized a crumpled S-doped graphene with a few layers. ${ }^{134}$ The prepared S-modified graphene shows more positive onset potential and higher limiting current density than those of the pure graphene. They pointed out that the high ORR activity of the prepared catalyst is due to its graphitized and hierarchical porous structures. These unique structures could provide abundant sites to absorb and reduce oxygen after the modification of sulfur. ${ }^{134}$
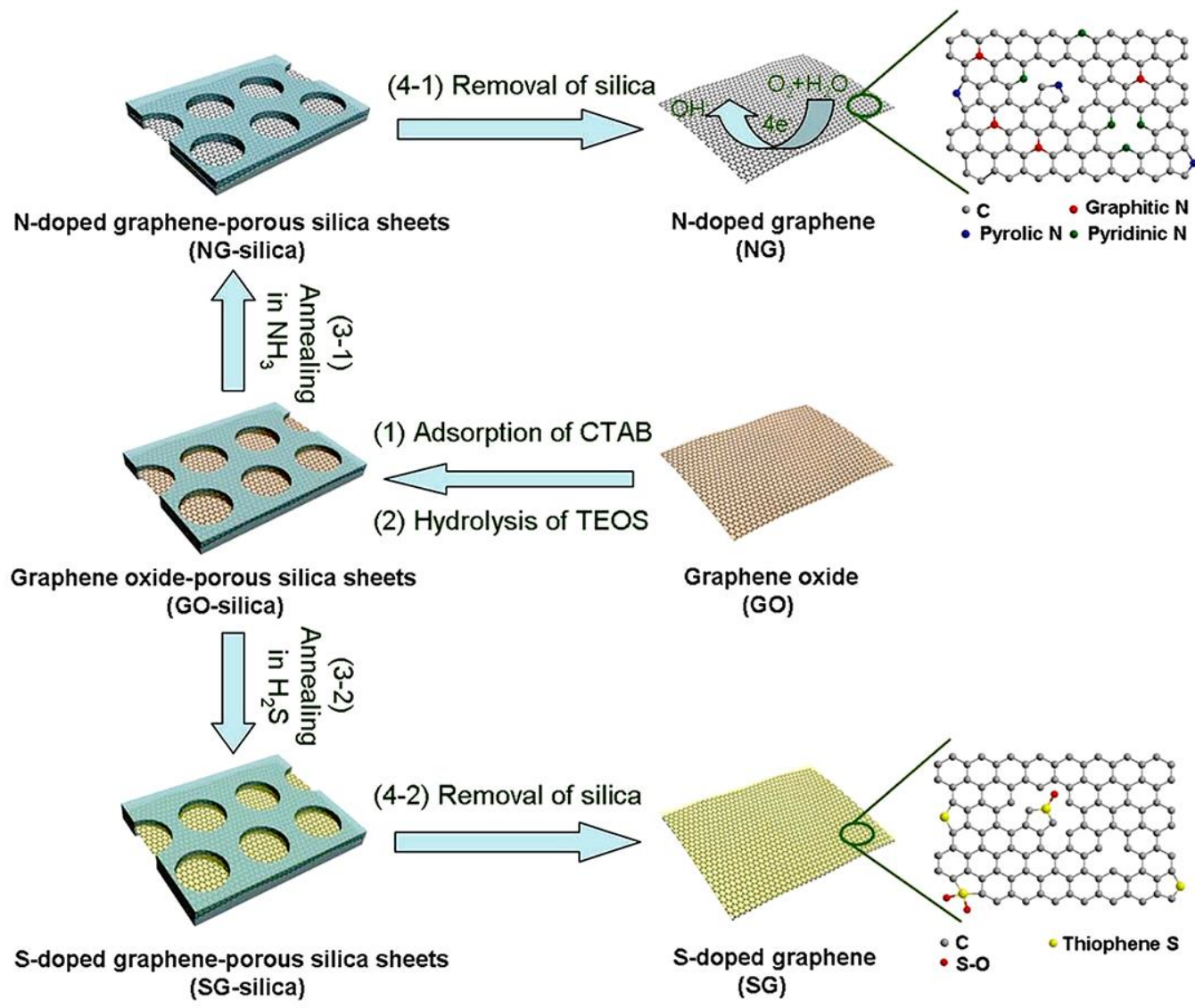

Graphene oxide

(GO)

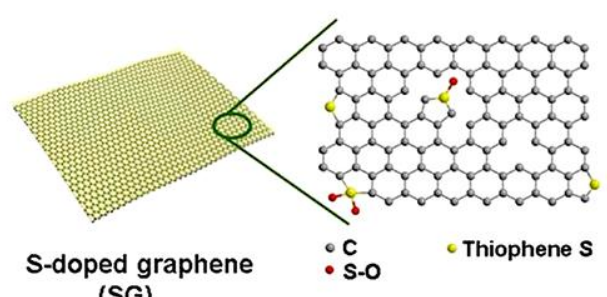

(SG)

Fig. 7 Schematic illustration showing the synthesis of N and S-doped graphene. ${ }^{133}$ Reprinted with permission from ref. 133. Copyright 2012, John Wiley and Sons. 
According to the work conducted by Huang and co-workers on S-doped graphene, the high ORR activity of heteroatoms modified carbon nanomaterials may not derive from the electronegativity differences between the carbon atoms and the foreign elements. This is because the electronegativities of carbon and sulfur are very close (2.55 vs 2.58), but the S-doped graphene still exhibits excellent ORR performance. ${ }^{135}$ Therefore, alternative mechanisms, such as the defects promoted catalysis mechanism can be used to explain the ORR performance improvement for the $\mathrm{S}$ modified graphene. For example, Baek et al. applied theoretical calculations investigated the origin of S-functionalized graphene. It is proposed that the introduced sulfur atoms and sulfur oxides could alter the electronic spin density and the charge density of the surrounding carbon atoms significantly. This modification plays important roles in promoting the ORR. ${ }^{136}$ Furthermore, Xia and co-workers also probed the ORR active sites and reaction mechanisms of S-doped graphene by the DFT calculations. ${ }^{137}$ It is revealed that the ORR active sites are those carbon atoms located at the graphene edges or near the doped $\mathrm{SO}_{2}$ sites. Those centers exhibit high positive charge density or spin density after the incorporation of $S$, and thus could favor the high efficient four-electron ORR pathway. ${ }^{137}$ Apparent, the catalysis mechanism based on defect structures is a general mechanism to explain the heteroatoms modified carbons for the ORR.

Besides the modification of graphene by $\mathrm{N}$ or $\mathrm{S}$, the halogenated carbon materials, particularly graphene also show enhanced ORR activity. ${ }^{138-143}$ For example, Dai and co-workers employed a ballmilling method successfully prepared various edge-selectively halogenated (chlorine $(\mathrm{Cl})$, bromine $(\mathrm{Br})$ and iodine (I)) graphene nanoplatelets. ${ }^{138}$ It is shown that all the three halogenated graphene catalysts exhibit improved ORR performance compared to the graphite. Meanwhile, the iodine modified graphene nanoplatelets show the highest ORR activity among them. This is because the incorporation of iodine into the graphene edges render the modified graphene most effectively attracting the oxygen molecules and subsequently weakening the $\mathrm{O}-\mathrm{O}$ bond. ${ }^{138}$ In addition, they also fabricated a N, P and F tri-doped graphene via the pyrolysis of polyaniline-coated graphene oxide in the presence of ammonium hexafluorophosphate. The synthesized electrocatalyst shows excellent ORR performance in alkaline solutions. This is due to the surface properties alterations and the introduction of defects into the graphene sheets during the incorporation of the heteroatoms. ${ }^{141}$ As can be seen, this investigation also supports the effective role of defects in promoting the ORR.

\subsection{Modified Mesoporous Carbons for the ORR}

The development of ordered porous carbon materials with controllable structures is another promising strategy to promote the ORR. Particularly after the modification of heteroatoms, such as N, $\mathrm{P}, \mathrm{B}, \mathrm{S}, \mathrm{F}$ and $\mathrm{O}$. The investigations indicate that compared to other configurations, the ordered porous structures could provide more active sites for the oxygen absorption and reaction. ${ }^{144}$ The contribution of mesopores $(2-50 \mathrm{~nm})$ for the mass transportation of ORR-relevant species may also facilitate the 
ORR. ${ }^{145}$ This is owing to the multiple advantages of mesoporous materials, including high specific surface area, adjustable pore size, and large pore volume with narrow pore size distributions. ${ }^{146-150}$ Therefore, many mesoporous carbon-based materials have been synthesized as ORR catalysts in recent years. Typical examples include $\mathrm{N}$-modified mesoporous carbon spheres, ${ }^{151-153} \mathrm{~N}$-modified carbon nanosheets with size-defined mesopores ${ }^{154} \mathrm{~N}$-modified mesoporous graphene, ${ }^{155}$ ordered mesoporous carbon nitrides, ${ }^{156} \mathrm{~B}$-modified mesoporous carbon, ${ }^{157} \mathrm{P}$-modified mesoporous carbon, ${ }^{158}$ and N/S, ${ }^{159,}, 160$ $\mathrm{N} / \mathrm{O},{ }^{161} \mathrm{~N} / \mathrm{F}^{140}$ co-modified carbon materials.

Müllen et al. fabricated a series of N-modified carbon nanosheets (NDCN) with uniform and adjustable mesopores by using a template method, as schemed in Fig. 8 (a). ${ }^{154}$ Fig. 8 (b) shows a TEM image of the graphene-based silica nanosheets that sandwiched with close-packed colloidal silica with the particle size of $22 \mathrm{~nm}$ (G-silica-22). From which, we can see that the silica spheres are homogeneously attached to the surface of the graphene-based silica nanosheets (G-silica). The mesoporous NDCN with the desired pore size $(22 \mathrm{~nm})$ was successfully synthesized after removing the silica template (Fig. 8 (c)). By the same approach, they also prepared NDCN with other pore sizes, such as $7 \mathrm{~nm}$. The electrochemical performance test results show that the resulting NDCN with the pore size of $22 \mathrm{~nm}$ exhibits comparable ORR performance to that of the commercial $\mathrm{Pt} / \mathrm{C}$ in alkaline media. It can be attributed to the special planar mesoporous shell structures that could provide sufficient active sites on the NDCN surface and boost the electrolyte/reactant diffusion during the ORR process. ${ }^{154}$ Interestingly, $\mathrm{Yu}$ and co-workers applied a templateless method fabricated a N-modified mesoporous graphene. The graphene was synthesized by treating the mixture of the pre-synthesized graphene oxide and polydopamine at high temperatures directly. ${ }^{155}$ The polydopamine not only acting as the nitrogen source, but also efficiently preventing the re-aggregation of the graphene nanosheets during the pyrolysis process. The investigation shows that the prepared sample exhibits obviously improved ORR activity compared to the reference samples (without well-defined structures). This can be ascribed to its high specific surface area, mesoporous architecture as well as the modification of the nitrogen. ${ }^{155}$ 

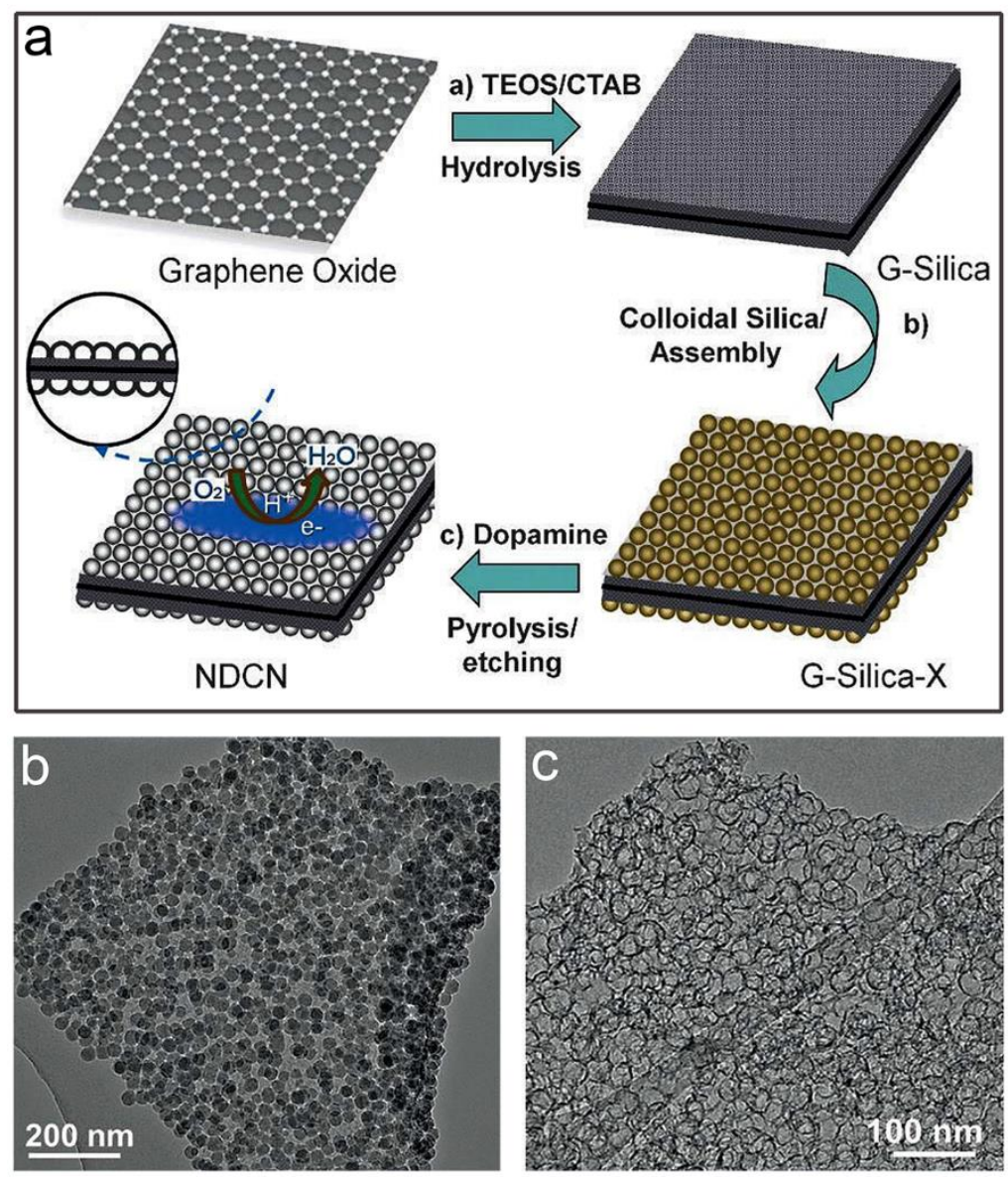

Fig. 8 (a) Schematic illustration the preparation of nitrogen-modified carbon nanosheets (NDCN) with the desired pore size; (b) Typical TEM image of G-silica-22, revealing the uniform adhesion of silica22 on the surface of G-silica; (c) Morphology and microstructure of the resulting NDCN-22. ${ }^{154}$ Reprinted with permission from ref. 154. Copyright 2014, John Wiley and Sons.

In addition, Yu et al. successfully synthesized P-modified ordered mesoporous carbons (POMCs) with different lengths. Thereafter, they investigated the influence of the length of the porous carbons on the ORR in alkaline solutions. ${ }^{158}$ It is found that the prepared POMC with shorter rod length exhibits higher ORR activities. This is possibly due to its higher surface area and the lower resistance of the shorter channels. Besides, it is shown that the introduction of $\mathrm{P}$ into the ordered structure is also crucial to the ORR. As suggested, the incorporation of P may induce defects in the carbon network and disturb the electronic structures owing to its high electron-donating property. This alteration could facilitate the oxygen adsorption and subsequently promote the ORR. ${ }^{158,162,163}$ Recently, Lee et al. proposed that the POMC with larger pore size (POMC-L) is more beneficial to improve the cell performance. They used the pre-prepared POMC-L as the starting material, and then further treated it under different conditions. This treatment is to adjust the nitrogen doping site positions to reach a better electrocatalytic performance, as schemed in Fig. 9 (a). ${ }^{144}$ Fig. 9 (b) and (c) show the TEM and SEM images of the 
prepared N-doped POMC-L (NPOMC-L2). It is obvious that the resulting sample exhibits very uniform and ordered mesoporous structures. The single-cell test results prove that the N, P co-modified sample NPOMC-L2 with larger pore size and properly controlled nitrogen doping sites shows significantly enhanced onset potential compared to the undoped sample with smaller pore size. Remarkably, the power density of the NPOMC-L2 can reach as high as $70 \%$ of the commercial Pt/C, as shown in Fig. 9 (d). ${ }^{144}$ This study suggests that the pore size of the electrocatalysts is directly related to the cell performance. Therefore, it is a viable option to tune the catalytic activity of the electrocatalysts by adjusting their pore size. In addition, the active sites can be easily accessed by the reactants if they are located in the mesoporous region. This will increase the mass transport efficiency and realize a high utilization of the catalyst layer.

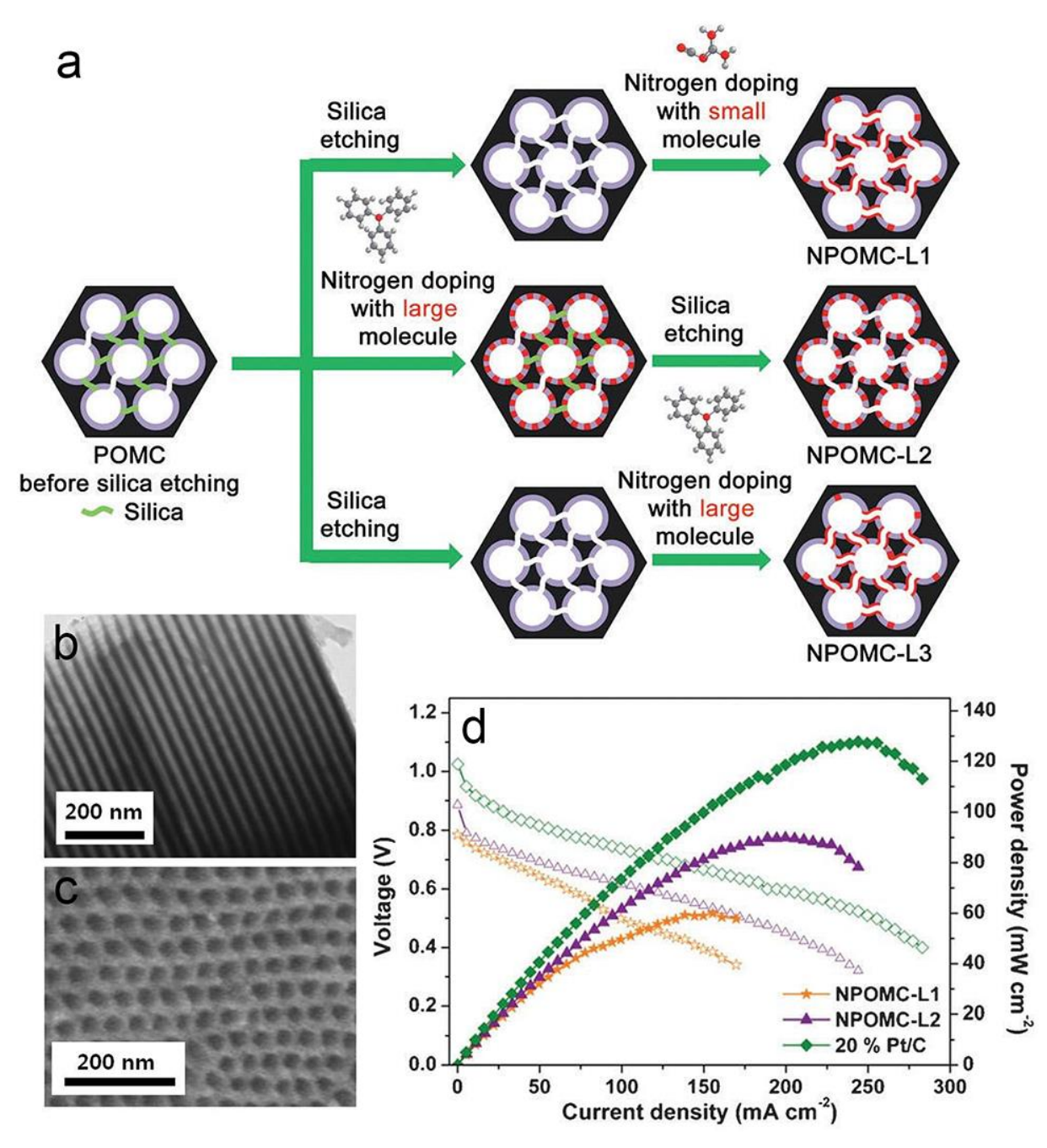

Fig. 9 (a) Schematic representation of the synthetic conditions for additional nitrogen doping into the POMC-L; (b) and (c) TEM and SEM images of the prepared NPOMC-L2; (d) Polarization curves of MEAs at $60{ }^{\circ} \mathrm{C}$ (open and closed symbols correspond to cell voltage and power density, respectively). ${ }^{144}$ Reprinted with permission from ref. 144. Copyright 2015, John Wiley and Sons. 


\section{Intrinsic Carbon Defects Promoted ORR}

From the above discussions, it is obvious that the nature of heteroatoms doping to improve the ORR activity of carbon materials is most likely to activate the adjacent carbon atoms to make them active for the ORR. It means that the real ORR active sites may be the carbon atoms themselves. Theoretically, this is a big breakthrough in revealing the reaction mechanisms of electrocatalysis, such as on the ORR active sites of metal-free electrocatalysts. Following this newly established catalysis mechanism based on defect structures, many issues regarding the heteroatoms doping, particularly N-doping, are now can be solved. For example, the relationship between the nitrogen concentration and the ORR performance of the N-doped carbon materials used to be an ongoing debate, ${ }^{19,}$ 59, 72, 96, 131, 164-167 but now it can be explained by using the proposed defects driven catalysis mechanism. This is because the incorporated nitrogen is not the active sites for the ORR, but providing an approach to alter the electronic structures of the surrounding carbon atoms. Therefore, the concentration of nitrogen is not directly related to the ORR performance. Comparatively, how the introduced nitrogen affects the carbon electronic structures is more important in promoting the ORR. Similarly, the argument on whether the graphitic N or the pyridinic $\mathrm{N}$ is more active for the $\mathrm{ORR}^{59,92,99,100,168,169}$ can also be clarified by applying the defects promoted catalysis mechanism. From recent advancement on developing various defective carbons for the ORR, ${ }^{36,61-66}$ it can be found that any methods that could alter the electronic configurations of the carbon atoms are also capable of promoting the ORR. The synthesized defective carbons are possibly more effective than the heteroatoms modified carbons to catalyze the ORR. This section will summarize recently published work on intrinsic carbon defects promoted ORR.

\subsection{A Defects Driven Catalysis Mechanism for the ORR}

In recent years, a generally accepted defects driven catalysis mechanism has be established based on the extensive research on uncovering the veil of the active sites in metal-free ORR electrocatalysts. ${ }^{40,}$ ${ }^{53-58}$ Particularly, a series of pioneering work has been carried out by Yao and co-workers to probe the ORR active sites in metal-free ORR catalysts. ${ }^{26,36,61-63}$ Gradually, it is realized that carbon defects are the actual active sites in various modified carbon-based ORR catalysts. The first systematic work on defects promoted ORR was published in early 2015. It is shown that a porous carbon material with newly created defects is an efficient ORR catalyst. It could catalyze the ORR more efficiently than the nitrogen modified carbons. ${ }^{61}$ First of all, the theoretical simulations were applied to probe the reaction pathways of the defective carbon. To simplify the calculations, a stable and representative divacancy G585 (contains two pentagons and one octagonal) defect has been selected for the modelling. ${ }^{170-176}$ The structure of the G585 defect can be found in Fig. 10 (a). ${ }^{61}$ Herein, four catalysts have been calculated in terms of energy profile using the DFT models. It can be observed from Fig. 10 (b) that the incorporation of nitrogen into the graphene could dramatically lower the adsorption energy of oxygen. However, it is unfavourable for the following reactions compared to the ideal catalyst. For example, the reaction barrier of reducing the chemisorbed oxygen atoms is relatively high. In contrast, graphene with G585 defect 
not only could facilitate the adsorption of oxygen, but also could lower the energy barrier for the following reactions. It is comparable to that of the ideal ORR catalyst Fig. 10 (b). This means that pure carbon materials with certain types of defects can be used as highly effective ORR catalysts. Subsequently, this conclusion was examined experimentally by fabricating defective carbons from a Ncontaining porous aromatic framework (PAF-40). The evenly distributed nitrogen in the PAF-40 sample was removed by a high temperature treatment. Naturally, various defects, such as the G585 are quite possible to be formed during the nitrogen removal process. By treating the PAF-40 at elevating temperatures, the nitrogen contents were decreased gradually in the resulting samples, as proved by the XPS analysis (Fig. 10 (c)). The corresponding Raman spectra in Fig. 10 (d) show the generation of defects in the carbon samples. As expected, the samples with less nitrogen show more positive ORR onset potentials and near-four electron-transfer number (Fig. 10 (e) and (f)). ${ }^{61}$ It is suggested that the excellent ORR performance of the resulting samples can be attributed to the newly created topological defects by removing the nitrogen atoms from the carbon matrix. In addition, this experimental result is a strong support to the corresponding theoretical simulations.
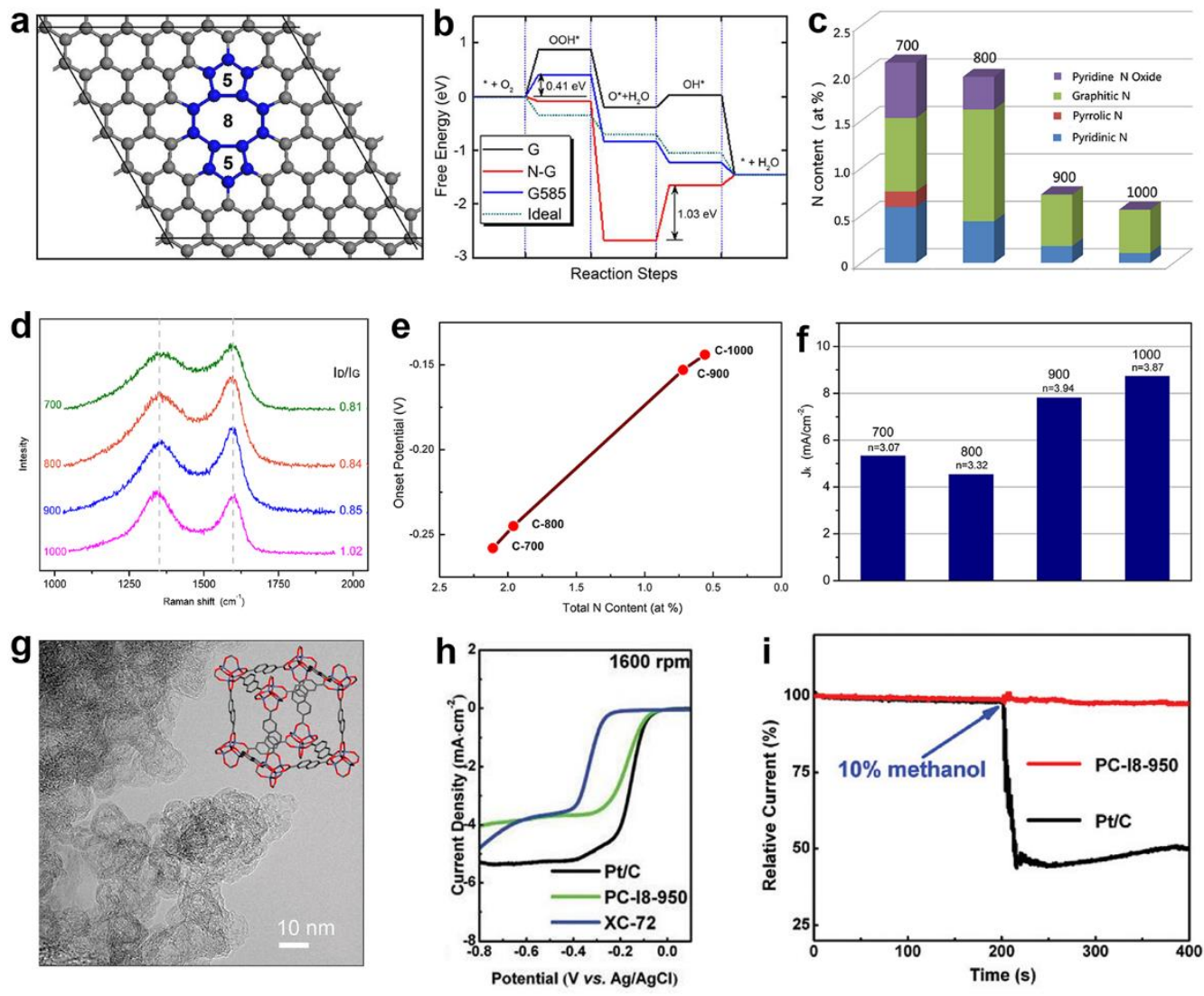

Fig. 10 (a) Pictorial representation of the G585 defect in graphene; (b) Calculated free energy diagram of perfect monolayer graphene (G), N-doped graphene (N-G), graphene with G585 defect (G585) and an ideal catalyst (Ideal) for the ORR at the equilibrium potentials; (c) Relative atomic percentage of different nitrogen bonding states in the prepared samples; (d) Raman spectra of the synthesized samples; (e) The relationship between the nitrogen contents and the onset potentials of the samples; (f) Kinetic 
current densities and the electron-transfer number of the prepared samples. ${ }^{61}$ Reprinted with permission from ref. 61. Copyright 2015, The Royal Society of Chemistry. (g) HRTEM image of the sample PCI8-950 (Inset: Structure of IRMOF-8. ${ }^{177}$ Reproduced from Ref. 177 with permission from The Royal Society of Chemistry.); (h) LSV curves at $1600 \mathrm{rpm}$ of PC-I8-950, Pt/C and XC-72 catalysts; (i) Methanol tolerance test of the PC-I8-950 sample and the commercial Pt/C. ${ }^{62}$ Reprinted with permission from ref. 62. Copyright 2016, The Royal Society of Chemistry.

For electrocatalysis, trace impurities, either metals or non-metals are possible the actual active sites for the catalytic reactions. For examples, the major contributor of many reported "metal-free" ORR electrocatalysts may be the trace metal impurities. ${ }^{178-180}$ It is very hard or even impossible to completely remove the catalyst Fe (normally used for the growth of CNT) from CNTs, since it is embedded or coordinated with the carbon atoms. The PAF-40 derived defective carbon C-1000- $\mathrm{O}_{2}$ still contains 0.21 at.\% nitrogen. Although it shows very clear activity-N relationship (the lower the $\mathrm{N}$, the higher the activity), it is reasonable to eliminate the possible effect of the trace amount of residual $\mathrm{N}$ on the ORR. In this regard, Yao and co-workers designed another experiment to fabricate $\mathrm{N}$-free defective carbons. Specifically, a nitrogen-free Zn-enriched metal organic framework (IRMOF-8, the structure can be seen from the inset of Fig. $10(\mathrm{~g})$ ) was carbonized at high temperatures. ${ }^{62}$ As Zn has a low boiling point of $\sim 907{ }^{\circ} \mathrm{C}$, it will be removed through evaporation when being heated at a temperature higher than its boiling point. For example, the containing $\mathrm{Zn}$ was completely removed at a temperature of $950{ }^{\circ} \mathrm{C}$, as verified by the XPS analysis (also no nitrogen is detected). The removal of $\mathrm{Zn}$ is similar to the removal of $\mathrm{N}$ from the carbon structures, thus it is highly possible to produce the effective defects to promote the ORR. As expected, the resulting defective porous carbon PC-I8-950 (Fig. 10 (g)) exhibits obviously improved ORR performance (Fig. 10 (h)). Remarkably, the onset potential is even comparable to that of the commercial Pt/C, and it is free from methanol poisoning (Fig. 10 (h and i)). This study fully suggests that it is not the impurities ( $\mathrm{N}$ or $\mathrm{Zn}$ ) but the newly created carbon defects via the removal of the $\mathrm{Zn}$ atoms from the IRMOF-8 structures facilitated the ORR. ${ }^{62}$ Apparently, this investigation is a strong support and complementary to the proposed defects driven catalysis mechanism. In addition, it also shows that apart from the nitrogen atom, the removal of other heteroatoms (including metal atoms) from the carbon structures could create the effective defects for the ORR as well. Therefore, it is a general method to produce defective carbons by removing heteroatoms from the carbon matrix.

More in-depth and systematical theoretical investigations have been carried out by Xia et al. on defects promoted ORR. They probed the electronic structure and ORR performance of graphene with various point and line defects via the DFT calculations. ${ }^{64}$ Their simulation results show that both the point and line defects in graphene could alter the local electronic configurations and charge distributions of nearby carbon atoms. These modifications are possible to make the defective graphene for efficient 
ORR catalysis. It is found that only particular types of point defects, such as the one has a pentagon ring located at the zigzag edge could catalyze the ORR (PZ, Fig. 11 (a) inset). In addition, according to their calculations, the odd number of octagon rings and fused pentagon rings line defects at the edge of the defective graphene (GLD-558-01, Fig. 11 (b) inset) are the proposed ORR active sites as well. It means that the combination of certain types of defects and the edge structures are contributing to the excellent ORR activity of the defective graphene. Fig. 11 (a) and (b) are the calculated free energies at different potentials for the graphene with PZ and GLD-558-01 defects, respectively. It can be observed that graphene with the GLD-558-01 defects shows higher ORR activity than that of with the PZ defects. This is consistent with the reported theoretical simulation results. ${ }^{3,181,182}$ Remarkably, in terms of energy barriers, the defective graphene shows similar ORR activity to the $\operatorname{Pt}(111)$ surface ${ }^{183}$ and nitrogen modified porous carbons. ${ }^{60}$
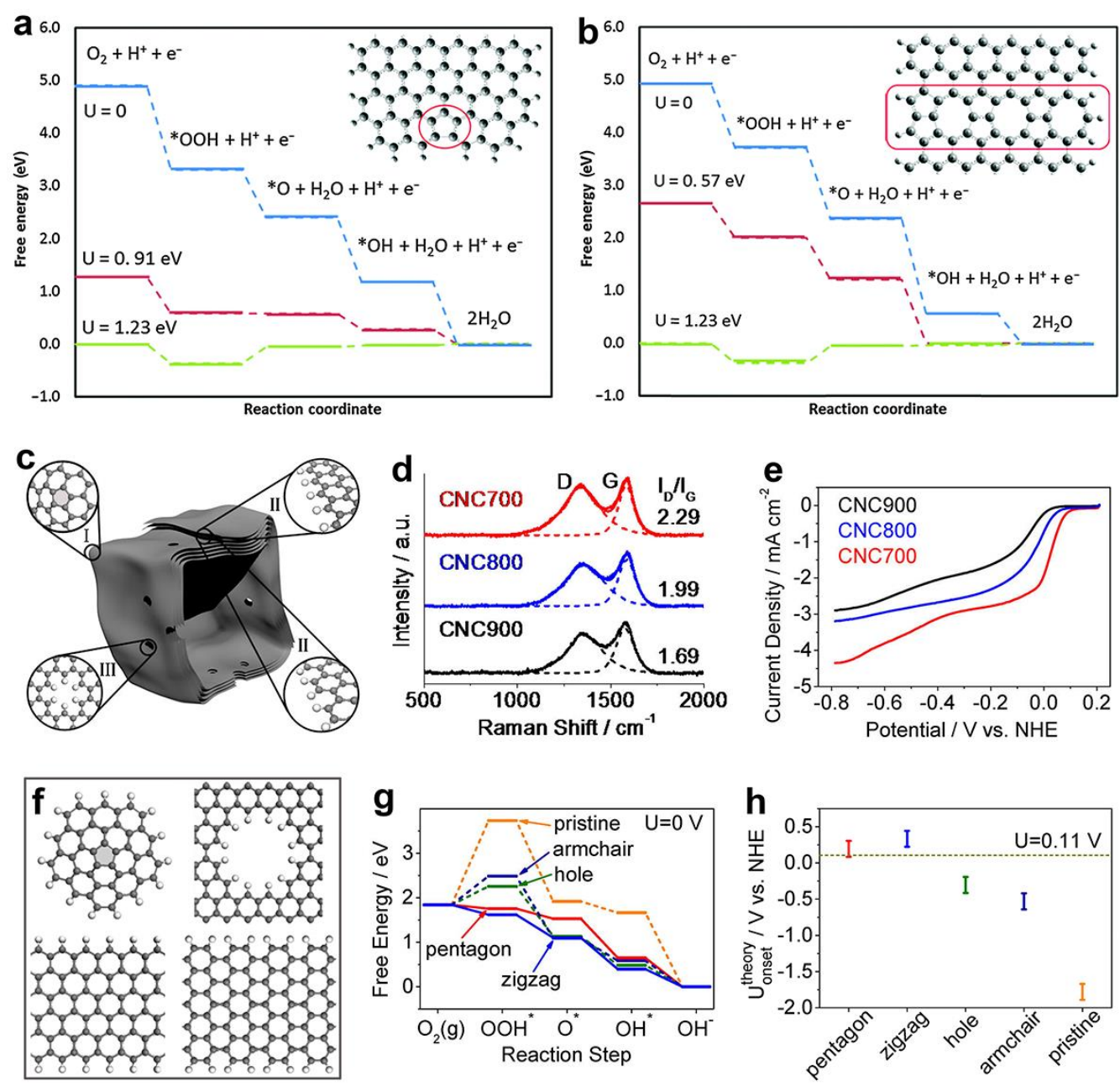

Fig. 11 (a) Defective graphene cluster with pentagon ring at the zigzag edge (PZ) and the corresponding calculated reaction free energy diagram for the four-electron transfer ORR process; (b) Defective graphene cluster with odd number of octagon rings and fused pentagon rings line defect (GLD-558-01) and the corresponding calculated reaction free energy diagram for the four-electron transfer ORR process. ${ }^{64}$ Reproduced from Ref. 64 with permission from the PCCP Owner Societies. (c) Schematic 
structural characters of the carbon nanocages. I, II, and III represent three typical defective locations (the corner, the broken fringe, and the hole, respectively); (d) Raman spectra of the prepared samples; (e) LSV curves of CNC700-, CNC800-, and CNC900-tested in $\mathrm{O}_{2}$-saturated $0.1 \mathrm{M} \mathrm{KOH}$ solution with the rotating speed of $2500 \mathrm{rpm}$; (f) Defect models used for theoretical calculations. Pentagon (highlighted), hole, zigzag edge and armchair edge; (g) Free energy diagrams derived from the DFT calculations for ORR activity of different defects; (h) Theoretically predicted and experimentally observed onset potentials of the defective carbon nanocage CNC700. ${ }^{65}$ Reprinted with permission from ref. 65. Copyright 2015, American Chemical Society.

$\mathrm{Hu}$ and co-workers subsequently studied the influence of the intrinsic carbon defects on the ORR, both experimentally and theoretically. ${ }^{65}$ They prepared a dopant-free carbon nanocage enriched with various intrinsic carbon defects, including the cage corner pentagon defects, the broken fringe edge defects, and the holes in the shell, as shown in Fig. 11 (c). The nanocages synthesized under different temperatures show different defective characters, as indicated by the corresponding Raman spectra in Fig. 11 (d). Among the fabricated defective nanocages, the CNC700 (treated at $700{ }^{\circ} \mathrm{C}$ ) shows the highest $I_{\mathrm{D}} / I_{\mathrm{G}}$ ratio. It means that the $\mathrm{CNC700}$ contains the largest amount of defects. The corresponding electrochemical test results show that the $\mathrm{CNC700}$ also exhibits the most positive onset and half-wave potentials among the three samples (Fig. 11 (e)). These results fully demonstrate the crucial role of the intrinsic carbon defects in promoting the ORR. Besides, the ORR activity of the CNC700 is comparable or even better than that of the reported nitrogen modified carbon materials. ${ }^{58,184-186}$ Afterwards, they established four models (pentagon, hole, zigzag edge and armchair edge) for the DFT calculations (Fig. 11 (f)). The calculated energy profiles in Fig. 11 (g) shows that the pentagon and zigzag edge defects are more active than other types of defects. This result is further supported by the theoretically predicted onset potential ranges for the modelled four defects, as shown in Fig. 11 (h) ${ }^{65,}{ }^{187}$ This study provides deep insight into the origin of the ORR in dopant free carbon materials. It is revealed that certain types of intrinsic carbon defects, such as the pentagon and zigzag edge defects are responsible for the efficient ORR catalysis. This finding could be beneficial for designing active metal-free electrocatalysts for practical applications.

Although tremendous investigations have been devoted to establishing a catalysis mechanism based on defect structures for the ORR, the direct observation of the proposed defects, such as the pentagon and G585 defects is still very difficult. This is because most of the prepared defective carbons are present in three-dimensional porous structures. The complexity of the defective samples makes them unsuitable for the TEM characterizations. In order to gain direct evidences regarding the formation of the proposed effective defects in the defective carbons, Yao and co-workers thereafter selected a single layer graphene as a model material for fabricating defective graphene (DG). As schemed in Fig. 12 (a), a facile nitrogen 
doping and removal approach was used to prepare the DG. ${ }^{36}$ It can be seen from the corresponding Raman spectra in Fig. 12 (b) that compared to the introduction of nitrogen into the pristine graphene, more defects are generated with the removal of the doped nitrogen. It means that the removal of the heteroatoms is more effective in producing defects in carbon materials than the introduction of the heteroatoms into carbons. The atomic force microscopy image of the DG shows that the DG is single layer (Fig. 12 (c)). As expected, various defects can be clearly observed from the DG with the assistance of the aberration-corrected high-resolution TEM (Fig. 12 (d)). For example, the afore-mentioned edge pentagons and the G585 defect. In addition, other types of defects, such as the 75585 and 5775 defects can also be found in the DG. The direct observation of the effective defects in the DG provides a solid evidence to support the proposed defects driven catalysis mechanism. Besides, it is also a strong support to the theoretical simulations. From the electrochemical test results in Fig. 12 (e) to (g), it can be found that the DG not only shows greatly enhanced ORR activity, but also an ideal catalyst for the oxygen evolution reaction (OER) and hydrogen evolution reaction (HER). These results prove the versatility of the defects driven catalysis mechanism for electrocatalysis. The corresponding DFT calculations show that the most active configuration is the edge 5-1 defect for the ORR in alkaline electrolytes (Fig. 12 (h) to $(\mathrm{k})$ ). It is consistent with the previous modellings and experimental results. ${ }^{61,64,65}$ Therefore, it is a feasible method to design efficient ORR catalysts by creating the unique defects for practical fuel cell applications. 

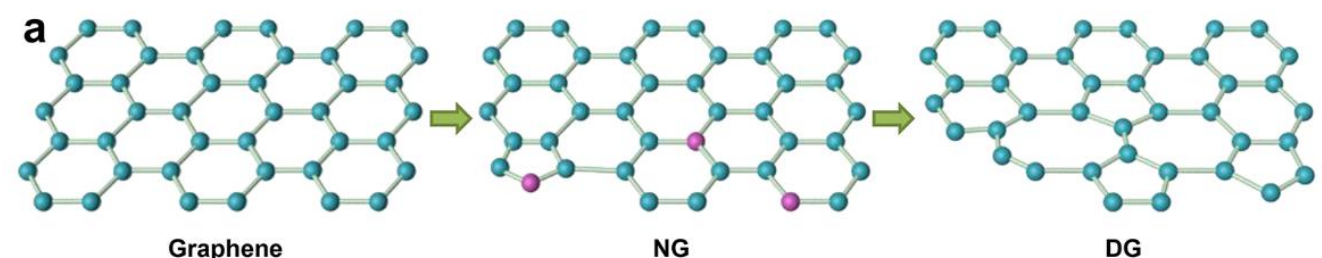

Graphene
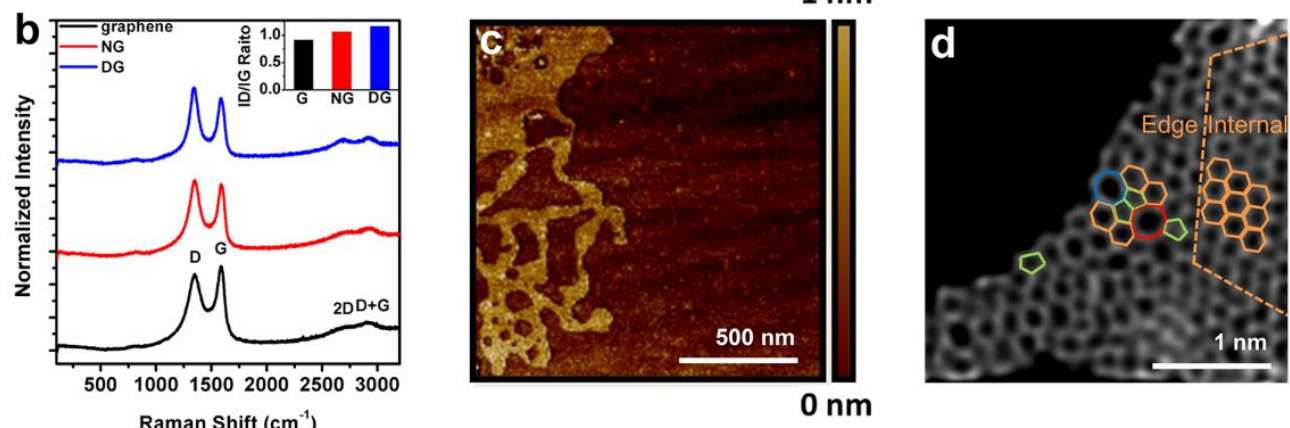

Raman Shift $\left(\mathrm{cm}^{-1}\right)$
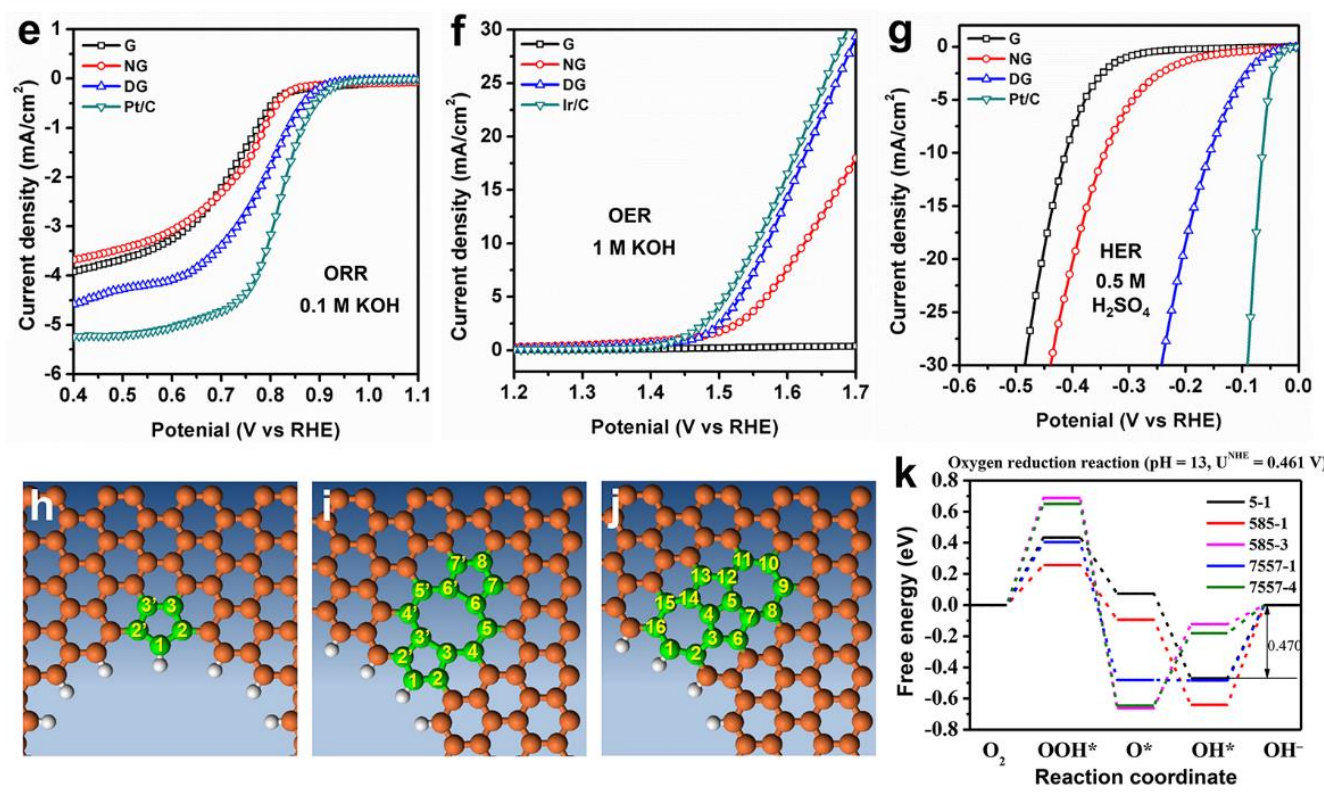

Fig. 12 (a) A schematic diagram showing the synthesis of DG; (b) Raman spectra of pristine graphene, NG, and DG; (c) An atomic force microscopy image of the DG; (d) High-angle annular dark-field (HAADF) image of DG. Hexagons, pentagons, heptagons, and octagons were labeled in orange, green, blue, and red, respectively; (e) ORR performance evaluations of the prepared samples under an oxygensaturated 0.1 M KOH solution; (f) OER activity of the prepared samples tested in $1 \mathrm{M} \mathrm{KOH}$; (g) HER performance of the prepared sample tested in $0.5 \mathrm{M} \mathrm{H}_{2} \mathrm{SO}_{4}$; (h) Edge pentagon; (i) 5-8-5 defect; (j) 755-7 defect; (k) Calculated energy profiles for the ORR pathway on defective graphene in an alkaline solution. ${ }^{36}$ Reprinted with permission from ref. 36. Copyright 2016, John Wiley and Sons.

Many additional investigations also support the proposed defects driven catalysis mechanism for the ORR. It is shown that the edges of the carbon materials play crucial roles for the electrocatalysis, since they are enriched with abundant defective sites for the reactions. ${ }^{108,}{ }^{188-191}$ Wang and co-workers 
used a micro apparatus studied the ORR behaviors of the edges and basal planes in a highly oriented pyrolytic graphite (HOPG). ${ }^{192}$ As shown in Fig. 13 (a), the HOPG is the working electrode, an airsaturated droplet is the electrolyte, a $\mathrm{Pt}$ wire is the counter electrode, and a $\mathrm{Ag} / \mathrm{AgCl}$ wire in a capillary tube connected with the droplet is the reference electrode. The ORR test with the droplet located at the edge (Fig. 13 (b)) and basal plane (Fig. 13 (c)) of the HOPG shows that the HOPG edge is much more active than that of the basal plane (Fig. 13 (d)). This result suggests the advantages of the edge defects for the electrocatalysis. The DFT calculations reveal that the higher charge densities of the edge carbon atoms contributed to the higher ORR activity of the HOPG edges. ${ }^{192}$ In addition, Peng et al. synthesized two kinds of CNTs with quite different percentages of edge carbon atoms. ${ }^{193}$ They found that the fishbone CNTs (F-CNTs) with as high as $31.7 \%$ edge carbons is much more active than that of the parallel CNTs (P-CNTs)) that only have 1.8\% edge carbons for the ORR. It is shown from the DFT simulations that compared to the basal planes, the edge carbon atoms could adsorb the oxygen molecules more easily. This is because the adjacent carbon atoms could carry more positive charge owing to the existence of the edge defective sites. Therefore, the charge re-distribution attributed to the enhanced ORR activity of the defective F-CNTs. ${ }^{16,193,194}$ Besides, Zhu et al. prepared a 3D web-like horizontally-aligned carbon nanotube-graphene (CNT-G) hybrid with rich edge sites. Compared with the N-doped graphene, the defective CNT-G is a better support to capture and disperse the Pt particles to promote the ORR. ${ }^{115}$
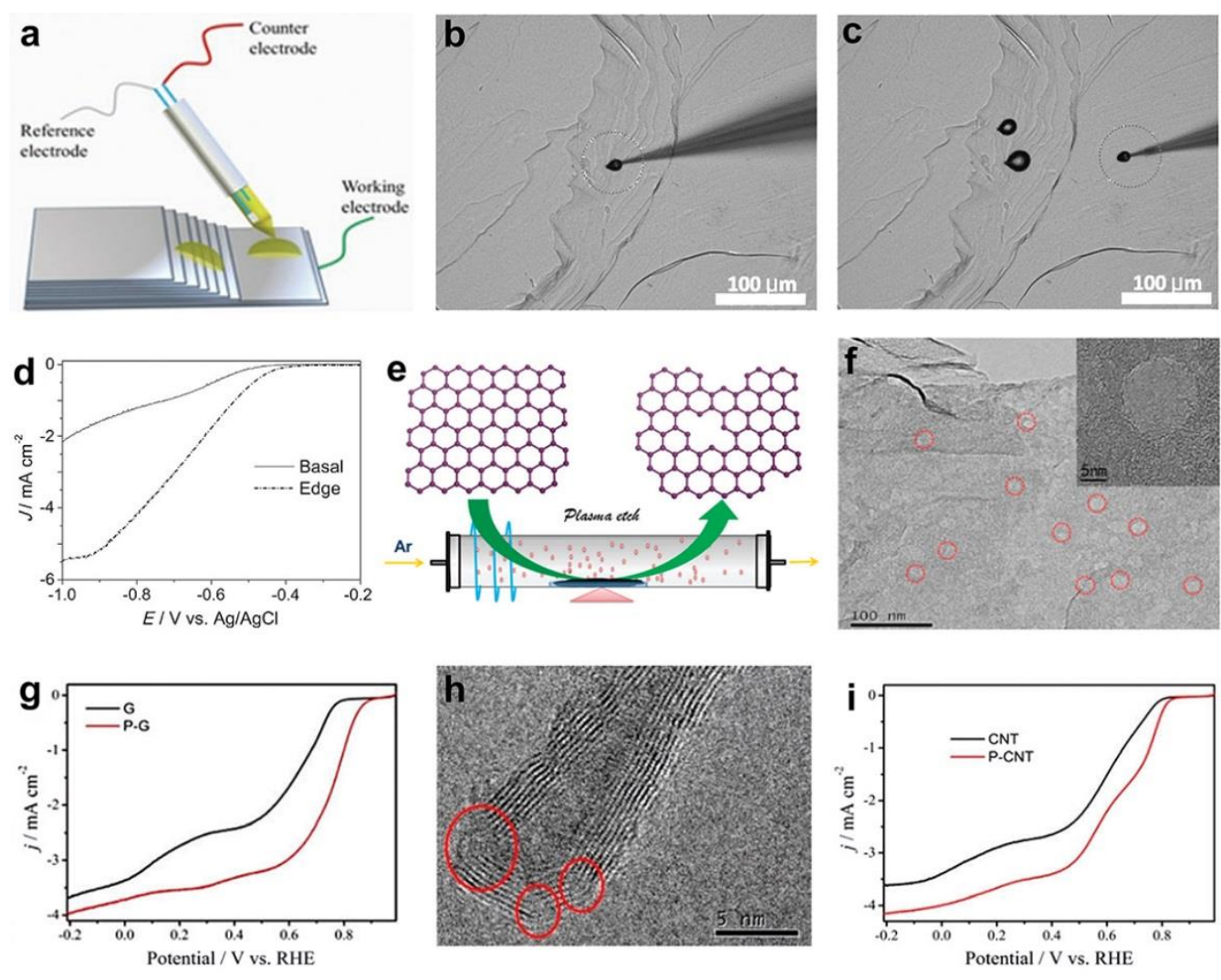

Fig. 13 (a) Micro apparatus for the ORR electrochemical experiment; (b) Optical photograph of the HOPG as the working electrode with the air-saturated droplet deposited on the edge of the HOPG; (c) 
The air saturated droplet was deposited on the basal plane of the HOPG electrode; (d) LSV curves of the ORR tested for a droplet located on different locations. ${ }^{192}$ Reprinted with permission from ref. 192. Copyright 2014, John Wiley and Sons. (e) Scheme of the preparation of the edge-rich and dopant-free graphene by Ar plasma etching; (f) HRTEM image of the plasma treated graphene; (g) LSV test results of $\mathrm{G}$ and $\mathrm{P}-\mathrm{G}$ in an $\mathrm{O}_{2}$-saturated $0.1 \mathrm{M} \mathrm{KOH}$ solution; (h) TEM image of the Ar plasma treated carbon nanotubes; (i) LSV curves of the plasma treated edge-rich carbon nanotubes tested in an $\mathrm{O}_{2}$-saturated 0.1 M aqueous KOH solution. ${ }^{195}$ Reproduced from Ref. 195 with permission from The Royal Society of Chemistry.

Apart from the removal of heteroatoms to create defects in the carbon matrix, another commonly used method is to bombard the target carbon materials by plasma irradiation. ${ }^{117,171,196-200}$ For example, Dai and Wang et al. utilized an argon plasma etching method (Fig. 13 (e)) produced edge/defect-rich graphene and CNTs with improved ORR performance. ${ }^{195}$ It can be observed from the high-resolution TEM images in Fig. 13 (f) and (h) that both the argon plasma-treated graphene (P-G) and CNT (P-CNT) maintained their original structures. Meanwhile, many nano-sized holes and edge defects can also be clearly found in the P-G and P-CNT, respectively. The defective nature of the P-G and P-CNT was further revealed by the corresponding Raman spectra. The ORR test results show that compared with the pristine graphene and CNT, both the P-G and P-CNT show obviously improved ORR activity (Fig. 13 (g) and (i)). ${ }^{195}$ These studies further support the universality of the defects promoted catalysis mechanism for the ORR in dopant-free carbon materials.

\subsection{Defects and Dopants Co-Promoted ORR}

For fabricating defective carbon materials through the post treatment methods, particularly with the assistance of the heteroatoms, it will unavoidably bring foreign atoms into the defective carbons. In other words, various defects and the residual heteroatoms (nitrogen is the most common one) are normally coexisted in the defective carbon materials. Therefore, some researches state that the heteroatoms in the defective carbons are also contributing to the electrocatalysis. For example, Zhang and co-workers studied the influence of the topological defects and the dopant nitrogen on the ORR in defective metal-free catalysts. ${ }^{66,201}$ As shown in Fig. 14 (a), a template method was used for synthesizing a N-doped graphene mesh (NGM) full of edges and topological defects. ${ }^{66}$ The TEM image of the NGM in Fig. 14 (b) shows the existence of nanosized holes over the plane. These holes were produced because the use of the MgO template. The corresponding Raman spectra in Fig. 14 (c) confirmed the defective nature of the NGM. Impressively, the defective NGM sample shows similar ORR activity to that of the $\mathrm{Pt} / \mathrm{C}$. It is revealed that both the nitrogen and the defective structures of the NGM account for its 
remarkable ORR activity. The DFT calculations reveal that the nitrogen doping-induced sites located at the graphene edges show much lower overpotentials than that of the plane. It is quite possible that the nitrogen further modified the electronic structures of surrounding edge carbon atoms, thus further promoted the ORR. In addition, different types of defects were considered in the DFT simulations (Fig. 14 (d)). It is revealed that the best configuration for the ORR is a type of five-carbon ring near the sevencarbon ring (C5+7), as shown in the volcano plot in Fig. 14 (e). ${ }^{66}$
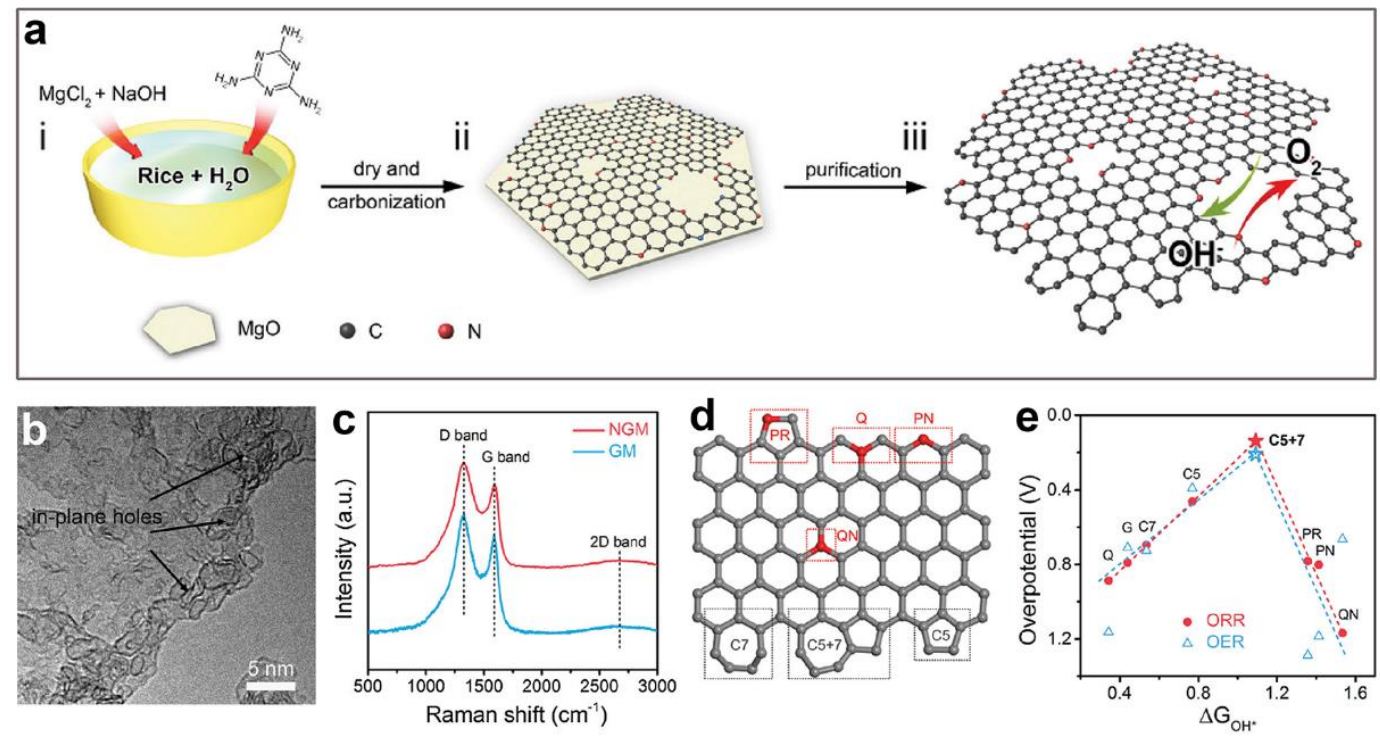

Fig. 14 (a) Schematic showing the preparation process of NGM catalyst; (b) High-resolution TEM image of the synthesized porous graphene; (c) Raman spectra of NGM and GM; (d) A schematic graphene nanoribbon with different kinds of $\mathrm{N}$-doping or topological defects, PR: pyrrolic nitrogen; PN: pyridinic nitrogen; Q: quaternary nitrogen on the edge; QN: quaternary nitrogen in the bulk phase; C5: five-carbon ring; C7: seven-carbon ring; $\mathrm{C} 5+7$ : five-carbon ring adjacent to seven-carbon ring; (e) ORR volcano plot (red line) of the overpotential versus the adsorption energy of $\mathrm{OH}^{*} .{ }^{66}$ Reprinted with permission from ref. 66. Copyright 2016, John Wiley and Sons.

Considering the heteroatoms could effectively tune the electronic structures of the carbon atoms, it is a feasible way of further improving the ORR performance of the defective carbons by introducing the heteroatoms. Following this principle, Zhao and co-worker used a facile thermal pyrolysis assisted method synthesized a N, B co-modified graphitic carbon nanocage (NB-CN) for multifunctional electrocatalysis (Fig. 15 (a)). ${ }^{202}$ The SEM and TEM images in Fig. 15 (b) to (f) show that the resulting defective carbons exhibit a hollow structure after leaching the $\mathrm{Co}_{2} \mathrm{P}$ core. Apparently, the edges of the NB-CN are enriched with defects, which is further confirmed by the corresponding Raman spectrum in Fig. $15(\mathrm{~g})$. The DFT calculations reveal that the dopant near the defective sites are more efficient for the trifunctional electrocatalysis (Fig. 15 (h)). This is consistent with the previous investigations that the 
heteroatoms are effective in tuning electronic configurations of the nearby carbon atoms to enhance their ORR activity. The remarkable ORR and OER performance of the NB-CN can be observed from Fig. 15 (i). The excellent electrocatalytic performance of the NB-CN render it as an ideal bifunctional catalyst for $\mathrm{Zn}$-air battery applications. ${ }^{202}$

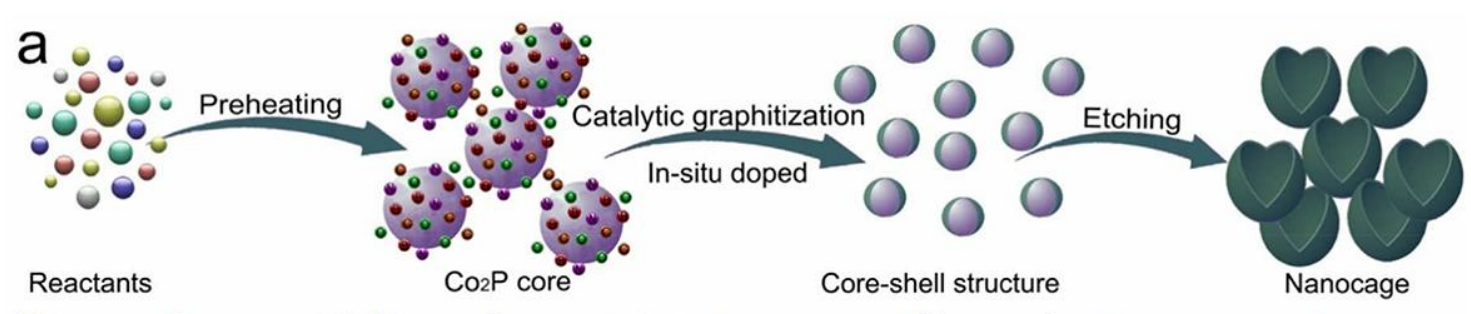

$\odot$ Chitosan $\odot \mathrm{Co}\left(\mathrm{CH}_{3} \mathrm{COO}\right)_{2} \odot \mathrm{Red} \mathrm{P} \odot \mathrm{H}_{3} \mathrm{BO}_{3} \odot \mathrm{KOH} \bullet$ Carbon atom $\bullet$ Oxygen atom $\bullet$ Nitrogen atom $\bullet$ Boron atom
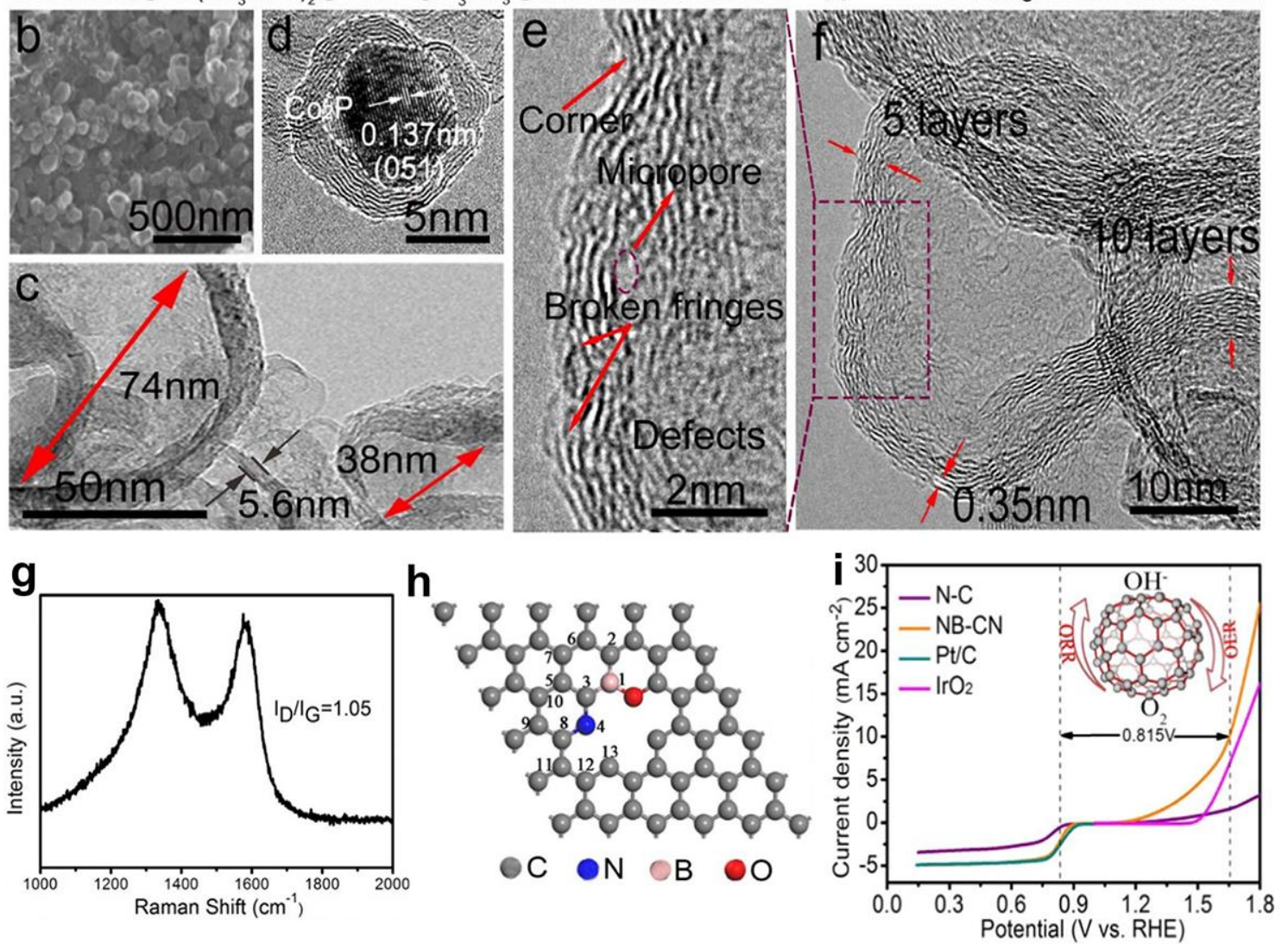

Fig. 15 (a) Illustration of the formation mechanism of graphitic carbon nanocage; (b) SEM image of NB-CN; (c) TEM image of NB-CN; (d) HR-TEM image of NB-CN before acid washing; (e, f) HRTEM images of NB-CN; (g) Raman spectrum of NB-CN; (h) Relative position of N, B and O for the N, B-codoped graphene considered in the calculations; (i) LSV curves of N-C, NB-CN, Pt/C and $\mathrm{IrO}_{2}$ catalyst on a RDE (1600 rpm) in $0.1 \mathrm{M} \mathrm{KOH}$ solution. ${ }^{202}$ Reprinted with permission from ref. 202. Copyright 2017, Elsevier.

\subsection{The Influence of Defect Density on Electrocatalysis}


The newly established defects promoted catalysis mechanism shows that certain types of topological carbon defects are the active sites for the electrocatalysis. Therefore, it is essential to reveal the relationship between the effective defects and the electrocatalytic performance of the defective carbons. For example, how the density of defects correlated with the electrocatalytic performance. To achieve the quantitatively analysis of the defects in a defective graphene, Ren et al. created different concentrations of defects on a single layer graphene via argon plasma irradiations. ${ }^{203}$ Both the advanced characterizations (such as the scanning electrochemical microscopy (SECM) and Raman mapping spectroscopy) and the theoretical calculations were used to probe how the electrocatalytic properties correlated with the defect density of the graphene. The results show that a moderate density of defects not only could favour the heterogeneous electron transfer (HET) rate, but also could maintain the good conductivity of the graphene sheet. In order to minimize the experimental variables, the same single layer graphene was used for creating different concentrations (by controlling the argon plasma irradiation time and ion dose) of defects on $100 \times 100 \mu \mathrm{m}^{2}$ squares. From the Raman spectra in Fig. 16 (a) and the corresponding D band mapping images of the defective graphene patterns in Fig. 16 (f) inset, it can be seen that pattern E shows the highest concentration of defects. Fig. 16 (b) shows that all the defective patterns exhibit higher feedback current than that of the pristine graphene. This result indicates the effective role of the newly produced defects for the electrochemical reactions. Besides, it also shows that different densities of defects can be targeted produced on the graphene by adjusting the argon plasma irradiation time and ion dose. It is shown in Fig. 16 (c) to (e) that the mean distance of defects is an important indicator in reflecting the concentration of defects. For example, the graphene can be fully activated without losing the structure integrity at a moderate defect density, but the HET rate constant achieves a peak value (Fig. 16 (f)). This is consistent with the testing results in Fig. 16 (g), suggesting that the optimal distance between defects is about $2 \mathrm{~nm} .{ }^{203}$ This study provides a method in measuring and calculating the density of defects in defective carbon materials. However, the precise control of the defects density and type is still a long way to go. These further achievements need the assistance of more advanced and well-designed experiments. 

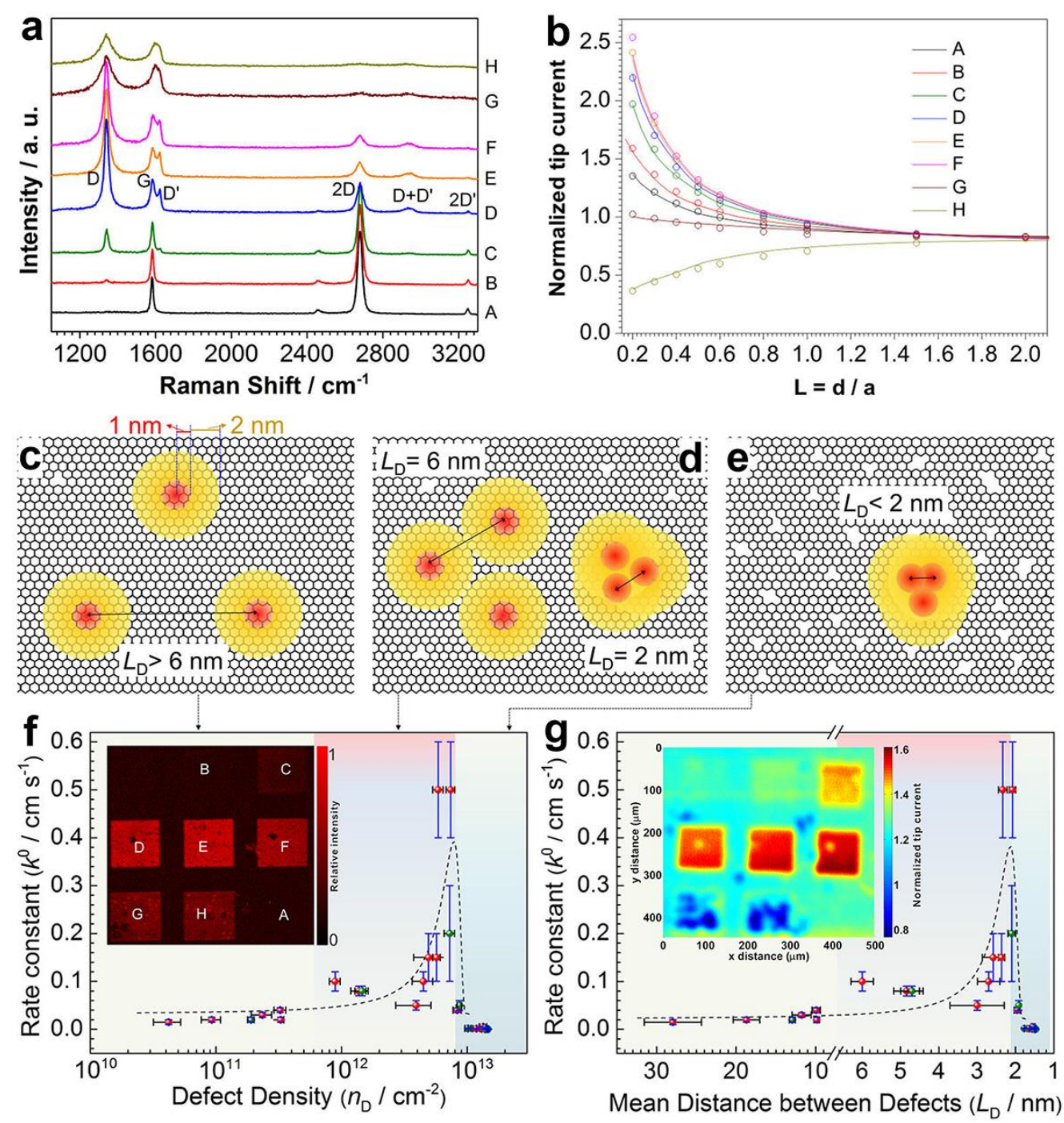

Fig. 16 (a) Raman spectra of the defective graphene patterns; (b) SECM approach curves obtained on each defective graphene pattern, with a tip potential of $0.4 \mathrm{~V}$ and a substrate potential of $0.18 \mathrm{~V}$; (c) to (e) The microscopic model in different defect density ranges: (c) Low defect density with $L_{\mathrm{D}}>6 \mathrm{~nm}$; (d) Moderate defect density with $2 \mathrm{~nm} \leq L_{\mathrm{D}} \leq 6 \mathrm{~nm}$; (e) High defect density with $L_{\mathrm{D}}<2 \mathrm{~nm}$. The area in red is the structurally disordered area with a radius of $1 \mathrm{~nm}$, and the area in yellow is the electronically activated but structurally preserved area $(1 \mathrm{~nm}<r<3 \mathrm{~nm})$; (f) The standard HET rate constant $k_{0}$ as a function of defect density $n_{\mathrm{D}}\left(\mathrm{cm}^{-2}\right)$, inset: Raman mapping of the $\mathrm{D}$ band of the defective graphene patterns; (g) The mean distance between defects $L_{\mathrm{D}}(\mathrm{nm})$, inset: SECM images of the same defective graphene patterns. The dashed lines in ( $\mathrm{f}$ ) and (g) are a guide for the eye only. ${ }^{203}$ Reprinted with permission from ref. 203. Copyright 2014, American Chemical Society.

\section{Coordination of Carbon Defects and Atomic Metal Species}


Metal based single atom catalysts (M-SACs) have been attracting extensive research interest due to their multiple advantages. For example, the maximum atom efficiency, the low utilization of precious metals, the controllable reaction selectivity, and the superb specific activity. Besides, the tuneable electronic structures of the M-SACs that owing to diverse metal and non-metal coordination motifs at atomic levels render them appropriate for a wide range of applications. ${ }^{204-206}$ So far, remarkable catalytic performance of these M-SACs has been demonstrated in CO oxidation ${ }^{207}$ and water-gas shift reaction. ${ }^{208}$ With respect to the design and synthesis of next generation M-SACs, continuous efforts have been focusing on metal oxide supported M-SACs due to the strong interactions between the atomic metal species and the metal oxide support. ${ }^{45,209,210}$ Meanwhile, defective carbon materials have recently been of particular interest as SAC hosts in electrocatalysis because of their advantageous features, including the large surface area, high electronic conductivity, chemical stability, and abundant defect sites for potential metal-support coordination. ${ }^{38,44}$

\subsection{Metal Atoms Induced Carbon-Defects Based Coordination Structures}

Theoretically, similar to the heteroatoms to tune the electronic structures of carbon materials, single atoms can also be regarded as foreign elements. Therefore, it can also modify the coordination environment of the carbon atoms. This could be the underlying reasons for the enhanced catalytic performance of the M-SA@carbon based electrocatalysts. The early study on M-SA@carbon based catalysts was introduced by Zhang and co-workers in 2011. ${ }^{211}$ They created a metal-vacancy coordination complex on the graphene by high energy atom/ion $\mathrm{Au}$ ) bombardment. Subsequently, the engraved vacancies were filled with the desired metallic dopants (Pt, Co and In) (Fig. 17 (a)). ${ }^{211}$ The created carbon defects ranging from single vacancies to holes of a few nanometers. This is because the laser ablation induced metal bombarding species has a wide distribution both in size and energy (Fig. 17 (b)). The vacancy structures were characterized using an aberration-corrected and monochromated TEM. Fig. 17 (c) shows the HRTEM images of the monovacancy (V1), bivacancy (V2), and trivacancy (V3), respectively. They are corresponding to the atomic models (Fig. 17 (d)) and the simulated HRTEM images (Fig. 17 (e)) for these three different types of vacancies. Hereafter, various approaches can be applied for trapping the desired metallic dopants using the prepared defective graphene. For example, Pt can be deposited by electron beam in a focused ion beam, while Co and In can be deposited by a conventional sputtering tool. The corresponding HRTEM images show that the atomic Pt species can be anchored by bivacancy or trivacancy coordination (Fig. 17 (f) to (k)). Furthermore, the DFT study reveals that the high binding energies of the Pt-vacancy complexes with different Pt-SA@carbon defects coordination structures can endow the complexes as stable active sites (Fig. 17 (1)). ${ }^{211}$ 

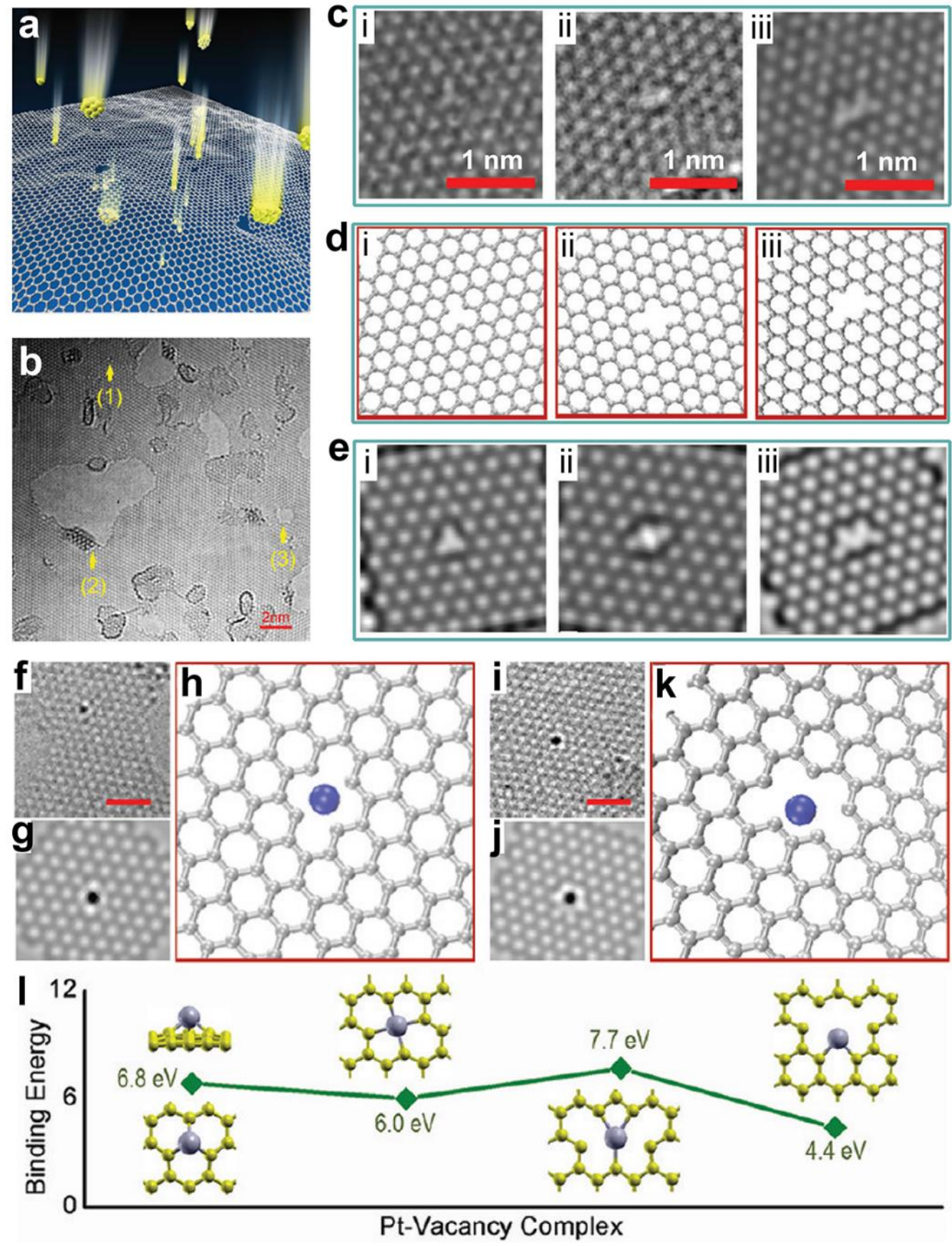

Fig. 17 (a) Schematic illustration of the high-energy particle bombardment graphene process; (b) HRTEM image of graphene after bombardment; (c) HRTEM images of (i) a monovacancy, (ii) a bivacancy, and (iii) a trivacancy; (d, e) Atomic models and simulated HRTEM images for the three different vacancy types in figure (c); HRTEM images of a Pt atom trapped in (f) a bivacancy and (i) a trivacancy. ( $h, k)$ Atomic models and $(\mathrm{g}, \mathrm{j})$ simulated HRTEM images for the Pt-vacancy complexes in (f, i). (l) Binding energies for different configurations. ${ }^{211}$ Reprinted with permission from ref. 211. Copyright 2012, American Chemical Society. 
Recently, Li and co-workers developed a novel strategy for fabricating SACs via creating M-N-C $(\mathrm{M}=\mathrm{Co}$ or $\mathrm{Fe})$ coordination structures at carbon defects using zinc imidazolate frameworks as precursors. ${ }^{40,212}$ It delivers samples with high loadings of atomic metal species, for example, up to 4 wt.\% for Co-N-C and 2 wt.\% for Fe-N-C, respectively. The basic concept of this method is relied on a pyrolysis process of the pre-designed bimetallic $\mathrm{Zn} / \mathrm{Co}$ metal-organic frameworks (MOFs) or $\mathrm{Zn}$ based MOFs capsulated Fe precursors. During the pyrolysis process, $\mathrm{Co} / \mathrm{Fe}$ can be reduced by the carbonized organic ligands and subsequently coordinated with the surrounding $\mathrm{N}$ atoms at the defect zones (Fig. 18 (a)). Remarkably, the chemical states of the atomically dispersed $\mathrm{Co} / \mathrm{Fe}$ species are highly related to the surrounding coordination environment (e.g., planar $\mathrm{Co}-\mathrm{N}_{4}-\mathrm{C}, \mathrm{Co}-\mathrm{N}_{2}-\mathrm{C}$, or Fe- $\mathrm{N}_{4}-\mathrm{C}$ ). The optimized chemical states of the formed complex will lead to the optimal electronic structures at the active sites for efficient ORR catalysis (Fig. 18 (b) to (f)). ${ }^{194}$ Furthermore, they extended this synthetic strategy to design defects induced dual-metal $(\mathrm{DM})-\mathrm{N}-\mathrm{C}(\mathrm{DM}=\mathrm{Co}$ and $\mathrm{Fe})$ coordinated sites in a $\mathrm{N}$-doped porous carbon (Fig. $18(\mathrm{~g})$ to $(\mathrm{k})$ ). The resulting $\mathrm{N}$-coordinated dual-metal catalyst $(\mathrm{Fe}, \mathrm{Co}) / \mathrm{N}-\mathrm{C}$ shows comparable ORR performance to the commercial Pt/C catalyst in an acidic electrolyte (half wave potential, $E_{1 / 2}: 0.863$ vs. $0.858 \mathrm{~V}$ ) (Fig. 18 (1)). ${ }^{213}$ The corresponding extended X-ray absorption fine structure (EXAFS) and X-ray absorption near-edge structure (XANES) characterizations combined with DFT calculations reveal that the co-existence of multiple coordination structures of the $(\mathrm{Fe}, \mathrm{Co}) / \mathrm{N}-\mathrm{C}$ at the whole defective zone may account for the excellent ORR performance (Fig. 18 (i) to (l)). ${ }^{213}$ 

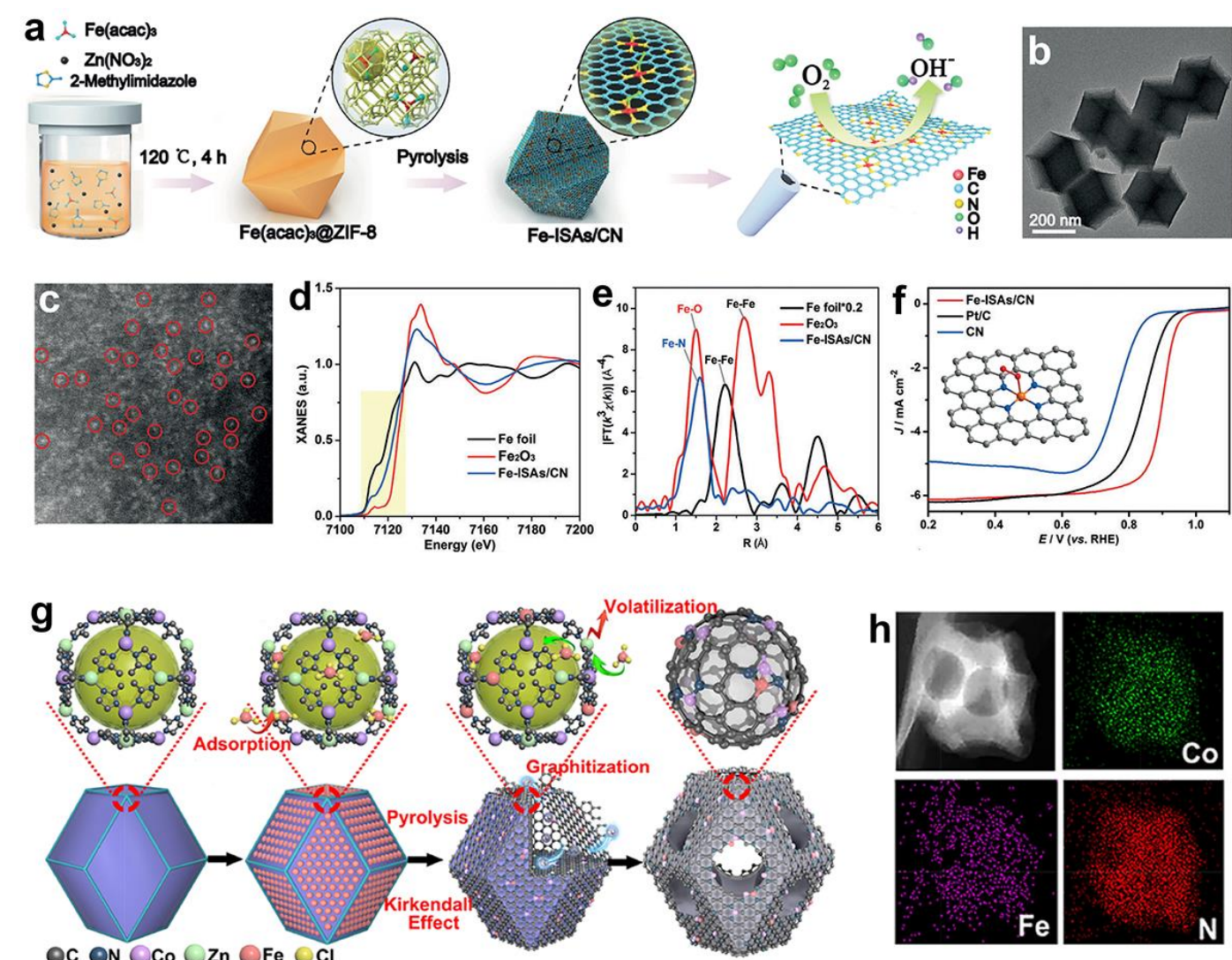

Oc ON $\odot$ co $\bigcirc \mathrm{Zn} \odot \mathrm{Fe} \odot \mathrm{Cl}$
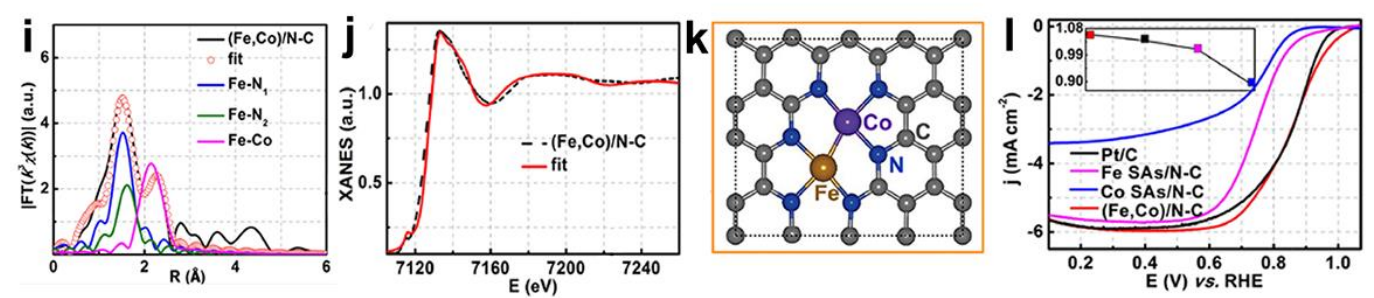

Fig. 18 (a) Schematic illustration of the formation of Fe-ISAs/CN; (b) TEM image of Fe-ISAs/CN; (c) HAADF-STEM image of the Fe-ISAs/CN. Single Fe atoms highlighted by red circles; (d) XANES spectra (the green area highlights the near-edge absorption energy); (e) Fourier transform (FT) of the Fe K-edge and wavelet transform (WT) of Fe-ISAs/CN; (f) ORR polarization curves in $\mathrm{O}_{2}$-saturated $0.1 \mathrm{~m}$ $\mathrm{KOH}$ solution, inset: schematic model of Fe-ISAs/CN, Fe (orange), N(blue), O(red), and C(gray). ${ }^{212}$ Reprinted with permission from ref. 212. Copyright 2017, John Wiley and Sons. (g) Preparation of (Fe,Co)/N-C; (h) Corresponding EELS mapping of Co, Fe, and N; (i) Corresponding Fe K-edge EXAFS fittings of $(\mathrm{Fe}, \mathrm{Co}) / \mathrm{N}-\mathrm{C}$; (j) Comparison between K-edge XANES experimental spectrum of $(\mathrm{Fe}, \mathrm{Co}) / \mathrm{N}-$ $\mathrm{C}$ (black dashed line) and theoretical spectrum calculated with depicted structure (solid red line); $(\mathrm{k})$ Proposed architectures of Fe-Co dual sites; (l) RDE polarization curves of Pt/C, Co SAs/N-C, Fe $\mathrm{SAs} / \mathrm{NC}$, and $(\mathrm{Fe}, \mathrm{Co}) / \mathrm{N}-\mathrm{C}$ in $\mathrm{O}_{2}$-saturated $0.1 \mathrm{M} \mathrm{HClO}_{4}$ solution. Inset: $E_{\text {onset }}$ of different catalysts. ${ }^{213}$ Reprinted with permission from ref. 213. Copyright 2017, American Chemical Society. 
More recently, the importance of defect coordination chemistry in oxygen-involving electrocatalysis has been continuously demonstrated and summarized by several groups. ${ }^{67,214-218}$ For example, the electronic state is asymmetrically tailored at the vacancy or reconstructed carbon defect sites of graphene. This modification not only could enhance the ORR performance, but also be functional for other electrocatalysis, such as the OER and HER. ${ }^{67,218}$ Besides the defects themselves could catalyze the electrochemical reactions (according to the proposed catalysis mechanism based on defect structures), defects are also capable of providing unique sites for trapping metallic species. ${ }^{9,113 \text {, }}$ ${ }^{219}$ Due to the different structures and sizes of the defects, one or more metal atoms might be trapped into the specific defect site.

Yao and co-workers systematically studied the electrocatalytic behaviors of the catalysts with defects in graphene coordinated with atomic Ni species. The catalysts were prepared through a facile impregnation method followed by an acid leaching process (Fig. 19 (a)). ${ }^{38}$ The corresponding structure characterizations by using the probe-corrected TEM and the XANES fittings show that three possible coordination structures of atomic Ni trapped in graphene defects (denoted as aNi@Di-vacancy, aNi@D5775 and aNi@Perfect) may coexist in the as-prepared A-Ni@DG catalyst (Fig. 19 (b) to (d)). Remarkably, the A-Ni@DG catalyst exhibits bifunctional activity for both the HER and OER with superb water splitting performance (an overpotential of $70 \mathrm{mV}$ for HER and $270 \mathrm{mV}$ for OER at a current density of $10 \mathrm{~mA} / \mathrm{cm}^{2}$ ). This performance is similar or even superior to those of the benchmark $\mathrm{Pt} / \mathrm{C}$ and $\mathrm{IrO}_{2}$ catalysts (Fig. 19 (e) and (f)). Furthermore, the DFT calculation results reveal that the electronic structures of the atomic Ni can be fine-tuned by bonding to diverse types of graphene defects. The different coordination of atomic Ni with defects in graphene is responsible for different reactions. For example, the presence of aNi@D5775 favors the HER, while the aNi@Di-vacancy exhibits optimal binding energies for $* \mathrm{O}$ and $* \mathrm{OOH}$ intermediates, thus being beneficial for the OER (Fig. 19 (g) to $(\mathrm{k}))^{38}$ 

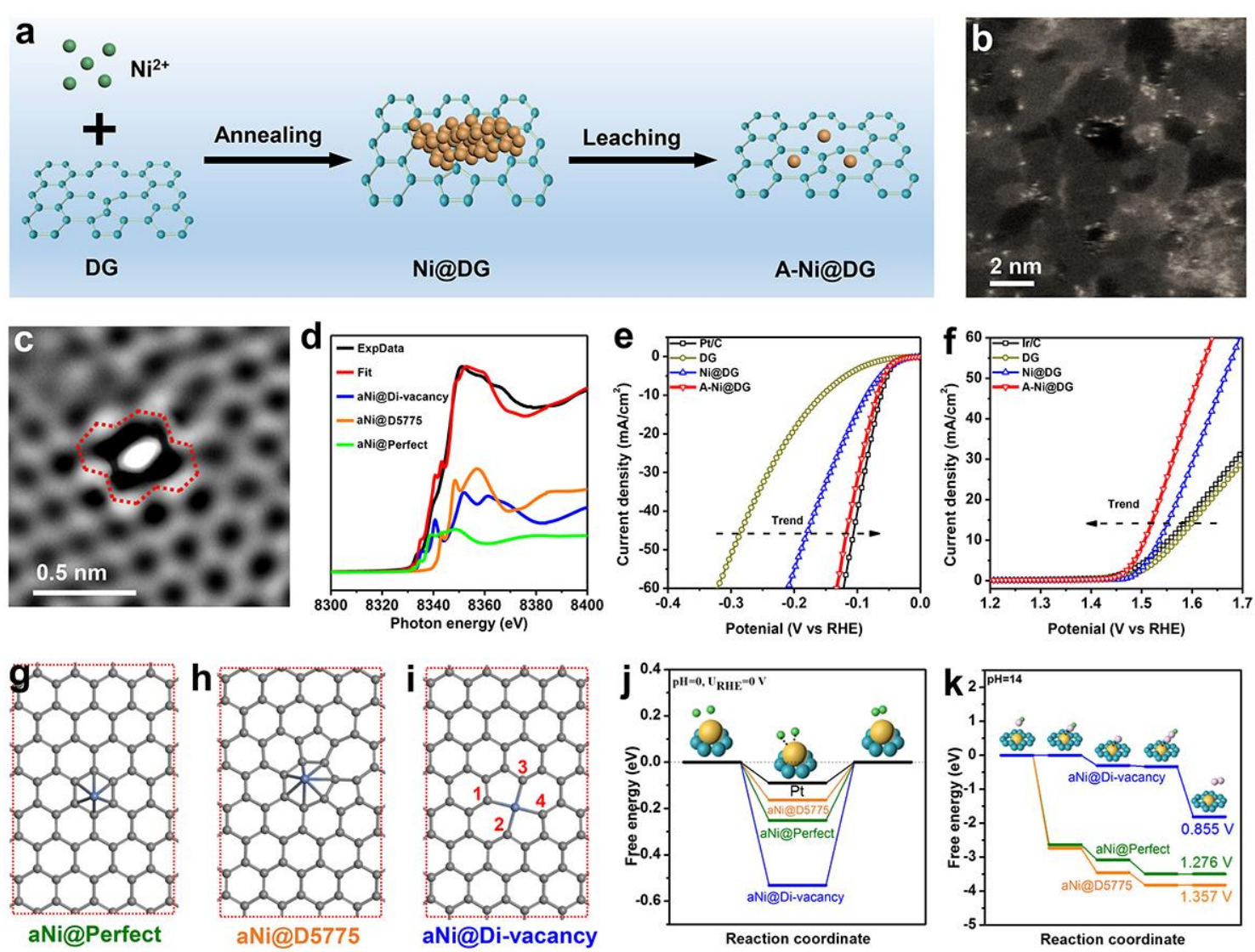
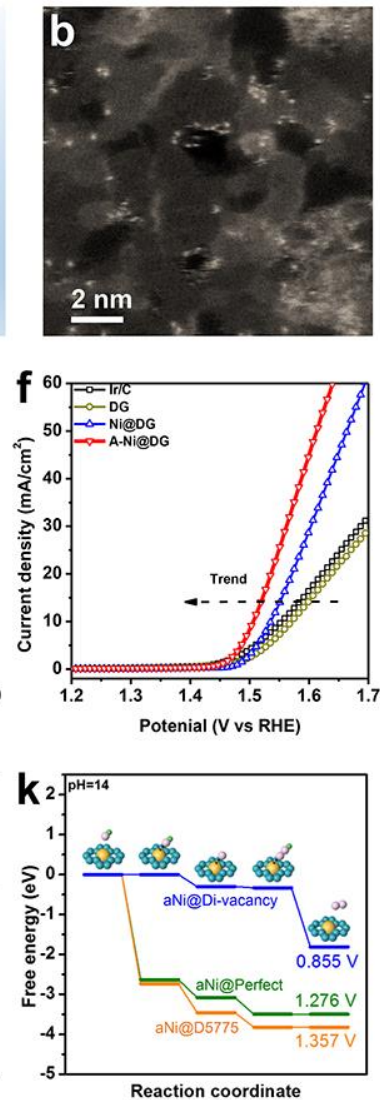

Fig. 19 (a) The fabrication schematic of A-Ni@DG; (b) HADDF-STEM image of A-Ni@DG; (c) The atomic resolution of the Di-vacancy; (d) The LCF analysis of XANES theoretical modelling. The red line is the superposition of the theoretical model simulations of the three configurations and fits the experimental data well; (e) HER polarization curves of DG, Ni@DG, A-Ni@DG, and Pt/C performed in $0.5 \mathrm{M} \mathrm{H}_{2} \mathrm{SO}_{4}$ electrolyte; (f) OER polarization curves of DG, Ni@DG, A-Ni@DG, and Ir/C performed in $1 \mathrm{M} \mathrm{KOH}$ electrolyte; (g-i) Illustrations of three different types of catalytic active sites corresponding to a single Ni atom supported on (g) perfect hexagons, (h) D5775, and (i) Di-vacancy; (j and k) Energy profiles of the three configurations (g-i) for HER (j) and OER (k), respectively. ${ }^{38}$ Reprinted with permission from ref. 38. Copyright 2018, Elsevier.

Currently, the well-defined metal-defect coordination structures could allow the in-depth understanding on the catalytic reaction pathways and rational design of the targeted catalysts with tailored activity. However, it is still quite challenging to prepare the targeted catalysts with the exclusive metal-defect coordination structures at atomic scales. To this end, Duan and co-workers reported a general hydrothermal-annealing method for synthesizing a series of monodispersed atomic transition metals $(\mathrm{M}=\mathrm{Fe}, \mathrm{Co}, \mathrm{Ni})$ trapped in a nitrogen-doped defective graphene with a definitive $\mathrm{M}-\mathrm{N}_{4}-\mathrm{C}_{4}$ moiety (Fig. 20). This will allow the correlation study on the unambiguous structures of the active sites and the specific electrochemical reactions. ${ }^{220}$ Apart from carbon defects containing $\mathrm{N}$ atoms, defective 
graphene with $\mathrm{S}$ can also trap atomically dispersed metal species. It is shown that the $\mathrm{S}$ coordinated metal species exhibit even more vigorous affinity to metal centres owing to the stronger Lewis base of $\mathrm{S}$ compared to $\mathrm{N}^{221,222}$ Besides, electrochemical activation method is also effective in fabricating defects coordinated single metals species (such as $\mathrm{Ni}$ ). ${ }^{28}$
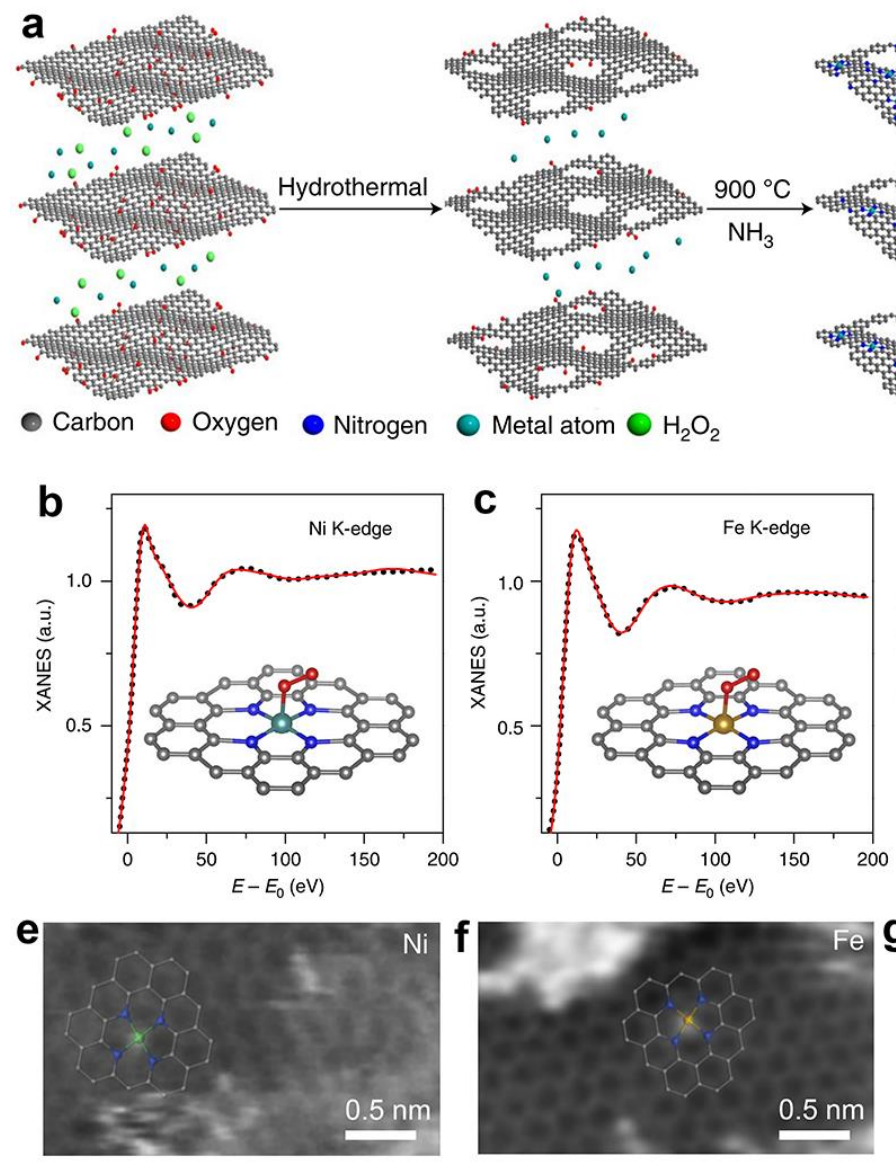
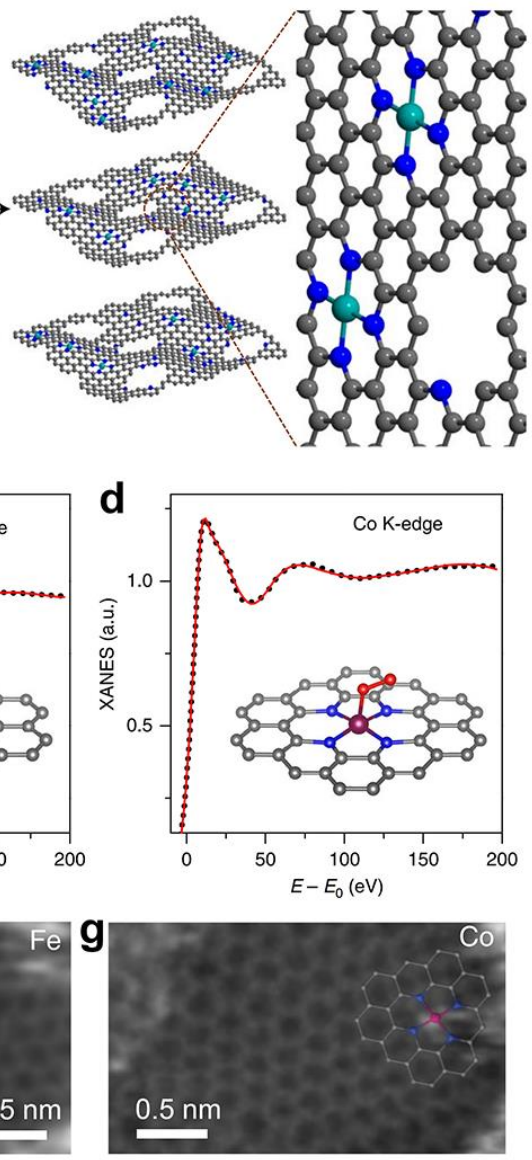

Fig. 20 (a) The preparation route to atomic $3 d$ metals embedded in nitrogen-doped holey graphene frameworks (M-NHGFs); (b) to (d) Comparison between the experimental XANES spectra (black dotted lines) for M-NHGFs and the best-fit theoretical spectra (solid red lines). The insets show the geometrically refined MN4C4 structure; (e) to (g) High-resolution TEM images enable the direct visualization of the atomic metals of $\mathrm{Ni}(\mathrm{e}), \mathrm{Fe}(\mathrm{f})$ and $\mathrm{Co}(\mathrm{g})$ embedded in the 2D graphene lattice. The overlaid schematics represent the structural models determined from XAFS analysis. ${ }^{220}$ Reprinted with permission from ref. 220. Copyright 2018, Springer Nature.

\subsection{Defect Engineering in Designing Catalytic Interfaces and Mass Production}

Interface catalysis is another important aspect in boosting the electrocatalysis. This is because the interactions at the interface between two or more materials will alter their electronic states and chemical properties. ${ }^{223,}{ }^{224}$ Generally, the principles of constructing a catalytic interface is governed by the 
following rules: (i) The complementary properties of the component materials for the targeted electrocatalysis; (ii) Sufficient anchor sites exist in either material for facilitating the formation of robust heterostructures; (iii) The designed hybrids should possess maximum catalytic interfaces for the rapid electron transfer to the reaction intermediates. Following these guidelines, Yao et al. designed and fabricated a heterostructured NiFe LDH-NS@DG10 hybrid by coupling of the exfoliated Ni-Fe layered double hydroxide (LDH) nanosheet (NS) with a defective graphene (DG) for overall water splitting (Fig. 21 (a)). ${ }^{225}$ Fig. 21 (b) shows that the NiFe LDH-NS are uniformly distributed on the DG support. It can be seen from Fig. 21 (c) that the overall water splitting performance of the NiFe LDH-NS@DG10 is better than that of the NiFe LDH-NS@graphene (NG and G). Impressively, compared to the reported non-precious metal electrocatalysts, the NiFe LDH-NS@DG10 shows the best performance for alkaline overall water splitting (Fig. 21 (d)). The DFT simulations further confirm that the formation of the heterostructure is crucial to the charge separation and redistribution on the hybrid (Fig. 21 (e)). Therefore, the newly form heterostructure in the complex promoted the OER and HER. ${ }^{225}$
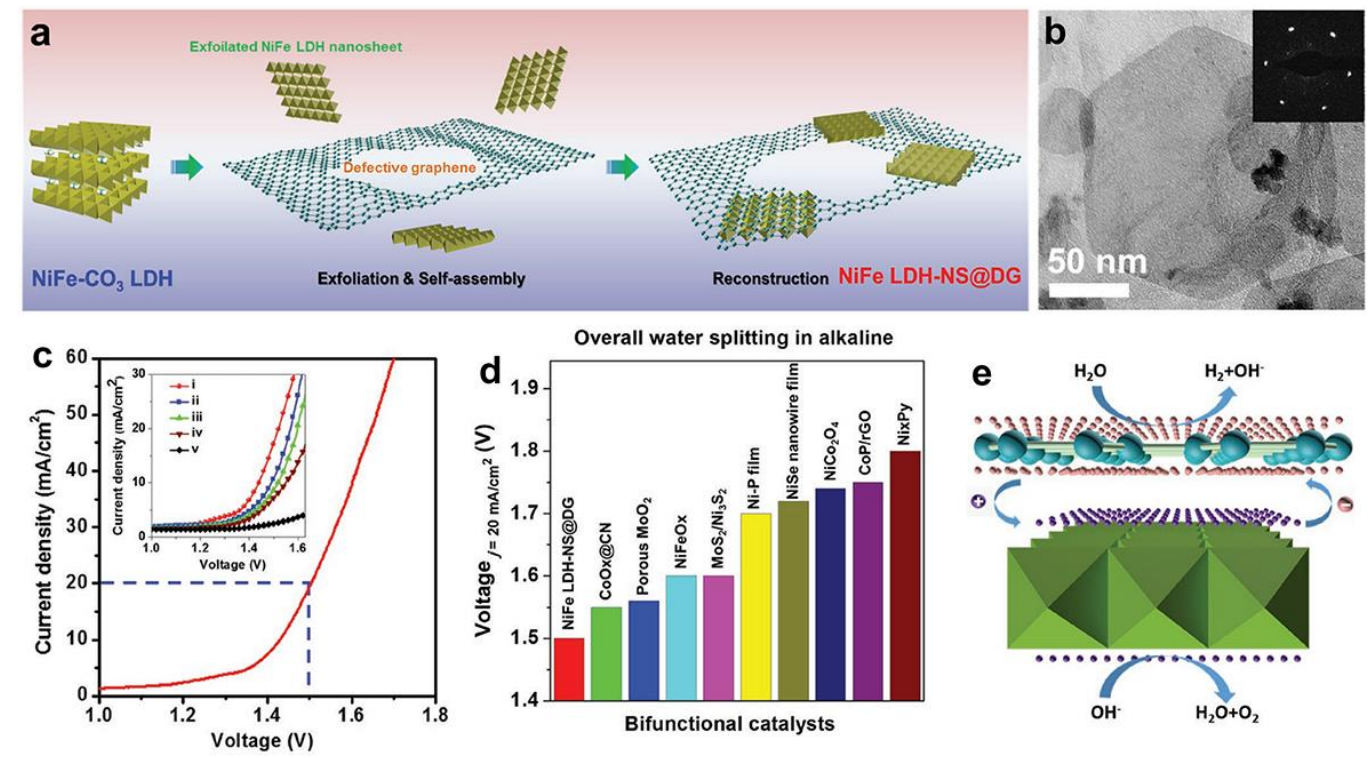

Fig. 21 (a) Schematic illustration of the preparation of NiFe LDH-NS@DG nanocomposite; (b) A typical exfoliated LDH-NS anchored on DG with corresponding selected area diffraction pattern (inset); (c) LSV curves of NiFe LDH-NS@DG10 as OER and HER bifunctional catalyst in $1 \mathrm{M} \mathrm{KOH}$ for overall water splitting, with the inset showing the different catalysts: (i) NiFe LDH-NS@ DG10 with $2 \mathrm{mg} \mathrm{cm}^{-2}$ loading, (ii) NiFe LDH-NS@DG10 with 1 mg cm${ }^{-2}$ loading, (iii) NiFe LDH-NS@NG10 with 2 mg cm ${ }^{-2}$ loading, (iv) NiFe LDH-NS @ G10 with $2 \mathrm{mg} \mathrm{cm}^{-2}$ loading, (v) bare Ni foam electrode; (d) Comparison of the required voltage at a current density of $20 \mathrm{~mA} \mathrm{~cm}{ }^{-2}$ for the NiFe LDH-NS@DG catalyst with other state-of-the-art noble metal free bifunctional catalysts; (e) The schematic of the probable electrocatalytic mechanism of Ni-Fe LDH-NS@DG for HER and OER is presented based on the DFT calculation results. The pink and purple spheres represent electrons and holes, respectively. ${ }^{225}$ Reprinted with permission from ref. 225. Copyright 2017, John Wiley and Sons. 
Similar to the utilization of the defect engineering knowledge in 2-dimensional materials (e.g., graphene) for designing the catalytic reaction interfaces, it is also crucial to use these techniques in 3dimensional materials (e.g., porous carbons). The 3-dimensional materials are prominent for the electrocatalysis because their robust porous structures could provide abundant active sites for the reaction, and sufficient channels for mass transportation, particularly after proper defect engineering. For example, Lou and co-workers reported a dynamic reaction method for the traction and stabilization of the isolated $\mathrm{Pt}$ species in a porous carbon matrix (PCM). The catalysts were prepared via the move of the atomic Pt species from the surface of the porous carbon sphere into the internal of the PCM (Fig. 22 (a) to (c)). This approach could significantly increase the density of the active sites. ${ }^{44}$ The shorter radial distance of Pt@PCM in the wavelet transform (WT) analysis (Fig. 22 (d)) and the higher white line intensity of Pt@PCM in the XANES profile (Fig. 22 (e)) confirm the successful loading of isolated $\mathrm{Pt}$ atoms into the carbon matrix by forming the $\mathrm{Pt}-\mathrm{N}-\mathrm{C}$ coordination structures at the defect zones (Fig. $22(\mathrm{~g}))$. The DFT results further reveal that the confined Pt atom at the defect vacancy with surrounding $\mathrm{C} / \mathrm{N}$ atoms as a whole should be the active site for the HER. This is because the catalytic effect decreases with an increase in the number of shells (Fig. 22 (f) to (h)). ${ }^{44}$ Particularly, the incorporated Pt single atoms may tune the electronic structures of the adjunct carbon atoms and make them active for the HER.
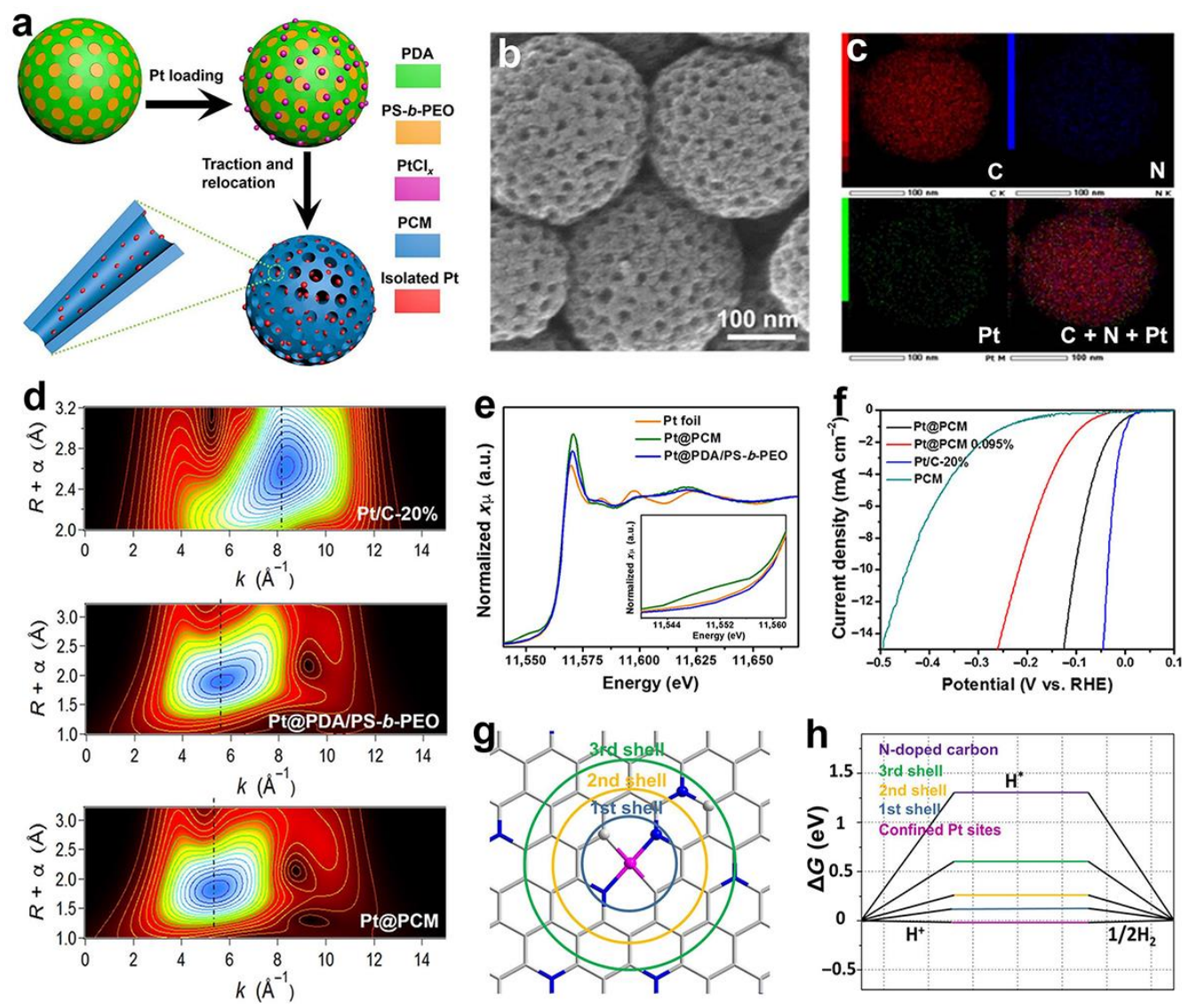

Fig. 22 (a) Schematic illustration of the synthetic procedure of Pt@ PCM; (b) SEM image of Pt@ PCM; (c) Element mapping images of C, N, and Pt with their overlapping image of Pt@PCM; (d) Wavelet 
transform (WT) for the $k^{3}$-weighted EXAFS signal; (e) Pt K-edge XANES experimental spectra with enlarged profile as the inset. a.u., arbitrary units; (f) LSV curves of various catalysts in $0.5 \mathrm{M} \mathrm{H}_{2} \mathrm{SO}_{4}$; (g) Schematic description for the coordination shells for the isolated Pt over the graphene; (h) $\Delta G_{\mathrm{H}^{*}}$ on pure and Pt-decorated graphene in different coordination shells. ${ }^{44}$ Reprinted with permission from ref. 44. Copyright 2018, The Authors, some rights reserved; exclusive licensee AAAS.

To realize the proof of "defect" concept for the mass production of efficient and cost-effective electrocatalyts, Yao and co-workers developed a controllable and scalable synthetic strategy by using defective carbons from cheap and earth-abundant resources (Fig. 23). For example, defective activated carbon based catalysts, ${ }^{9,63,219}$ seaweed derived carbon based materials, ${ }^{226-228}$ and macadamia nut shell derived carbon based electrocatalysts. ${ }^{26}$ The above research results addressed a very important issue that activated carbons can be highly active for electrocatalysis by applying the newly established defects promoted catalysis mechanism. This breaks a common sense that activated carbons were non-active for the electrocatalysis. This significant discovery forms the basis of the "defect" research for the mass production of electrocatalysts, because all carbons derived from biomass are activated carbons. More importantly, the method used to convert the biomass to carbons is controllable, scalable and very simple and cost-effective. All these advantages are crucial to the commercialization of the electrocatalysts, particularly for the widespread application of fuel cells. 

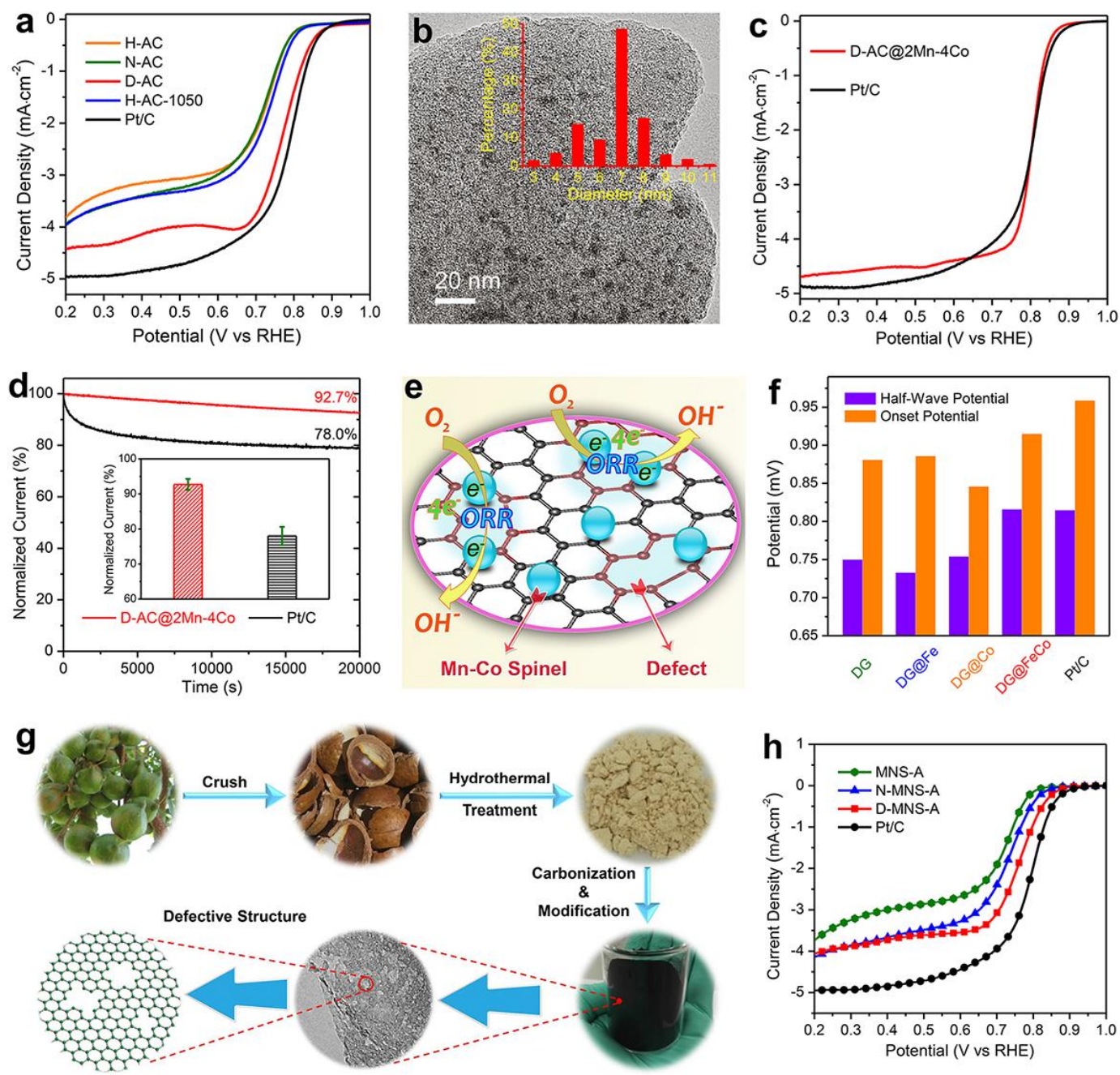

Fig. 23 (a) Linear sweep voltammetry (LSV) curves of the H-AC, N-AC, D-AC, H-AC-1050 and Pt/C measured at the rotation speed of $1600 \mathrm{rpm}$ in an $\mathrm{O}_{2}$-saturated $0.1 \mathrm{M} \mathrm{KOH}$ electrolyte. ${ }^{63}$ Reprinted with permission from ref. 63. Copyright 2016, The Royal Society of Chemistry. (b) TEM image of the DAC@2Mn-4Co (Inset: a histogram shows the particle size distribution); (c) ORR performance comparison between the D-AC@2Mn-4Co and the commercial Pt/C in $0.1 \mathrm{M} \mathrm{KOH}$ solution; (d) Amperometric i - t curves of the D-AC@2Mn-4Co and the Pt/C tested at $1000 \mathrm{rpm}$ in $\mathrm{O}_{2}$-saturated 0.1 $\mathrm{M} \mathrm{KOH}$ solution, the standard-deviation method was used to evaluate the resulting data; (e) Schematic representation showing the synergetic coupling effects between the unique defects in the D-AC and the introduced Mn-Co spinel promoted ORR. ${ }^{219}$ Reprinted with permission from ref. 219. Copyright 2016, John Wiley and Sons. (f) Half-wave potential and onset potential comparisons of the synthesized defective based samples and commercial Pt/C; ${ }^{113}$ Reprinted with permission from ref. 113. Copyright 2017, The Royal Society of Chemistry. (g) Procedures to prepare defective porous carbons from macadamia nut shell waste; (h) ORR performance of the prepared samples and $\mathrm{Pt} / \mathrm{C}$ measured at the rotation speed of $1600 \mathrm{rpm}$ in an $\mathrm{O}_{2}$-saturated $0.1 \mathrm{M} \mathrm{KOH}$ solution. ${ }^{26}$ Reprinted with permission from ref. 26. Copyright 2018, John Wiley and Sons. 


\section{Outlook and Conclusions}

In recent years, although remarkable achievements have been reached in the field of electrocatalysis, more in-depth and systematic work, both theoretically and experimentally, should be continuingly carried out to tackle the tricky issues regarding the defects promoted electrocatalysis. This study will be beneficial for the design and synthesis of electrocatalysts with the expected performance for different applications. The future development of the defective carbon area should be focused on four major directions, as shown in Figure 24 and specified below.

(i) Controllable synthesis of various defective carbon materials. Currently, it is still difficult to produce single type of defect in the carbon materials, such as the pentagon and G585 defects. Therefore, how to create the desired effective defects in different carbon materials is worth in-depth investigations. It provides a feasible approach to prepare low cost but highly active metal-free electrocatalysts. Besides, for the fabrication of defective carbons coordinated single metal species, it is a promising way of increasing the density of the active sites by loading more metal species. For example, using 3dimensional highly defective porous carbon materials to trap more single atoms.

(ii) Advanced characterizations of carbon defects and defects coordinated single metal species. It is an important direction of research to observe the detailed structures of various defects and defects captured single metal species by using advanced TEM technologies. Besides, the Raman mapping and positron annihilation spectroscopy techniques are effective in characterizing the defect density and the type of defect, respectively. These advanced characterizations will provide strong experimental evidences to understand the origin of the ORR. Hence, it will be helpful for the design of efficient defective carbon based electrocatalysts for practical applications.

(iii) Reveal the reaction mechanisms of defects promoted electrocatalysis. At present, the theoretical simulations are normally applied to assist the understanding of the pathways of the electrochemical reactions. However, the in situ investigations on the reaction intermediate products are still very difficult. This is because the reaction processes involve solid-liquid-gas three phrases and extremely complex. It is therefore crucial to conduct well-designed experiment to probe the reaction intermediates directly. The experimental results combined with the theoretical calculations could help to gain comprehensive and thorough understandings on the reaction mechanisms. This will lead to the rational and controllable design and fabrication of active electrocatalysts for various catalytic reactions.

(iv) Accelerate the development of defective carbon based electrocatalysts for practical applications. The current defective carbons could effectively catalyze the ORR in alkaline electrolytes, but not in acidic solutions. Great effort should be devoted to exploiting defective carbons for catalyzing the ORR in acidic media, since most of the practical fuel cells are using acidic electrolytes to eliminate the effect of carbon dioxide. Due to the intrinsic size flexibility of fuel cells, they can be used in both portable and 
stationary power generation systems. A case in point is the fuel cell powered vehicles. Therefore, design and synthesize more effective and economic acidic ORR electrocatalysts to speed up the practical applications of fuel cell powered products is of great significance.
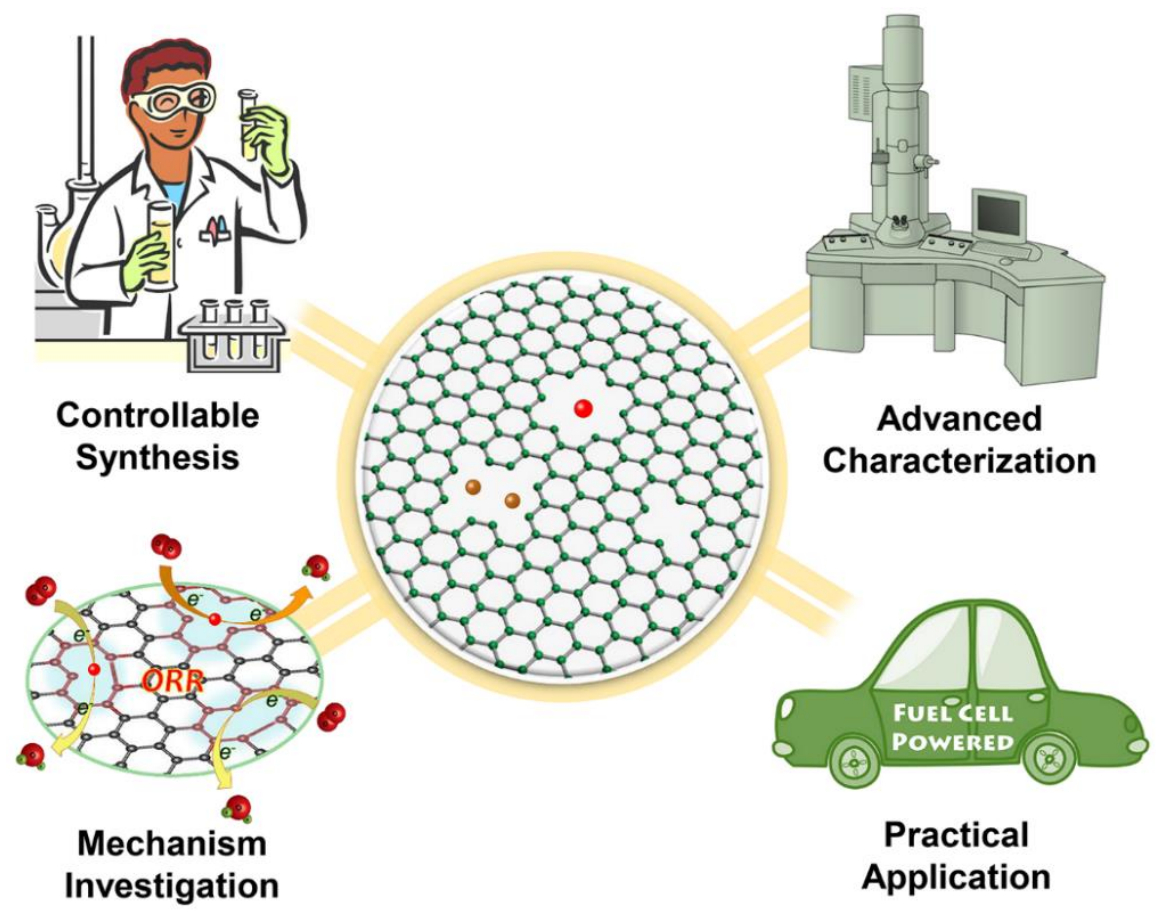

Fig. 24 Future development directions of the defective carbon field. The TEM photo courtesy of Togo picture gallery, the cartoon car photo courtesy of Free Stock Photo.

In summary, defective carbon materials are the promising candidates to substitute the rare and expensive Pt-based ORR catalysts owing to their high activity and low cost. Therefore, it has aroused intensive research interest in recent years. This review highlighted recent development on defective carbons promoted ORR from three general aspects: (i) Non-metal induced defective carbons for the ORR. The understanding of the active sites of various heteroatoms modified carbon materials, such as carbon nanotubes, graphene and mesoporous carbons is now becoming clear. This is because the newly established defects promoted catalysis mechanism could reasonably explain the ORR performance improvement. It is revealed that the actual ORR active sites are the modified carbon atoms near the dopants, so the influence of the heteroatoms on the surrounding carbon atoms is decisive to the ORR performance of the modified carbon materials. (ii) Intrinsic carbon defects promoted ORR. As discussed, heteroatoms doping provides a method to make carbon materials active for the electrocatalysis, since it could change the electronic structures of the nearby carbon atoms. Therefore, any other methods that could alter the electronic environment of carbon atoms are also capable of enhancing the ORR performance. For example, the facile heteroatoms (nitrogen, sulphur, zinc, etc.) doping and removal, particle (electrons or ions) irradiation, and plasma treatment are the commonly used methods. Related 
examples on producing effective defects for the ORR are summarized in Section 4. Particularly, the proposed active defects for the ORR have been observed experimentally in the defective graphene with the assistance of the aberration-corrected high-resolution TEM, such as the edge pentagons and G585 defect. These experimental results combined with the corresponding theoretical simulations provided solid evidences to support the proposed catalysis mechanism based on defect structures. In addition, the defective graphene is also active for the OER and HER catalysis, showing the versatility of the defects driven catalysis mechanism for electrocatalysis. (iii) Coordination of carbon defects with atomic metal species. As a rising field, single atom catalysis has become a hot area of research in recent years. Fundamentally, single atoms are similar to that of heteroatoms doping to tune the electronic structures of carbon atoms in enhancing the electrocatalytic performance of the catalysts. In Section 5, we summarized recent work on single metal atoms coordinated carbon materials for efficient electrocatalysis. The configurations and coordination of the single atoms with the carbon/heteroatoms are discussed. These results will be beneficial for designing and fabricating target electrocatalysts for practical applications. Besides, the frequently used methods to prepare single metal atoms coordinated carbons are presented as well. For the mass production of fuel cells, the scalability and cost of the electrocatalysts are the crucial issues. In this regard, we introduced feasible ways of preparing active and low-cost electrocatalyts from activated carbon and biomasses. At last, the future development directions of the defective carbon filed was outlined to show its broad prospects. This review systematical summarized the representive and important work on defects carbon materials catalyzed ORR, which will be helpful for gaining an overall understanding on the advancement of this thriving area.

\section{Conflicts of interest}

There are no conflicts to declare.

\section{Acknowledgements}

The authors would like to thank Australian Research Council (ARC) for financial support. 


\section{Notes and references}

1. R. F. Service, Science, 2007, 315, 172.

2. J. Zhang, 2008, PEM Fuel Cell Electrocatalysts and Catalyst Layers. Springer-Verlag London

3. J. K. Nørskov, J. Rossmeisl, A. Logadottir, L. Lindqvist, J. R. Kitchin, T. Bligaard and H. Jónsson, J. Phys. Chem. B, 2004, 108, 17886-17892.

4. B. C. H. Steele and A. Heinzel, Nature, 2001, 414, 345-352.

5. D. Zhang, F. Wu, M. Peng, X. Wang, D. Xia and G. Guo, J. Am. Chem. Soc., 2015, 137, 62636269.

6. Y. Nie, L. Li and Z. Wei, Chem. Soc. Rev., 2015, 44, 2168-2201.

7. V. Tripkovic, I. Cerri, T. Bligaard and J. Rossmeisl, Catal. Lett., 2014, 144, 380-388.

8. L. Zhang, R. Iyyamperumal, D. F. Yancey, R. M. Crooks and G. Henkelman, ACS Nano, 2013, 7, 9168-9172.

9. X. Yan, Y. Jia, L. Zhang and X. Yao, Chin. J. Catal., 2017, 38, 1011-1020.

10. H. Yang, Y. Zhang, F. Hu and Q. Wang, Nano Lett., 2015, 15, 7616-7620.

11. K. Strickland, E. Miner, Q. Jia, U. Tylus, N. Ramaswamy, W. Liang, M.-T. Sougrati, F. Jaouen and S. Mukerjee, Nat. Commun., 2015, 6, 7343.

12. W. Ding, L. Li, K. Xiong, Y. Wang, W. Li, Y. Nie, S. Chen, X. Qi and Z. Wei, J. Am. Chem. Soc., 2015, 137, 5414-5420.

13. G. Wu, K. L. More, C. M. Johnston and P. Zelenay, Science, 2011, 332, 443-447.

14. Y. Liang, Y. Li, H. Wang, J. Zhou, J. Wang, T. Regier and H. Dai, Nat. Mater., 2011, 10, 780-786.

15. T. Odedairo, X. Yan, J. Ma, Y. Jiao, X. Yao, A. Du and Z. Zhu, ACS Appl. Mater. Interfaces, 2015, 7, 21373-21380.

16. K. Gong, F. Du, Z. Xia, M. Durstock and L. Dai, Science, 2009, 323, 760-764.

17. X. Zhao, H. Zhao, T. Zhang, X. Yan, Y. Yuan, H. Zhang, H. Zhao, D. Zhang, G. Zhu and X. Yao, J. Mater. Chem. A, 2014, 2, 11666-11671.

18. K. Ai, Y. Liu, C. Ruan, L. Lu and G. Lu, Adv. Mater., 2013, 25, 998-1003.

19. P. Chen, T.-Y. Xiao, Y.-H. Qian, S.-S. Li and S.-H. Yu, Adv. Mater., 2013, 25, 3192-3196.

20. Z.-S. Wu, S. Yang, Y. Sun, K. Parvez, X. Feng and K. Müllen, J. Am. Chem. Soc., 2012, 134, 90829085.

21. Y.-J. Wang, D. P. Wilkinson and J. Zhang, Chem. Rev., 2011, 111, 7625-7651.

22. D. Cazorla-Amoros, Front. Mater., 2014, 1, 1-3.

23. A. L. M. Reddy, S. R. Gowda, M. M. Shaijumon and P. M. Ajayan, Adv. Mater., 2012, 24, 50455064. 
24. T. Ben, C. Pei, D. Zhang, J. Xu, F. Deng, X. Jing and S. Qiu, Energy Environ. Sci., 2011, 4, 3991 3999.

25. H. Wang, Q. Gao and J. Hu, J. Am. Chem. Soc., 2009, 131, 7016-7022.

26. X. Yan, Y. Jia, L. Zhuang, L. Zhang, K. Wang and X. Yao, ChemElectroChem, 2018, 5, 18741879.

27. Y. Zhu, S. Murali, M. D. Stoller, K. J. Ganesh, W. Cai, P. J. Ferreira, A. Pirkle, R. M. Wallace, K. A. Cychosz, M. Thommes, D. Su, E. A. Stach and R. S. Ruoff, Science, 2011, 332, 1537-1541.

28. L. Fan, P. F. Liu, X. Yan, L. Gu, Z. Z. Yang, H. G. Yang, S. Qiu and X. Yao, Nat. Commun., 2016, 7, 10667 .

29. Y. Huang, J. Liang and Y. Chen, Small, 2012, 8, 1805-1834.

30. L. Dai, Y. Xue, L. Qu, H.-J. Choi and J.-B. Baek, Chem. Rev., 2015, 115, 4823-4892.

31. C. Zhu, H. Li, S. Fu, D. Du and Y. Lin, Chem. Soc. Rev., 2016, 45, 517-531.

32. X. Zhou, J. Qiao, L. Yang and J. Zhang, Adv. Energy Mater., 2014, 4, 1301523.

33. H. Zhang, H. Osgood, X. Xie, Y. Shao and G. Wu, Nano Energy, 2017, 31, 331-350.

34. S. Fu, C. Zhu, J. Song, D. Du and Y. Lin, Adv. Energy Mater., 2017, 7, 1700363.

35. H. M. Barkholtz and D.-J. Liu, Mater. Horiz., 2017, 4, 20-37.

36. Y. Jia, L. Zhang, A. Du, G. Gao, J. Chen, X. Yan, C. L. Brown and X. Yao, Adv. Mater., 2016, 28, 9532-9538.

37. H. Xu, D. Cheng, D. Cao and X. C. Zeng, Nat. Catal., 2018, 1, 339-348.

38. L. Zhang, Y. Jia, G. Gao, X. Yan, N. Chen, J. Chen, M. T. Soo, B. Wood, D. Yang, A. Du and X. Yao, Chem, 2018, 4, 285-297.

39. H. Fei, J. Dong, M. J. Arellano-Jimenez, G. Ye, N. Dong Kim, E. L. G. Samuel, Z. Peng, Z. Zhu, F. Qin, J. Bao, M. J. Yacaman, P. M. Ajayan, D. Chen and J. M. Tour, Nat. Commun., 2015, 6, 8668 .

40. P. Yin, T. Yao, Y. Wu, L. Zheng, Y. Lin, W. Liu, H. Ju, J. Zhu, X. Hong, Z. Deng, G. Zhou, S. Wei and Y. Li, Angew. Chem. Int. Ed., 2016, 55, 10800-10805.

41. C. Meng, T. Ling, T.-Y. Ma, H. Wang, Z. Hu, Y. Zhou, J. Mao, X.-W. Du, M. Jaroniec and S.-Z. Qiao, Adv. Mater., 2017, 29, 1604607.

42. J.-C. Liu, Y.-G. Wang and J. Li, J. Am. Chem. Soc., 2017, 139, 6190-6199.

43. A. Bruix, Y. Lykhach, I. Matolínová, A. Neitzel, T. Skála, N. Tsud, M. Vorokhta, V. Stetsovych, K. Ševčíková, J. Mysliveček, R. Fiala, M. Václavů, K. C. Prince, S. Bruyère, V. Potin, F. Illas, V. Matolín, J. Libuda and K. M. Neyman, Angew. Chem. Int. Ed., 2014, 53, 10525-10530.

44. H. Zhang, P. An, W. Zhou, B. Y. Guan, P. Zhang, J. Dong and X. W. Lou, Sci. Adv., 2018, 4.

45. P. Liu, R. Qin, G. Fu and N. Zheng, J. Am. Chem. Soc., 2017, 139, 2122-2131. 
46. X. Yu, M. R. Starke, L. M. Tolbert and B. Ozpineci, IET Electr. Power Appl., 2007, 1, 643-656.

47. M. W. Ellis, M. R. von Spakovsky and D. J. Nelson, Proc. - IEEE, 2001, 89, 1808-1818.

48. J.-H. Wee, Renew. Sust. Energ. Rev., 2007, 11, 1720-1738.

49. G. Liu, X. Li, P. Ganesan and B. N. Popov, Appl. Catal. B: Environ., 2009, 93, 156-165.

50. A. Guha, W. Lu, T. A. Zawodzinski Jr and D. A. Schiraldi, Carbon, 2007, 45, 1506-1517.

51. Y. Liang, Y. Li, H. Wang and H. Dai, J. Am. Chem. Soc., 2013, 135, 2013-2036.

52. X. Lin, L. Zheng, G. Gao, Y. Chi and G. Chen, Anal. Chem., 2012, 84, 7700-7707.

53. Y. H. Bing, H. S. Liu, L. Zhang, D. Ghosh and J. J. Zhang, Chem. Soc. Rev., 2010, 39, 2184-2202.

54. Z. Chen, D. Higgins, A. Yu, L. Zhang and J. Zhang, Energy Environ. Sci., 2011, 4, 3167-3192.

55. B. Narayanamoorthy, K. K. R. Datta, M. Eswaramoorthy and S. Balaji, ACS Appl. Mater. Interfaces, 2012, 4, 3620-3626.

56. Y. Zheng, Y. Jiao, M. Jaroniec, Y. Jin and S. Z. Qiao, Small, 2012, 8, 3550-3566.

57. R. Liu, D. Wu, X. Feng and K. Müllen, Angew. Chem. Int. Ed., 2010, 49, 2565-2569.

58. L. Qu, Y. Liu, J.-B. Baek and L. Dai, ACS Nano, 2010, 4, 1321-1326.

59. D. Geng, Y. Chen, Y. Chen, Y. Li, R. Li, X. Sun, S. Ye and S. Knights, Energy Environ. Sci., 2011, 4, 760-764.

60. S. Ni, Z. Li and J. Yang, Nanoscale, 2012, 4, 1184-1189.

61. H. Zhao, C. Sun, Z. Jin, D.-W. Wang, X. Yan, Z. Chen, G. Zhu and X. Yao, J. Mater. Chem. A, $2015,3,11736-11739$.

62. X. Zhao, X. Zou, X. Yan, C. L. Brown, Z. Chen, G. Zhu and X. Yao, Inorg. Chem. Front., 2016, 3, 417-421.

63. X. Yan, Y. Jia, T. Odedairo, X. Zhao, Z. Jin, Z. Zhu and X. Yao, Chem. Commun., 2016, 52, 81568159.

64. L. Zhang, Q. Xu, J. Niu and Z. Xia, Phys. Chem. Chem. Phys., 2015, 17, 16733-16743.

65. Y. Jiang, L. Yang, T. Sun, J. Zhao, Z. Lyu, O. Zhuo, X. Wang, Q. Wu, J. Ma and Z. Hu, ACS Catal., 2015, 5, 6707-6712.

66. C. Tang, H.-F. Wang, X. Chen, B.-Q. Li, T.-Z. Hou, B. Zhang, Q. Zhang, M.-M. Titirici and F. Wei, Adv. Mater., 2016, 28, 6845-6851.

67. Y. Jia, J. Chen and X. Yao, Mater. Chem. Front., 2018, 2, 1250-1268.

68. H. Chen, F. Sun, J. Wang, W. Li, W. Qiao, L. Ling and D. Long, J. Phys. Chem. C, 2013, 117, 8318-8328.

69. W. Shen and W. Fan, J. Mater. Chem. A, 2013, 1, 999-1013. 
70. Y. Xia and R. Mokaya, Adv. Mater., 2004, 16, 1553-1558.

71. J. Masa, W. Xia, M. Muhler and W. Schuhmann, Angew. Chem. Int. Ed., 2015, 54, 10102-10120.

72. S. Chen, J. Bi, Y. Zhao, L. Yang, C. Zhang, Y. Ma, Q. Wu, X. Wang and Z. Hu, Adv. Mater., 2012, 24, 5593-5597.

73. P. H. Matter, L. Zhang and U. S. Ozkan, J. Catal., 2006, 239, 83-96.

74. K. A. Kurak and A. B. Anderson, J. Phys. Chem. C, 2009, 113, 6730-6734.

75. L. Lai, J. R. Potts, D. Zhan, L. Wang, C. K. Poh, C. Tang, H. Gong, Z. Shen, J. Lin and R. S. Ruoff, Energy Environ. Sci., 2012, 5, 7936-7942.

76. L. J. Yang, S. J. Jiang, Y. Zhao, L. Zhu, S. Chen, X. Z. Wang, Q. Wu, J. Ma, Y. W. Ma and Z. Hu, Angew. Chem. Int. Ed., 2011, 50, 7132-7135.

77. D.-W. Wang and D. Su, Energy Environ. Sci., 2014, 7, 576-591.

78. S. Iijima, Nature, 1991, 354, 56-58.

79. T. Fu, R. Liu, J. Lv and Z. Li, Fuel Process. Technol., 2014, 122, 49-57.

80. W. Cui, Q. Liu, N. Cheng, A. M. Asiri and X. Sun, Chem. Commun., 2014, 50, 9340-9342.

81. W. Y. Wong, W. R. W. Daud, A. B. Mohamad, A. A. H. Kadhum, K. S. Loh and E. H. Majlan, Int. J. Hydrogen Energy, 2013, 38, 9421-9430.

82. J. Y. Lee, K. H. An, J. K. Heo and Y. H. Lee, J. Phys. Chem. B, 2003, 107, 8812-8815.

83. A. Q. Zhao, J. Masa, W. Schuhmann and W. Xia, J. Phys. Chem. C, 2013, 117, 24283-24291.

84. A. Siriviriyanun, T. Imae and N. Nagatani, Anal. Biochem., 2013, 443, 169-171.

85. W. Xiong, F. Du, Y. Liu, A. Perez, M. Supp, T. S. Ramakrishnan, L. M. Dai and L. Jiang, J. Am. Chem. Soc., 2010, 132, 15839-15841.

86. D. Yu, Q. Zhang and L. Dai, J. Am. Chem. Soc., 2010, 132, 15127-15129.

87. S. Y. Wang, E. Iyyamperumal, A. Roy, Y. H. Xue, D. S. Yu and L. M. Dai, Angew. Chem. Int. Ed., 2011, 50, 11756-11760.

88. S. Wang, D. Yu and L. Dai, J. Am. Chem. Soc., 2011, 133, 5182-5185.

89. D. Yu, Y. Xue and L. Dai, J. Phys. Chem. Lett., 2012, 3, 2863-2870.

90. G.-J. Sohn, H.-J. Choi, I.-Y. Jeon, D. W. Chang, L. Dai and J.-B. Baek, ACS Nano, 2012, 6, 63456355.

91. P. Zhang, J. S. Lian and Q. Jiang, Phys. Chem. Chem. Phys., 2012, 14, 11715-11723.

92. S. Kundu, T. C. Nagaiah, W. Xia, Y. M. Wang, S. Van Dommele, J. H. Bitter, M. Santa, G. Grundmeier, M. Bron, W. Schuhmann and M. Muhler, J. Phys. Chem. C, 2009, 113, 14302-14310.

93. Z. Chen, D. Higgins and Z. W. Chen, Carbon, 2010, 48, 3057-3065. 
94. Y. W. Ma, L. Y. Sun, W. Huang, L. R. Zhang, J. Zhao, Q. L. Fan and W. Huang, J. Phys. Chem. C, 2011, 115, 24592-24597.

95. Y. Li, J. Wang, X. Li, J. Liu, D. Geng, J. Yang, R. Li and X. Sun, Electrochem. Commun., 2011, 13, 668-672.

96. Z. Wang, R. Jia, J. Zheng, J. Zhao, L. Li, J. Song and Z. Zhu, ACS Nano, 2011, 5, 1677-1684.

97. M. Kaukonen, R. Kujala and E. Kauppinen, J. Phys. Chem. C, 2012, 116, 632-636.

98. R. M. Yadav, J. Wu, R. Kochandra, L. Ma, C. S. Tiwary, L. Ge, G. Ye, R. Vajtai, J. Lou and P. M. Ajayan, ACS Appl. Mater. Interfaces, 2015, 7, 11991-12000.

99. C. V. Rao, C. R. Cabrera and Y. Ishikawa, J. Phys. Chem. Lett., 2010, 1, 2622-2627.

100. A. Morozan, P. Jegou, M. Pinault, S. Campidelli, B. Jousselme and S. Palacin, ChemSusChem, $2012,5,647-651$.

101. T. Sharifi, G. Hu, X. Jia and T. Wågberg, ACS Nano, 2012, 6, 8904-8912.

102. L. R. Radovic, M. Karra, K. Skokova and P. A. Thrower, Carbon, 1998, 36, 1841-1854.

103. D. H. Zhong, H. Sano, Y. Uchiyama and K. Kobayashi, Carbon, 2000, 38, 1199-1206.

104. Y. Zhao, L. J. Yang, S. Chen, X. Z. Wang, Y. W. Ma, Q. Wu, Y. F. Jiang, W. J. Qian and Z. Hu, J. Am. Chem. Soc., 2013, 135, 1201-1204.

105. H. Khalfoun, P. Hermet, L. Henrard and S. Latil, Phys. Rev. B, 2010, 81, 193411.

106. D. V. Kosynkin, A. L. Higginbotham, A. Sinitskii, J. R. Lomeda, A. Dimiev, B. K. Price and J. M. Tour, Nature, 2009, 458, 872-876.

107. A. L. Higginbotham, D. V. Kosynkin, A. Sinitskii, Z. Sun and J. M. Tour, ACS Nano, 2010, 4, 2059-2069.

108. Y. Li, W. Zhou, H. Wang, L. Xie, Y. Liang, F. Wei, J.-C. Idrobo, S. J. Pennycook and H. Dai, Nat. Nanotechnol., 2012, 7, 394-400.

109. A. K. Geim and K. S. Novoselov, Nat. Mater., 2007, 6, 183-191.

110. A. K. Geim, Science, 2009, 324, 1530-1534.

111. M. J. Allen, V. C. Tung and R. B. Kaner, Chem. Rev., 2010, 110, 132-145.

112. L. Zhou, C. Zhang, X. Cai, Y. Qian, H. Jiang, B. Li, L. Lai, Z. Shen and W. Huang, ChemElectroChem, 2018, 5, 1811-1816.

113. X. Yan, Y. A. Jia, L. Zhang, M. T. Soo and X. Yao, Chem. Commun., 2017, 53, 12140-12143.

114. M. Qiao, C. Tang, G. He, K. Qiu, R. Binions, I. P. Parkin, Q. Zhang, Z. Guo and M. M. Titirici, J. Mater. Chem. A, 2016, 4, 12658-12666.

115. T. Odedairo, X. Yan, G. Gao, X. Yao, A. Du and Z. Zhu, Carbon, 2016, 107, 739-746.

116. S. Yasuda, L. Yu, J. Kim and K. Murakoshi, Chem. Commun., 2013, 49, 9627-9629. 
117. X. Wang, G. Sun, P. Routh, D.-H. Kim, W. Huang and P. Chen, Chem. Soc. Rev., 2014, 43, $7067-$ 7098 .

118. B. Zheng, P. Hermet and L. Henrard, ACS Nano, 2010, 4, 4165-4173.

119. Y. Tison, J. Lagoute, V. Repain, C. Chacon, Y. Girard, S. Rousset, F. Joucken, D. Sharma, L. Henrard, H. Amara, A. Ghedjatti and F. Ducastelle, ACS Nano, 2015, 9, 670-678.

120. Z. Hou, X. Wang, T. Ikeda, K. Terakura, M. Oshima, M.-a. Kakimoto and S. Miyata, Phys. Rev. B, $2012,85,165439$.

121. L. P. Zhang and Z. H. Xia, J. Phys. Chem. C, 2011, 115, 11170-11176.

122. D. Deng, X. Pan, L. Yu, Y. Cui, Y. Jiang, J. Qi, W.-X. Li, Q. Fu, X. Ma, Q. Xue, G. Sun and X. Bao, Chem. Mater., 2011, 23, 1188-1193.

123. V. W. Brar, Y. Zhang, Y. Yayon, T. Ohta, J. L. McChesney, A. Bostwick, E. Rotenberg, K. Horn and M. F. Crommie, Appl. Phys. Lett., 2007, 91, 122102.

124. P. Lauffer, K. V. Emtsev, R. Graupner, T. Seyller, L. Ley, S. A. Reshanov and H. B. Weber, Phys. Rev. B, 2008, 77, 155426.

125. D. Wei, Y. Liu, Y. Wang, H. Zhang, L. Huang and G. Yu, Nano Lett., 2009, 9, 1752-1758.

126. A. Zabet-Khosousi, L. Zhao, L. Pálová, M. S. Hybertsen, D. R. Reichman, A. N. Pasupathy and G. W. Flynn, J. Am. Chem. Soc., 2014, 136, 1391-1397.

127. Y. Wang, Y. Shao, D. W. Matson, J. Li and Y. Lin, ACS Nano, 2010, 4, 1790-1798.

128. B. Guo, Q. Liu, E. Chen, H. Zhu, L. Fang and J. R. Gong, Nano Lett., 2010, 10, 4975-4980.

129. X. Li, H. Wang, J. T. Robinson, H. Sanchez, G. Diankov and H. Dai, J. Am. Chem. Soc., 2009, 131, 15939-15944.

130. Z.-H. Sheng, L. Shao, J.-J. Chen, W.-J. Bao, F.-B. Wang and X.-H. Xia, ACS Nano, 2011, 5, 43504358.

131. Z. Y. Lin, M. K. Song, Y. Ding, Y. Liu, M. L. Liu and C. P. Wong, Phys. Chem. Chem. Phys., 2012, 14, 3381-3387.

132. Z. Y. Lin, G. Waller, Y. Liu, M. L. Liu and C. P. Wong, Adv. Energy Mater., 2012, 2, 884-888.

133. S. Yang, L. Zhi, K. Tang, X. Feng, J. Maier and K. Müllen, Adv. Funct. Mater., 2012, 22, 36343640.

134. J. Wang, R. Ma, Z. Zhou, G. Liu and Q. Liu, Sci. Rep., 2015, 5, 9304.

135. Z. Yang, Z. Yao, G. Li, G. Fang, H. Nie, Z. Liu, X. Zhou, X. a. Chen and S. Huang, ACS Nano, $2011,6,205-211$.

136. I. Y. Jeon, S. Zhang, L. P. Zhang, H. J. Choi, J. M. Seo, Z. H. Xia, L. M. Dai and J. B. Baek, Adv. Mater., 2013, 25, 6138-6145.

137. L. P. Zhang, J. B. Niu, M. T. Li and Z. H. Xia, J. Phys. Chem. C, 2014, 118, 3545-3553. 
138. I.-Y. Jeon, H.-J. Choi, M. Choi, J.-M. Seo, S.-M. Jung, M.-J. Kim, S. Zhang, L. Zhang, Z. Xia, L. Dai, N. Park and J.-B. Baek, Sci. Rep., 2013, 3, 1810.

139. Z. Yao, H. Nie, Z. Yang, X. Zhou, Z. Liu and S. Huang, Chem. Commun., 2012, 48, 1027-1029.

140. S. Jiang, Y. Sun, H. Dai, J. Hu, P. Ni, Y. Wang, Z. Li and Z. Li, Nanoscale, 2015, 7, 10584-10589.

141. J. Zhang and L. Dai, Angew. Chem. Int. Ed., 2016, 55, 13296-13300.

142. X. J. Sun, Y. W. Zhang, P. Song, J. Pan, L. Zhuang, W. L. Xu and W. Xing, ACS Catal., 2013, 3, 1726-1729.

143. X. J. Sun, P. Song, T. Chen, J. Liu and W. L. Xu, Chem. Commun., 2013, 49, 10296-10298.

144. S. Lee, M. Choun, Y. Ye, J. Lee, Y. Mun, E. Kang, J. Hwang, Y. H. Lee, C. H. Shin, S. H. Moon, S. K. Kim and E. Lee, Angew. Chem. Int. Ed., 2015, 54, 9230-9234.

145. W. Niu, L. Li, X. Liu, N. Wang, J. Liu, W. Zhou, Z. Tang and S. Chen, J. Am. Chem. Soc., 2015, 137, 5555-5562.

146. S. B. Yoon, J. Y. Kim, F. Kooli, C. W. Lee and J.-S. Yu, Chem. Commun., 2003, 0, 1740-1741.

147. S. B. Yoon, G. S. Chai, S. K. Kang, J.-S. Yu, K. P. Gierszal and M. Jaroniec, J. Am. Chem. Soc., 2005, 127, 4188-4189.

148. X. Ji, K. T. Lee and L. F. Nazar, Nat. Mater., 2009, 8, 500-506.

149. T.-W. Kim, H.-D. Kim, K.-E. Jeong, H.-J. Chae, S.-Y. Jeong, C.-H. Lee and C.-U. Kim, Green Chem., 2011, 13, 1718-1728.

150. H. Chang, S. H. Joo and C. Pak, J. Mater. Chem., 2007, 17, 3078-3088.

151. T. Yang, J. Liu, R. Zhou, Z. Chen, H. Xu, S. Z. Qiao and M. J. Monteiro, J. Mater. Chem. A, 2014, 2, 18139-18146.

152. B. Bayatsarmadi, Y. Zheng, M. Jaroniec and S. Z. Qiao, Chem. Asian J., 2015, 10, 1546-1553.

153. J. Yan, H. Meng, F. Y. Xie, X. L. Yuan, W. D. Yu, W. R. Lin, W. P. Ouyang and D. S. Yuan, J. Power Sources, 2014, 245, 772-778.

154. W. Wei, H. W. Liang, K. Parvez, X. D. Zhuang, X. L. Feng and K. Mullen, Angew. Chem. Int. Ed., 2014, 53, 1570-1574.

155. H.-P. Cong, P. Wang, M. Gong and S.-H. Yu, Nano Energy, 2014, 3, 55-63.

156. K. Kwon, Y. J. Sa, J. Y. Cheon and S. H. Joo, Langmuir, 2012, 28, 991-996.

157. X. Bo and L. Guo, Phys. Chem. Chem. Phys., 2013, 15, 2459-2465.

158. D.-S. Yang, D. Bhattacharjya, S. Inamdar, J. Park and J.-S. Yu, J. Am. Chem. Soc., 2012, 134, $16127-16130$.

159. S. Zhang, A. Ikoma, K. Ueno, Z. Chen, K. Dokko and M. Watanabe, ChemSusChem, 2015, 8, 1608-1617.

160. J. Liang, Y. Jiao, M. Jaroniec and S. Z. Qiao, Angew. Chem. Int. Ed., 2012, 51, 11496-11500. 
161. R. Silva, D. Voiry, M. Chhowalla and T. Asefa, J. Am. Chem. Soc., 2013, 135, 7823-7826.

162. Z. Liu, F. Peng, H. Wang, H. Yu, J. Tan and L. Zhu, Catal. Commun., 2011, 16, 35-38.

163. Z.-W. Liu, F. Peng, H.-J. Wang, H. Yu, W.-X. Zheng and J. Yang, Angew. Chem., 2011, 123, 33153319.

164. P. Pachfule, V. M. Dhavale, S. Kandambeth, S. Kurungot and R. Banerjee, Chem. Eur. J., 2013, 19, 974-980.

165. Z. Y. Mo, R. P. Zheng, H. L. Peng, H. G. Liang and S. J. Liao, J. Power Sources, 2014, 245, 801807.

166. D. U. Lee, H. W. Park, D. Higgins, L. Nazar and Z. Chen, J. Electrochem. Soc., 2013, 160, F910F915.

167. C. Zhu, J. Zhai and S. Dong, Chem. Commun., 2012, 48, 9367-9369.

168. M. Park, T. Lee and B.-S. Kim, Nanoscale, 2013, 5, 12255-12260.

169. H. Kim, K. Lee, S. I. Woo and Y. Jung, Phys. Chem. Chem. Phys., 2011, 13, 17505-17510.

170. B. Sanyal, O. Eriksson, U. Jansson and H. Grennberg, Phys. Rev. B, 2009, 79, 113409.

171. F. Banhart, J. Kotakoski and A. V. Krasheninnikov, ACS Nano, 2010, 5, $26-41$.

172.J. Kotakoski, A. V. Krasheninnikov, U. Kaiser and J. C. Meyer, Phys. Rev. Lett., 2011, 106, 105505.

173. S. Okada, T. Kawai and K. Nakada, J. Phys. Soc. Jpn., 2011, 80, 013709.

174. M. M. Ugeda, I. Brihuega, F. Hiebel, P. Mallet, J.-Y. Veuillen, J. M. Gómez-Rodríguez and F. Ynduráin, Phys. Rev. B, 2012, 85, 121402.

175. J. Zhao, H. Zeng, J. Wei, B. Li and D. Xu, Phys. Lett. A, 2014, 378, 416-420.

176. L. Liu, M. Qing, Y. Wang and S. Chen, J. Mater. Sci. Technol., 2015, 31, 599-606.

177. J. I. Feldblyum, A. G. Wong-Foy and A. J. Matzger, Chem. Commun., 2012, 48, 9828-9830.

178. L. Wang, A. Ambrosi and M. Pumera, Angew. Chem. Int. Ed., 2013, 52, 13818-13821.

179. J. Masa, A. Zhao, W. Xia, Z. Sun, B. Mei, M. Muhler and W. Schuhmann, Electrochem. Commun., 2013, 34, 113-116.

180. J. Liu, X. Sun, P. Song, Y. Zhang, W. Xing and W. Xu, Adv. Mater., 2013, 25, 6879-6883.

181. R. A. Sidik, A. B. Anderson, N. P. Subramanian, S. P. Kumaraguru and B. N. Popov, J. Phys. Chem. B, 2006, 110, 1787-1793.

182. L. Yu, X. L. Pan, X. M. Cao, P. Hu and X. H. Bao, J. Catal., 2011, 282, 183-190.

183. Y. Sha, T. H. Yu, Y. Liu, B. V. Merinov and W. A. Goddard, J. Phys. Chem. Lett., 2010, 1, 856861.

184. W. Wei, L. Haiwei, P. Khaled, Z. Xiaodong, F. Xinliang and M. Klaus, Angew. Chem. Int. Ed., 2014, 53, 1570-1574. 
185. L. Ji, Z. Yao, C. Jun, L. Jian, H.-J. Denisa, J. Mietek and Q. S. Zhang, Angew. Chem. Int. Ed., 2012, 51, 3892-3896.

186. C. Sheng, B. Jiyu, Z. Yu, Y. Lijun, Z. Chen, M. Yanwen, W. Qiang, W. Xizhang and H. Zheng, Adv. Mater., 2012, 24, 5593-5597.

187. Y. Jiao, Y. Zheng, M. Jaroniec and S. Z. Qiao, J. Am. Chem. Soc., 2014, 136, 4394-4403.

188. C. E. Banks, M. R. Moore, T. J. Davies and R. G. Compton, Chem. Commun., 2004, 10, 18041805.

189. C. E. Banks, T. J. Davies, G. G. Wildgoose and R. G. Compton, Chem. Commun., 2005, 0, 829841.

190. P. M. Hallam and C. E. Banks, Electrochem. Commun., 2011, 13, 8-11.

191. M. Pumera, Chem. Rec., 2012, 12, 201-213.

192. A. Shen, Y. Zou, Q. Wang, R. A. W. Dryfe, X. Huang, S. Dou, L. Dai and S. Wang, Angew. Chem. Int. Ed., 2014, 53, 10804-10808.

193. G. Y. Zhong, H. J. Wang, H. Yu and F. Peng, Electrochem. Commun., 2014, 40, 5-8.

194. X. Hu, Y. Wu, H. Li and Z. Zhang, J. Phys. Chem. C, 2010, 114, 9603-9607.

195. L. Tao, Q. Wang, S. Dou, Z. Ma, J. Huo, S. Wang and L. Dai, Chem. Commun., 2016, 52, 27642767.

196. D. W. Boukhvalov and M. I. Katsnelson, Nano Lett., 2008, 8, 4373-4379.

197. D. Lu, D. Wu, J. Jin and L. Chen, Electrochim. Acta, 2016, 215, 66-71.

198. Y. Tian, Y. Ye, X. Wang, S. Peng, Z. Wei, X. Zhang and W. Liu, Appl. Catal. A: Gen., 2017, 529, $127-133$.

199. Y. Tian, Z. Wei, X. Wang, S. Peng, X. Zhang and W. M. Liu, Int. J. Hydrogen Energy, 2017, 42, 4184-4192.

200. K. Chu, F. Wang, Y. Tian and Z. Wei, Electrochim. Acta, 2017, 231, 557-564.

201. C. Tang and Q. Zhang, Adv. Mater., 2017, 29, 1604103.

202. Z. Lu, J. Wang, S. Huang, Y. Hou, Y. Li, Y. Zhao, S. Mu, J. Zhang and Y. Zhao, Nano Energy, 2017, 42, 334-340.

203. J.-H. Zhong, J. Zhang, X. Jin, J.-Y. Liu, Q. Li, M.-H. Li, W. Cai, D.-Y. Wu, D. Zhan and B. Ren, J. Am. Chem. Soc., 2014, 136, 16609-16617.

204. W. Zhang and W. Zheng, Adv. Funct. Mater., 2016, 26, 2988-2993.

205. L. Liu and A. Corma, Chem. Rev., 2018, 118, 4981-5079.

206. X.-F. Yang, A. Wang, B. Qiao, J. Li, J. Liu and T. Zhang, Acc. Chem. Res., 2013, 46, 1740-1748.

207. B. Qiao, A. Wang, X. Yang, L. F. Allard, Z. Jiang, Y. Cui, J. Liu, J. Li and T. Zhang, Nat. Chem., $2011,3,634-641$. 
208. J. Lin, A. Wang, B. Qiao, X. Liu, X. Yang, X. Wang, J. Liang, J. Li, J. Liu and T. Zhang, J. Am. Chem. Soc., 2013, 135, 15314-15317.

209. P. Liu, Y. Zhao, R. Qin, S. Mo, G. Chen, L. Gu, D. M. Chevrier, P. Zhang, Q. Guo, D. Zang, B. Wu, G. Fu and N. Zheng, Science, 2016, 352, 797-800.

210. J. Liu, ACS Catal., 2017, 7, 34-59.

211. H. Wang, Q. Wang, Y. Cheng, K. Li, Y. Yao, Q. Zhang, C. Dong, P. Wang, U. Schwingenschlögl, W. Yang and X. X. Zhang, Nano Lett., 2012, 12, 141-144.

212. C. Yuanjun, J. Shufang, W. Yanggang, D. Juncai, C. Wenxing, L. Zhi, S. Rongan, Z. Lirong, Z. Zhongbin, W. Dingsheng and L. Yadong, Angew. Chem. Int. Ed., 2017, 56, 6937-6941.

213. J. Wang, Z. Huang, W. Liu, C. Chang, H. Tang, Z. Li, W. Chen, C. Jia, T. Yao, S. Wei, Y. Wu and Y. Li, J. Am. Chem. Soc., 2017, 139, 17281-17284.

214. C. Tang, H.-F. Wang and Q. Zhang, Acc. Chem. Res., 2018, 51, 881-889.

215. D. Yan, Y. Li, J. Huo, R. Chen, L. Dai and S. Wang, Adv. Mater., 2017, 29, 1606459.

216. Q. Wu, L. Yang, X. Wang and Z. Hu, Acc. Chem. Res., 2017, 50, 435-444.

217. A. Wang, J. Li and T. Zhang, Nat. Rev. Chem., 2018, 2, 65-81.

218. Y. Yang, X. Liu, Z. Zhu, Y. Zhong, Y. Bando, D. Golberg, J. Yao and X. Wang, Joule, 2018, 2, 1075-1094.

219. X. Yan, Y. Jia, J. Chen, Z. Zhu and X. Yao, Adv. Mater., 2016, 28, 8771-8778.

220. H. Fei, J. Dong, Y. Feng, C. S. Allen, C. Wan, B. Volosskiy, M. Li, Z. Zhao, Y. Wang, H. Sun, P. An, W. Chen, Z. Guo, C. Lee, D. Chen, I. Shakir, M. Liu, T. Hu, Y. Li, A. I. Kirkland, X. Duan and Y. Huang, Nat. Catal., 2018, 1, 63-72.

221. C. H. Choi, M. Kim, H. C. Kwon, S. J. Cho, S. Yun, H.-T. Kim, K. J. J. Mayrhofer, H. Kim and M. Choi, Nat. Commun., 2016, 7.

222. H. B. Yang, S.-F. Hung, S. Liu, K. Yuan, S. Miao, L. Zhang, X. Huang, H.-Y. Wang, W. Cai, R. Chen, J. Gao, X. Yang, W. Chen, Y. Huang, H. M. Chen, C. M. Li, T. Zhang and B. Liu, Nat. Energy, 2018, 3, 140-147.

223. K. S. Novoselov, A. Mishchenko, A. Carvalho and A. H. Castro Neto, Science, 2016, 353.

224. C. Tang, L. Zhong, B. Zhang, H.-F. Wang and Q. Zhang, Adv. Mater., 2018, 30, 1705110.

225. Y. Jia, L. Zhang, G. Gao, H. Chen, B. Wang, J. Zhou, M. T. Soo, M. Hong, X. Yan, G. Qian, J. Zou, A. Du and X. Yao, Adv. Mater., 2017, 29, 1700017.

226. N. Ma, Y. Jia, X. Yang, X. She, L. Zhang, Z. Peng, X. Yao and D. Yang, J. Mater. Chem. A, 2016, 4, 6376-6384.

227. L. Liu, X. Yang, N. Ma, H. Liu, Y. Xia, C. Chen, D. Yang and X. Yao, Small, 2016, 12, $1295-$ 1301.

228. D. Yang, L. Zhang, X. Yan and X. Yao, Small Methods, 2017, 1, 1700209. 